A One Parameter Family of
Expanding Wave Solutions
of the
Einstein Equations
that induces an
Anomalous Acceleration
into the
Standard Model of Cosmology
Blake Temple, UC-Davis
Collaborator: J. Smoller (PNAS: August 2009)




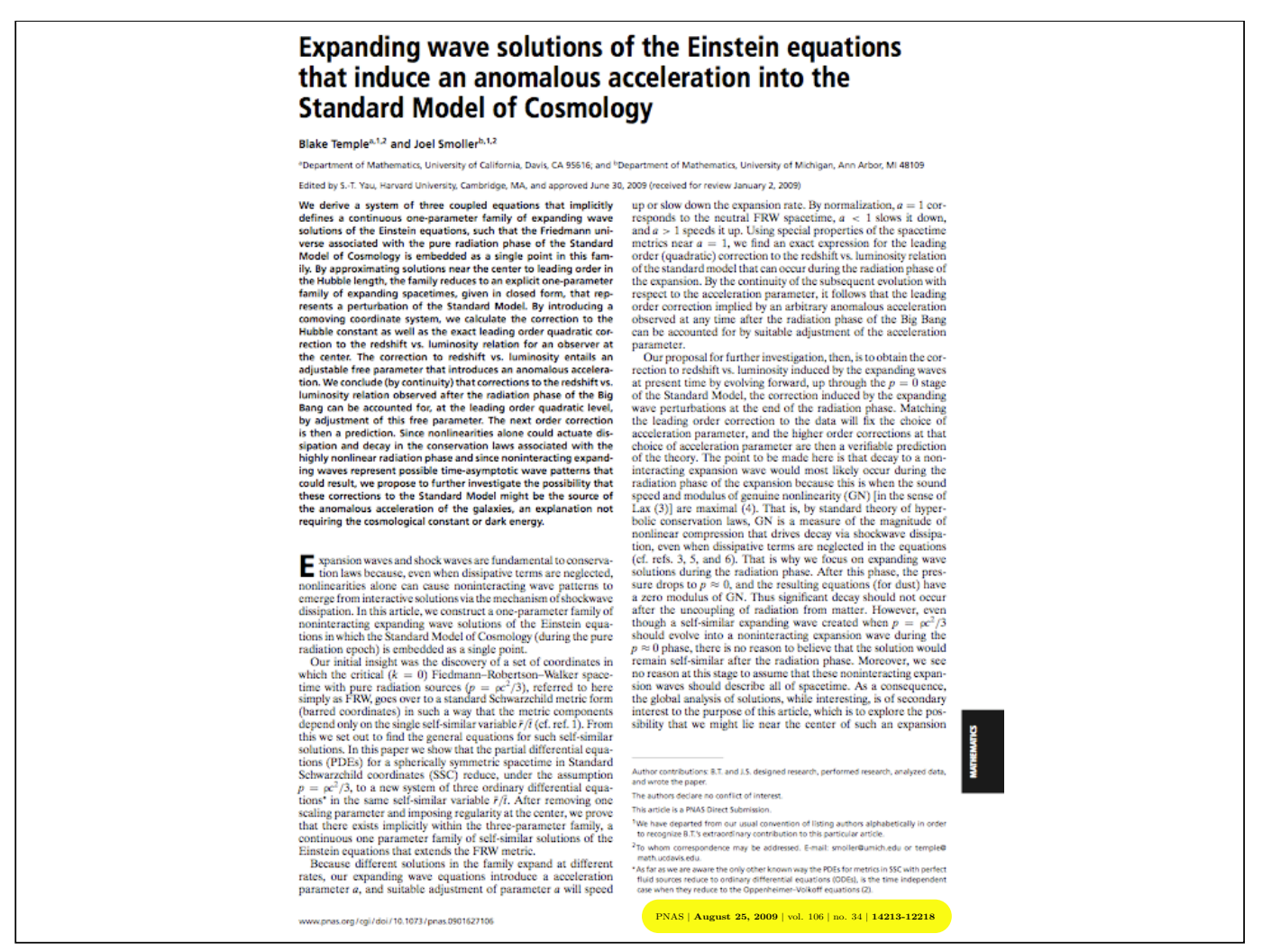


PNAS | August 25, 2009 | vol. 106 | no. 34 | 14213-12218 


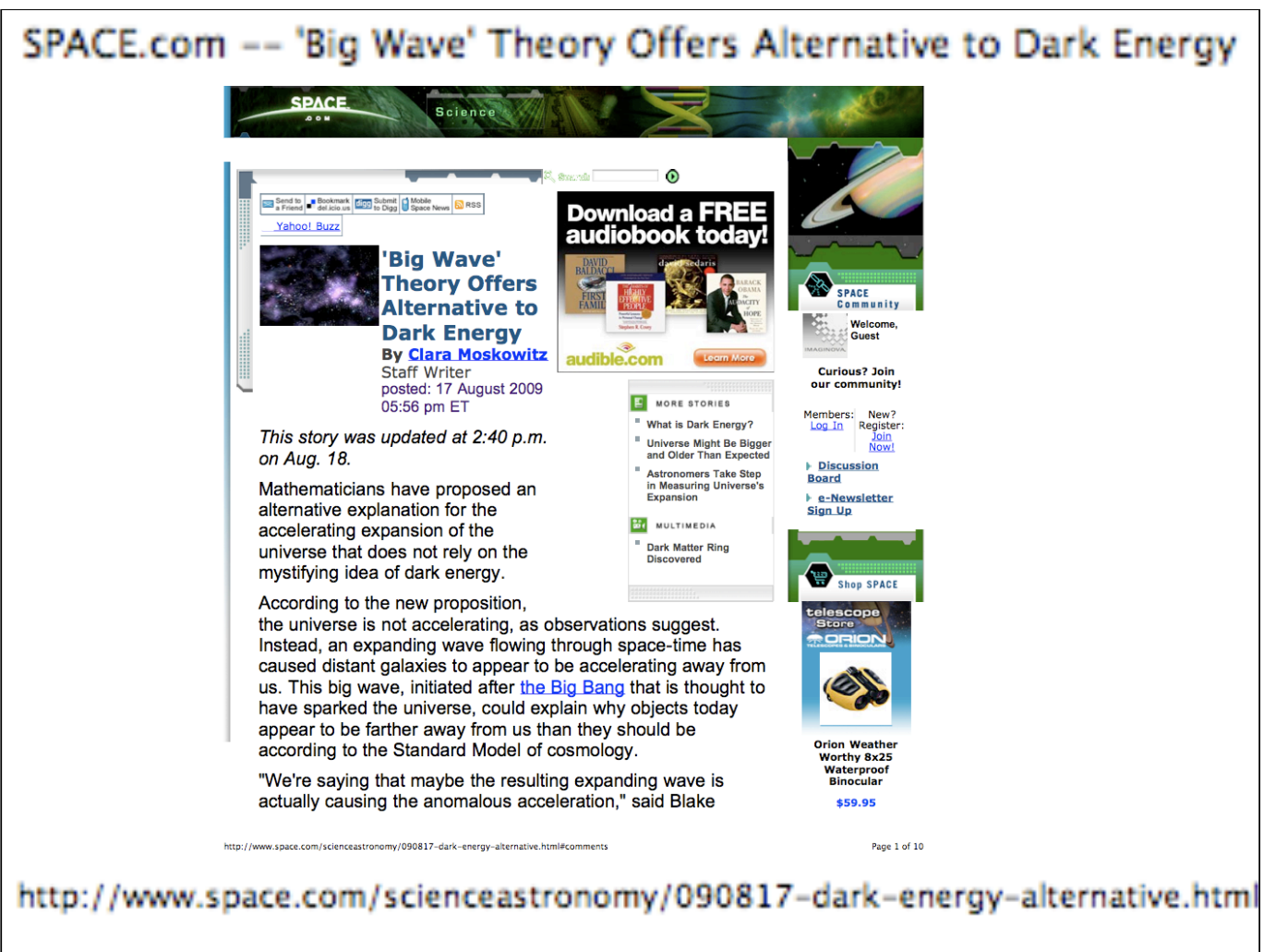


NATIONAL GEOGRAPHIC NEWS

NATIONALGEOGRAPHICCOM/NEWS

Dark Energy's Demise? New Theory Doesn't Use the Force

Ker Than
for National Geograenic News

August 18, 2009

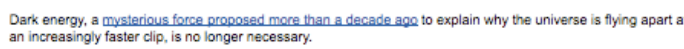

That's the onclusion of a controversial new theory hat shows how the accelerated expansion of the universe

In a new study, two mathematicians presener their solutions to Einstien's field equations of general relativity.
which describe the relationship between gravity and master.

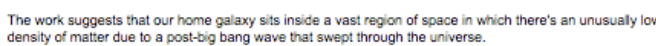

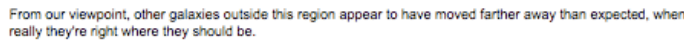

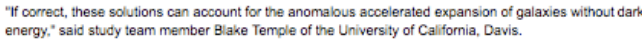

(n)

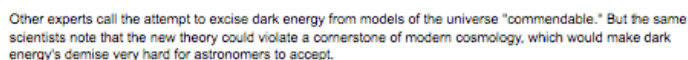

Dark Energy Alternative

Untb 1998 astronomers had thought that gravily should be slowing down the cosnic exxansion triggered by the
big bann.

That year two independent teams announced data showing that the universe's expansion is speeding up.

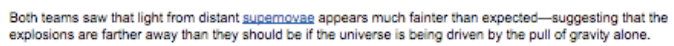

To explan his observation, astronomers started to entertain the idea of dark energy, a universal repusisve focce

Still, more than ten years later, no one is sure what dark energy is $-\infty$ if if treally exists

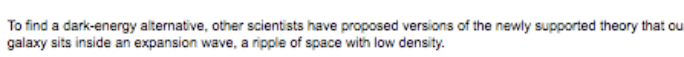


'Big Wwe' Theory Offers A:ternative to Dark Energy (SPACE com) - Dev Articles

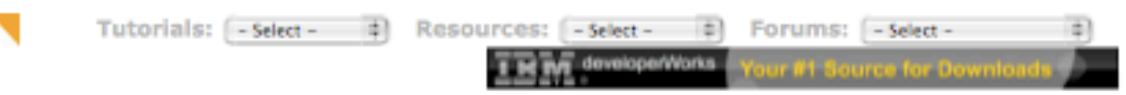

\section{Science News}

|Forums: \% Register " | User CP | Games | Calendar | Members | FAQs |
Sitemap | Support | [

User Name:

User Name

Password:

(Log in

$\checkmark$ Remember

me

- Dev Articles Community Forums > Latest News Updated Hourly > Science News

a 'Big Wave' Theory Offers Alternative to Dark Energy (SPACE.com)

Discuss 'Big Wave' Theory Offers Alternative to Dark Energy (SPACE.com) in the

Science News forum on Dev Articles.

Dark Eneroy (SPACE.com) Read about and discuss the

latest happenings in science.

Learn and Grow with IBMO AJAX + Microsoft .NET $=$

Tech News You Can't Use!

EASY

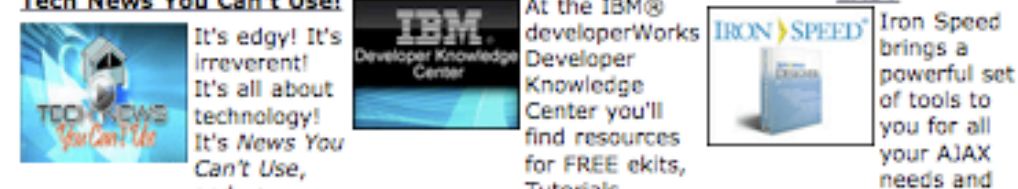




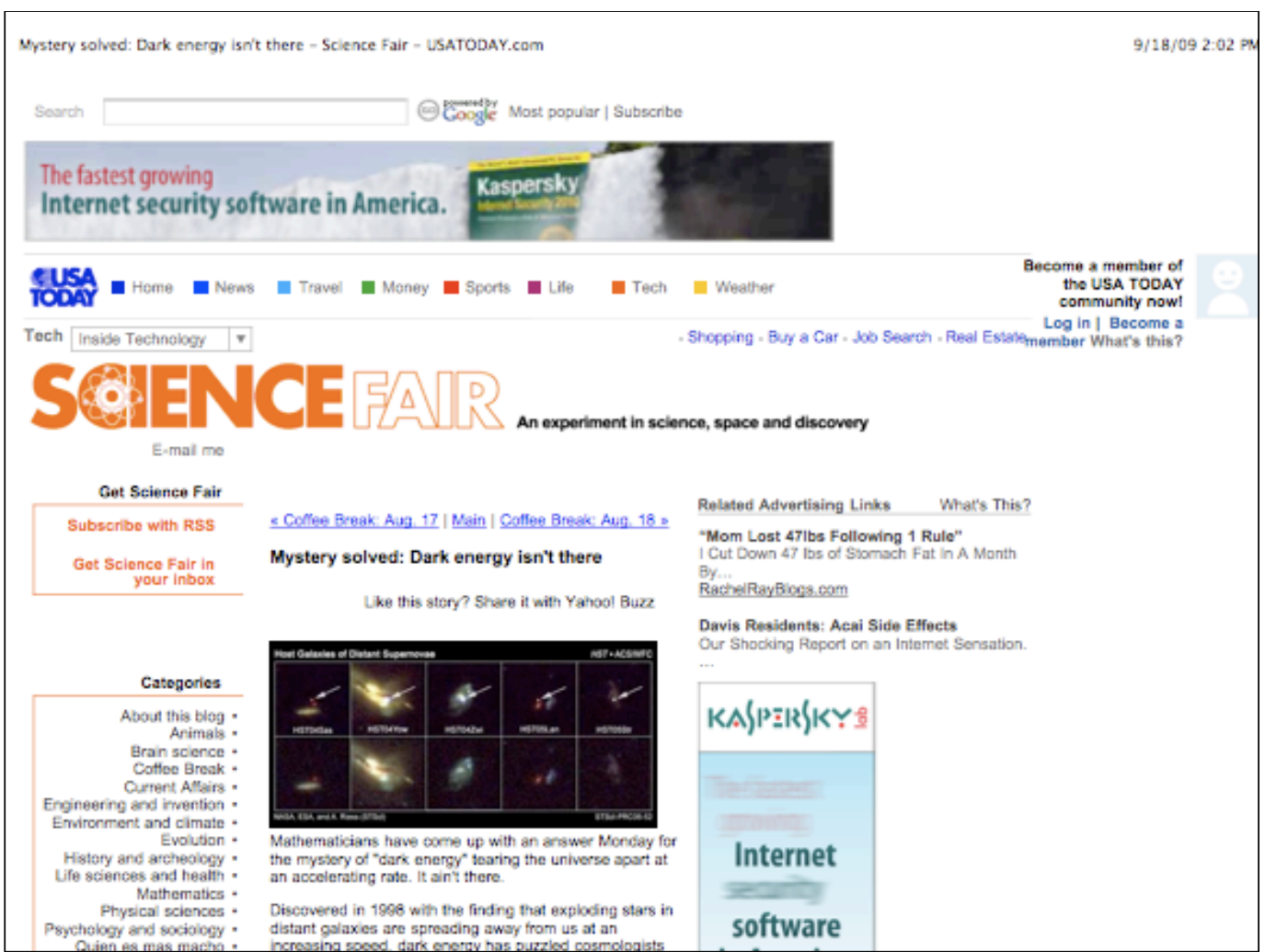


YAFOOI NEWS

US pair shed light on Dark Energy

Today, 05:36 a

PRESS

Two mathematicians have boldly tampered with Albert Einstein's equations to show that "Dark Energy", the mysterious anti-gravity force that

theoretically makes up three-quarters of the universe, might not exist after all.

Dark Energy is thought to be the reason why galaxies appear to be accelerating away from each other at increasing speed.

It acts as a kind of anti-gravity force which repels instead of attracts.

Einstein first came up with the idea as a modification to his theory of general relativity. The "cosmological constant", as he called it, was invoked to prevent the universe collapsing under he pull of gravity.

But Einstein was not happy with the concept, and abandoned it after astronomers discovered that the universe might be expanding rather than standing still.

Later it was confirmed that the universe was not only expanding, but accelerating outwards. To account for this acceleration, physicists resurrected the cosmological constant in the new guise of "Dark Energy".

The theory suggests that Dark Energy makes up nearly $75 \%$ of the interchangeable mass and energy in the universe.

Two US mathematicians, Professor Blake Temple from the University of California at Davis, and Dr Joel Smoller, from the University of Michigan in Ann Arbor, have now tweaked

They suggest that expanding waves of space-time could emerge from the initial disturbance

Professor Ofer Lahav, head of astrophysics at University College London, who is part of a

team investigating Dark Energy, said: "This is a thought-provoking paper, challenging the

astronomers found in many different ways that 'Dark Enery' is requied in ore. In a nutshel, data. In this interpretation, $75 \%$ of the universe at present is made of Dark Energy "

Buzz Up! Email Story Share Story Print Story
ADVERTISEMENT

Want to see my Experian credit

It's FREE

Tmiustarious to seeit

Iam applying ro my credit rating

lam applying for a mortgage

Im woried a tor a lD theth

Creditexpert

membership today

Print Story




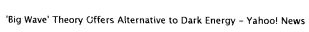

YAHOO! NEWS

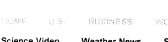

픙

'Big Wave' Theory Offers Alternative to Dark Energy SPRCE

$$
\text { Top Stories }
$$

This story was unoateded at 240 p.m.m. on Ang. 18.

$$
\text { aurat we }
$$

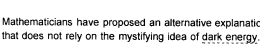

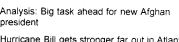

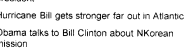

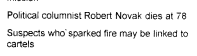

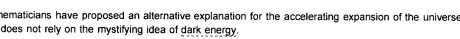

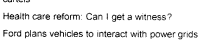

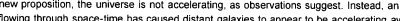

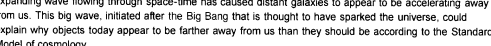

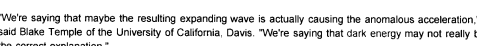

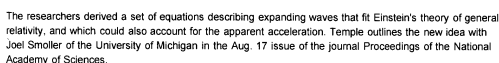

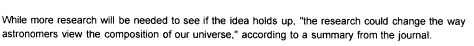

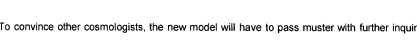

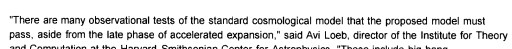

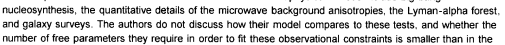

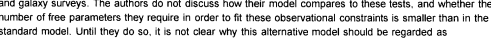

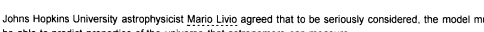

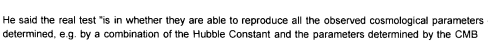




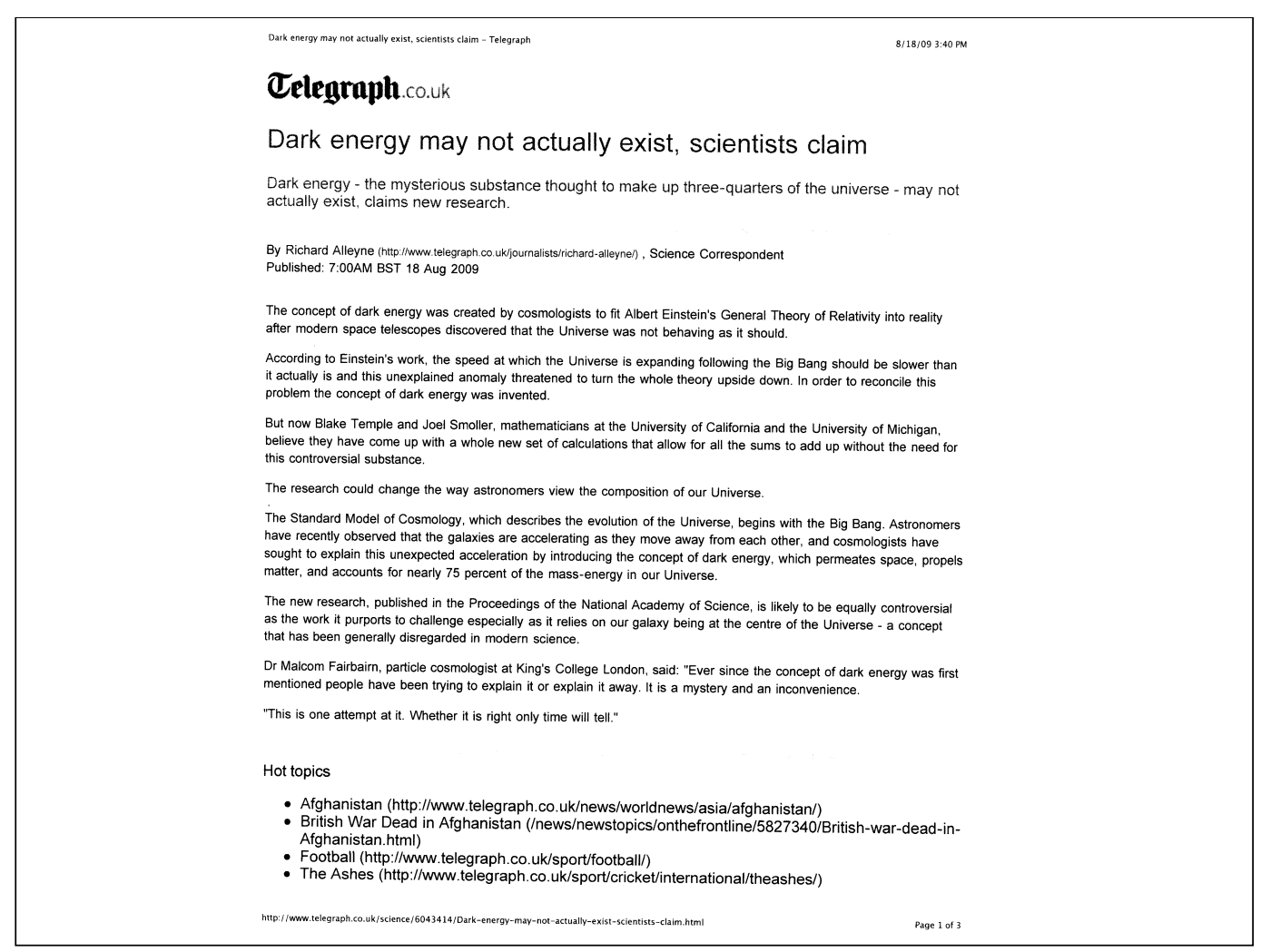




\section{Seed Magazineabout}

SEEDMAGAZINE.COM September 24, 2009 Subscribe to the magazine $»$ RSS \& Email Updates $»$ Follow us on Twitter

\section{Erasing Dark Energy}

Wide Angle / by Veronique Greenwood / September 24, 2009

Why do we need dark energy to explain the observable

universe? Two mathematicians propose an alternate solution

that, while beautiful, may raise even more questions than it

that, while

- ShareThis

- Print

Page 1 of 2

12 Next "

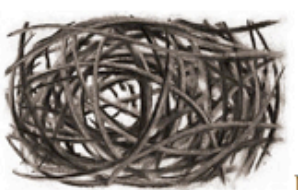

Illustration by Mike Pick

Against all reason, the universe is accelerating its expansion. 


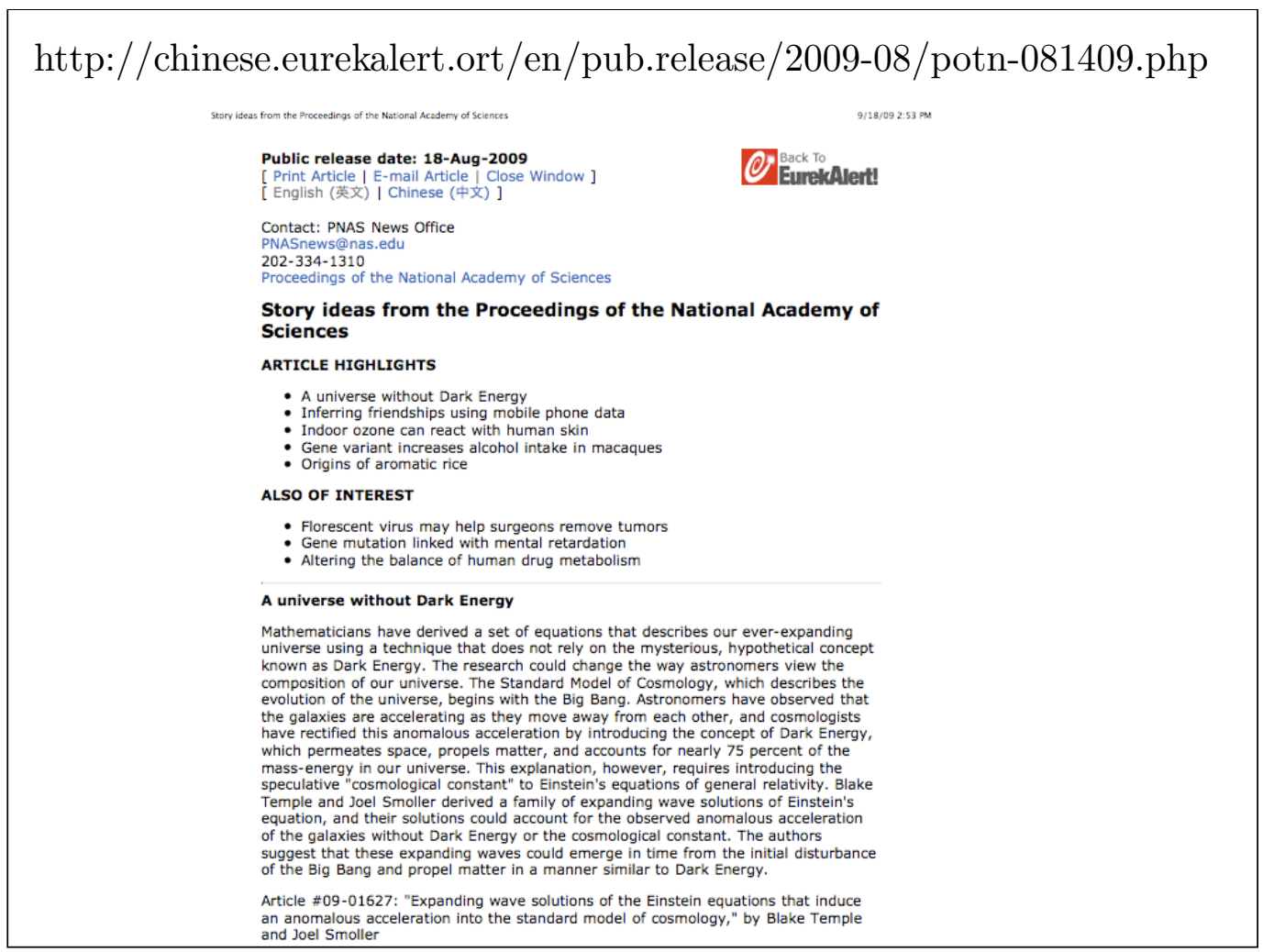




\section{UCDAVIS}

\section{egghead s}

A Big Wave after the Big Bang?

August 18th, $2009 @$ 2:06 pm by andy

Mathematicians Blake Temple from UC Davis and Joel Smoller from the University of Michigan have published a new theory to explain why the universe appears to be expanding at an accelerating pace without invoking "dark energy."

About a decade ago, astronomers realized that the universe is not only expanding - the expansion appears to be speeding up. To explain this, they came up with the concept of dark energy: a force that pushes the galaxies apart. No one knows what dark energy actually is; one idea is that is a sort of energy that bubbles out of the fabric of space as it expands. Physicists' calculations, though, show dark matter, which is nearly as mysterious: matter and energy that we can feel and touch make up trivial portion of the universe).

Temple and Smoller though, have a different explanation for why the galaxies are further apart than they ought to be. A "big wave," started after the Big Bang at the beginning of the universe, is

"We're saying that maybe the resulting expanding wave is actually causing the anomalous ce.com.

Several other cosmologists quoted by Space.com were sceptical, noting that the new theory needs to astronomers and physicists.

The paper is published in the Aug. 17 issue of the Proceedings of the National Academy of Sciences.

For an overview of physics, cosmology and dark energy, read this.

Share with social bookmarking:

- 国 


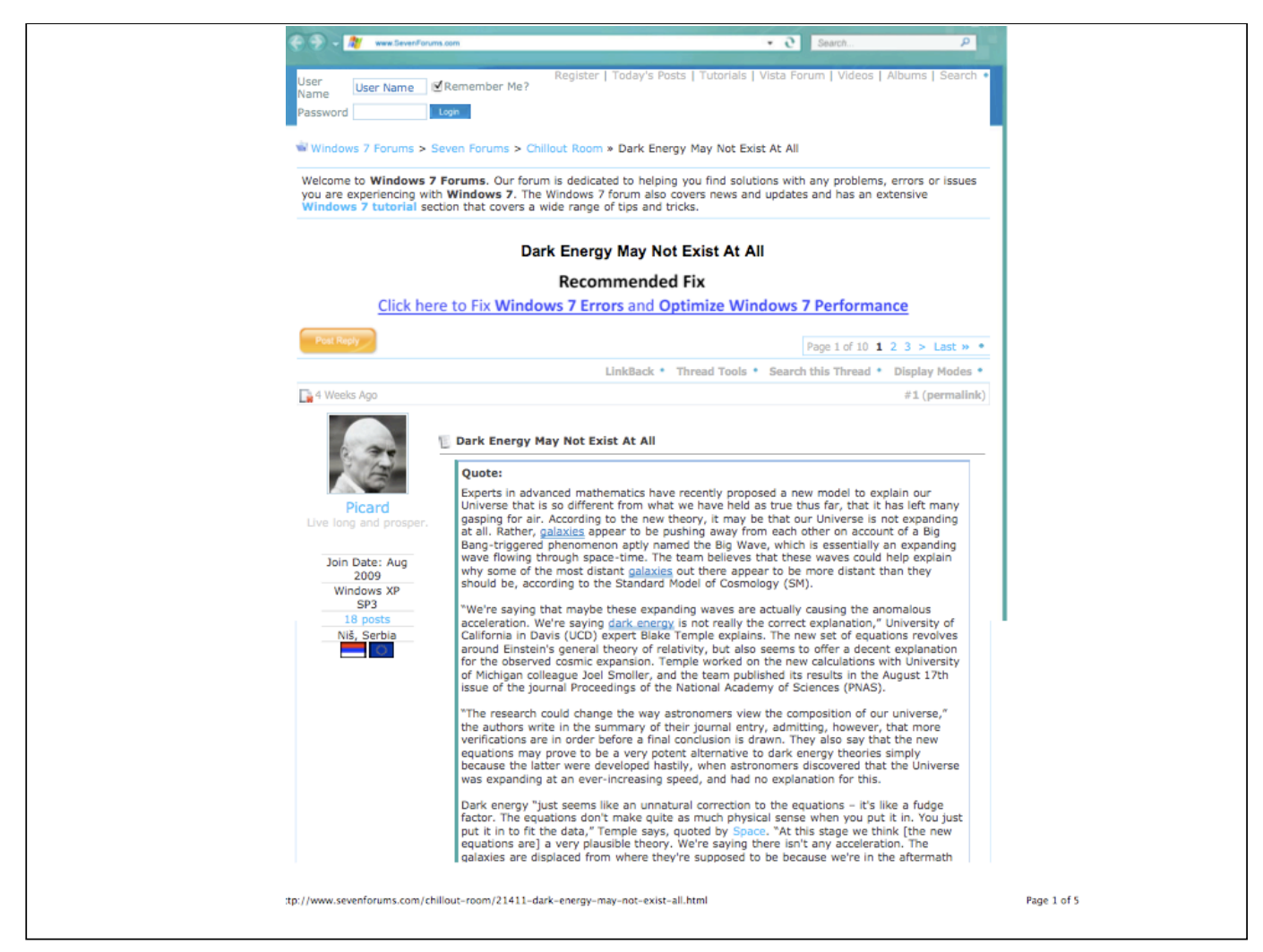




Quote:
Experts in advanced mathematics have recently proposed a new model to explain our
Universe that is so different from what we have held as true thus far, that it has left many
gasping for air. According to the new theory, it may be that our Universe is not expanding
at all. Rather, galaxies appear to be pushing away from each other on account of a Big
Bang-triggered phenomenon aptly named the Big Wave, which is essentially an expanding
wave flowing through space-time. The team believes that these waves could help explain
why some of the most distant galaxies out there appear to be more distant than they
should be, according to the Standard Model of Cosmology (SM).
"We're saying that maybe these expanding waves are actually causing the anomalous
acceleration. We're saying dark energy is not really the correct explanation," University of
California in Davis (UCD) expert Blake Temple explains. The new set of equations revolves
around Einstein's general theory of relativity, but also seems to offer a decent explanation
for the observed cosmic expansion. Temple worked on the new calculations with University
of Michigan colleague Joel Smoller, and the team published its results in the August 17th
issue of the journal Proceedings of the National Academy of Sciences (PNAS).
"The research could change the way astronomers view the composition of our universe,"
the authors write in the summary of their journal entry, admitting, however, that more
verifications are in order before a final conclusion is drawn. They also say that the new
equations may prove to be a very potent alternative to dark energy theories simply
because the latter were developed hastily, when astronomers discovered that the Universe
was expanding at an ever-increasing speed, and had no explanation for this.
Dark energy "just seems like an unnatural correction to the equations - it's like a fudge
factor. The equations don't make quite as much physical sense when you put it in. You just
put it in to fit the data," Temple says, quoted by Space. "At this stage we think [the new
equations are] a very plausible theory. We're saying there isn't any acceleration. The
qalaxies are displaced from where they're supposed to be because we're in the aftermath


ABSTRACT: In 1927, the American astronomer Edwin Hubble showed the Universe is expanding: distant galaxies are receding from each other. This confirmed the so-called Standard Model of Cosmology, that the universe, on the largest scale, is evolving according to a Friedman-Robertson-Walker spacetime. The starting assumption in this model is the Cosmological Principlethat on the largest scale, we are not in a special place in the universe - that the universe is homogeneous and isotropic about every point like the FRW spacetime. In 1998, more accurate measurements of the recessional velocity of distant galaxies based on new Type 1a supernova data, made the astounding discovery that the Universe was actually accelerating relative to the standard model. So the Standard Model is incorrect. The explanation for the Anomalous Acceleration of the Galaxies is one of the great open problems of physics. 
The only way to account for the Anomalous Acceleration and preserve the FRW framework and the Cosmological Principle is to modify the Einstein equations by adding a Fudge Factor called the Cosmological Constant. Dark Energy, the physical interpretation of the Cosmological Constant, is then an unknown source of anti-gravitation that, for the model to be correct, must account for some 70 percent of the energy density of the universe. This is stated as a fact on the NASA webpage. In this talk I introduce a new family of expanding wave perturbations of the Standard Model, and explore the possibility that these might account for the Anomalous Acceleration of the galaxies without the Cosmological Constant or Dark Energy. [Joint work with Joel Smoller] 


\begin{tabular}{|c|}
\hline $\begin{array}{c}\text { We prove that all of the } \\
\text { self-similar spacetimes } \\
\text { in the family }\end{array}$ \\
$\begin{array}{c}\text { are distinct from the non-critical } \\
k \neq 0 \text { Friedmann spacetimes }\end{array}$ \\
thereby characterizing the \\
critical $k=0$ Friedmann universe as \\
the unique spacetime \\
lying at the intersection \\
of these two one-parameter families.
\end{tabular}


START 
- In the standard model of cosmology, the expanding universe of galaxies evolves from a critically expanding Friedmann Universe $\left(k=0, p=\frac{c^{2}}{3} \rho\right)$

- This is the special case of a

\author{
Non-interacting \\ General Relativistic
}

“Expansion Wave” 
- We show that the standard

Friedmann Universe $\left(k=0, p=\frac{c^{2}}{3} \rho\right)$ can be extended to a 3-parameter family of exact non-interacting expansion waves in GR

- Removing a scaling law and imposing regularity at the center this reduces to a I-parameter family of distict spacetimes that include the standard model, and introduce a correction to the Hubble constant 
- Since non-interacting self-similar expansion waves represent possible timeasymptotic solutions in the theory of conservation laws:

- Q: Could corrections account for the anomalous acceleration of the galaxies w/o cosmological constant/dark energy?

- Q: A new set of solutions to test against the observations? 
INTRODUCTION

TO

COSMOLOGY 


\section{Edwin Hubble (I889-1953)}

- Hubble’s Law (1929):

"The galaxies are receding from us at a velocity proportional to distance"

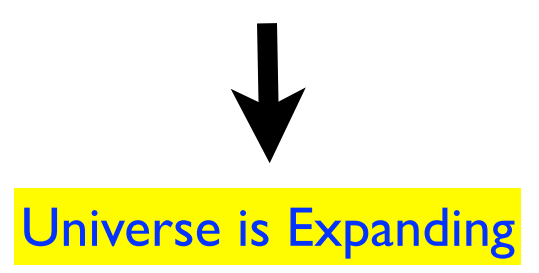

- Based on Redshift vs Luminosity 


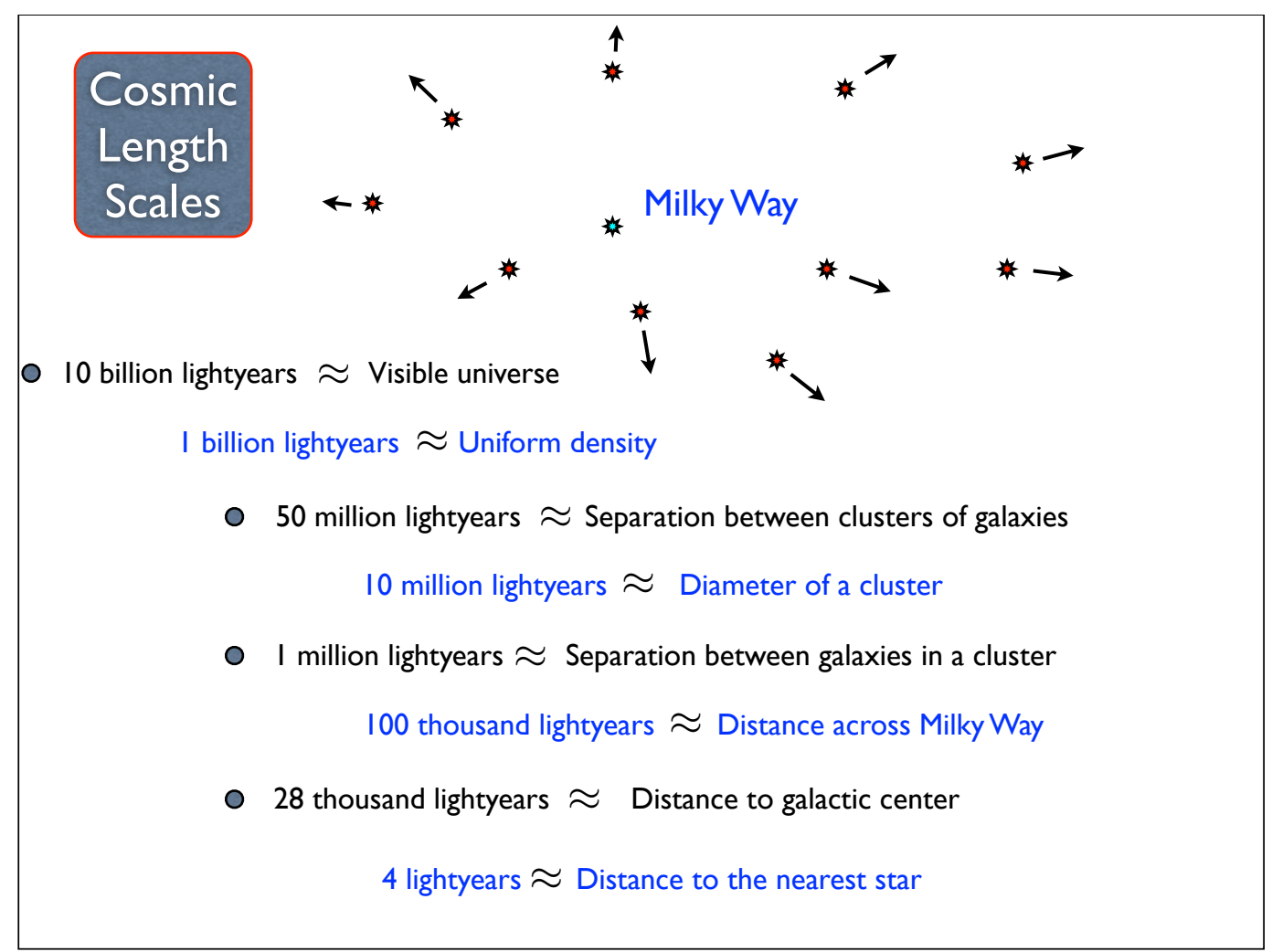




\section{Standard Model of Cosmology}

-1922 Alexander Friedmann:

Derived FRW solutions of the Einstein equations:

3-space of constant curvature expanding in time:

$$
d s^{2}=-d t^{2}+R(t)^{2}\left\{\frac{d r^{2}}{1-k r^{2}}+r^{2} d \Omega^{2}\right\}
$$

- The Big Bang theory based on the FRW metric was worked out by George Lemaitre in the late 1920's leading to Hubble's comfirmation of redshift vs luminoscity consistent with an FRW spacetime

$$
\text { Hubble's Constant } \equiv H \equiv \frac{\dot{R}}{R}
$$


- In 1935: Howard Robertson and Arthur Walker derived FRW from the

\section{Copernican Principle:}

"Earth is not in a special place in the Universe"

- R-W proved: FRW uniquely determined by condition Homogeneous and Isotropic about every point

Any point can be taken as $r=0$

Each $\mathrm{t}=$ const surface is a 3 -space of constant scalar curvature 
Standard Model of Cosmology

Observations of the micro-wave background IMPLY

$$
k=0
$$

"Critical expansion to within about 2-percent" 
The FRW metric when $\mathrm{k}=0$ :

- $\quad d s^{2}=-d t^{2}+R(t)^{2}\left\{d r^{2}+r^{2} d \Omega^{2}\right\}$

The universe is infinite flat space $\mathbb{R}^{3}$ at each fixed time:

- "Galaxies move along $r=$ const., and $\bar{r}=R(t) r$ measures distance at each fixed time" 
The FRW metric when $\mathrm{k}=0$ :

- $\quad d s^{2}=-d t^{2}+R(t)^{2}\left\{d r^{2}+r^{2} d \Omega^{2}\right\}$

The universe is infinite flat space $\mathbb{R}^{3}$ at each fixed time:

"E.g., in Standard Model, during radiation phase, after inflation..."

$$
R(t)=\sqrt{t}
$$




\section{Standard Model of Cosmology}

- FRW metric, $k=0$ :

$$
d s^{2}=-d t^{2}+R(t)^{2}\left\{d r^{2}+r^{2} d \Omega^{2}\right\}
$$

- $D=R r$ Measures distance between galaxies at each fixed $t$

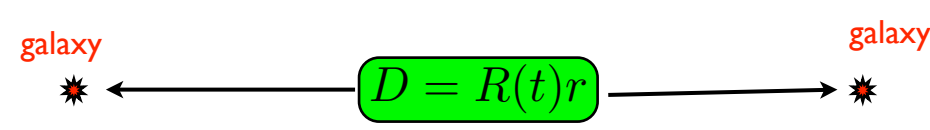

- Conclude: $\quad \dot{D}=\dot{R} r=\frac{\dot{R}}{R} R r=H D$

$$
\dot{D}=H D \backsim \begin{gathered}
\text { Hubble's } \\
\text { Law }
\end{gathered}
$$

$$
\text { Hubble's Constant } \equiv H \equiv \frac{\dot{R}}{R}
$$


- Standard Model of Cosmology

$$
d s^{2}=-d t^{2}+R(t)^{2}\left\{d r^{2}+r^{2} d \Omega^{2}\right\}
$$

- Hubble's Law:

$$
\dot{D}=H D
$$

- Conclude--

"The universe is expanding like a balloon"

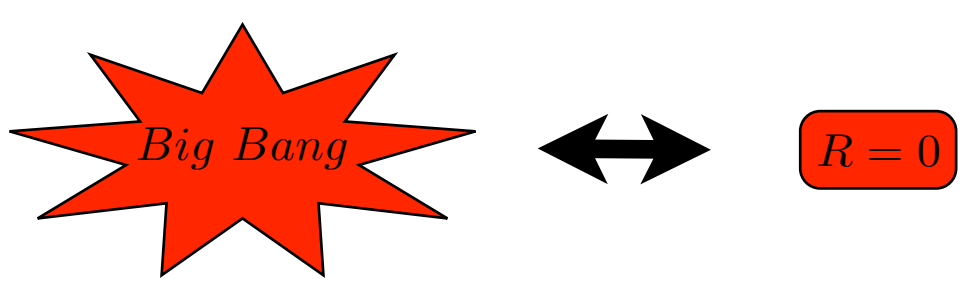


The Hubble "Constant" at present time

$$
H=\frac{\dot{R}}{R} \approx h_{0} \frac{100 \mathrm{~km}}{s \mathrm{mpc}}
$$

- A galaxy at $1 \mathrm{mpc} \approx 3.26$ million lightyears recedes at $h_{0} \frac{100 \mathrm{~km}}{\mathrm{sec}} \quad .5 \leq h_{0} \leq .8$

$$
\frac{1}{H_{0}} \approx 10^{10} \text { years } \approx \text { age of universe }
$$

$\circ \frac{c}{H_{0}} \approx$ Hubble Length $\approx 10^{10}$ lightyears

$\approx$ farthest we can see across the universe 
Recent supernova data have tested the dependence of the Hubble constant on time, and the results don't fit standard model...

$\sqrt{ }$

"Anomalous Acceleration of Galaxies"

Introduction of

"Cosmological Const" and "Dark Energy"

Dark energy is non-classical

Negative pressure $\Rightarrow$ Anti-gravity effect 


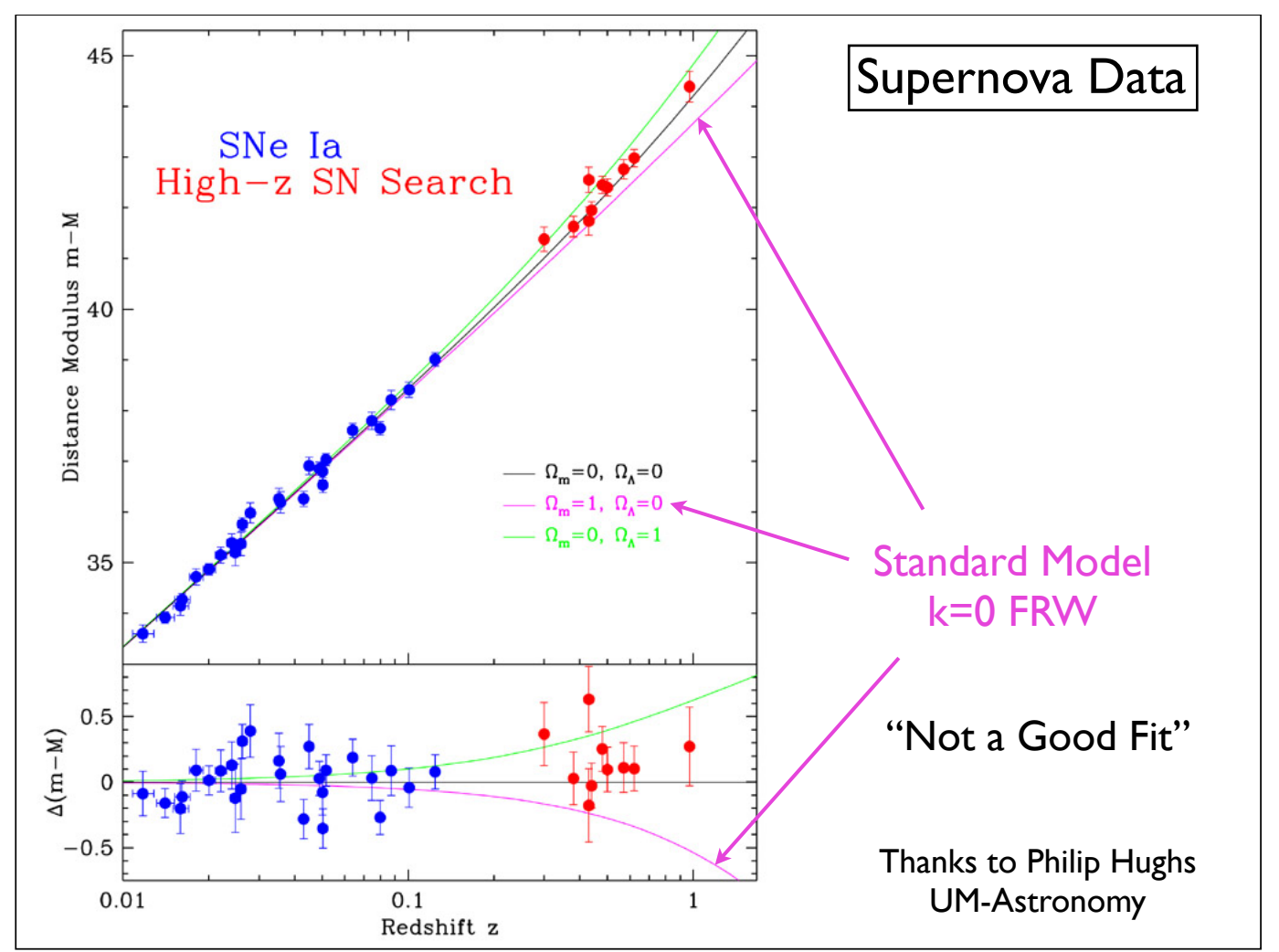




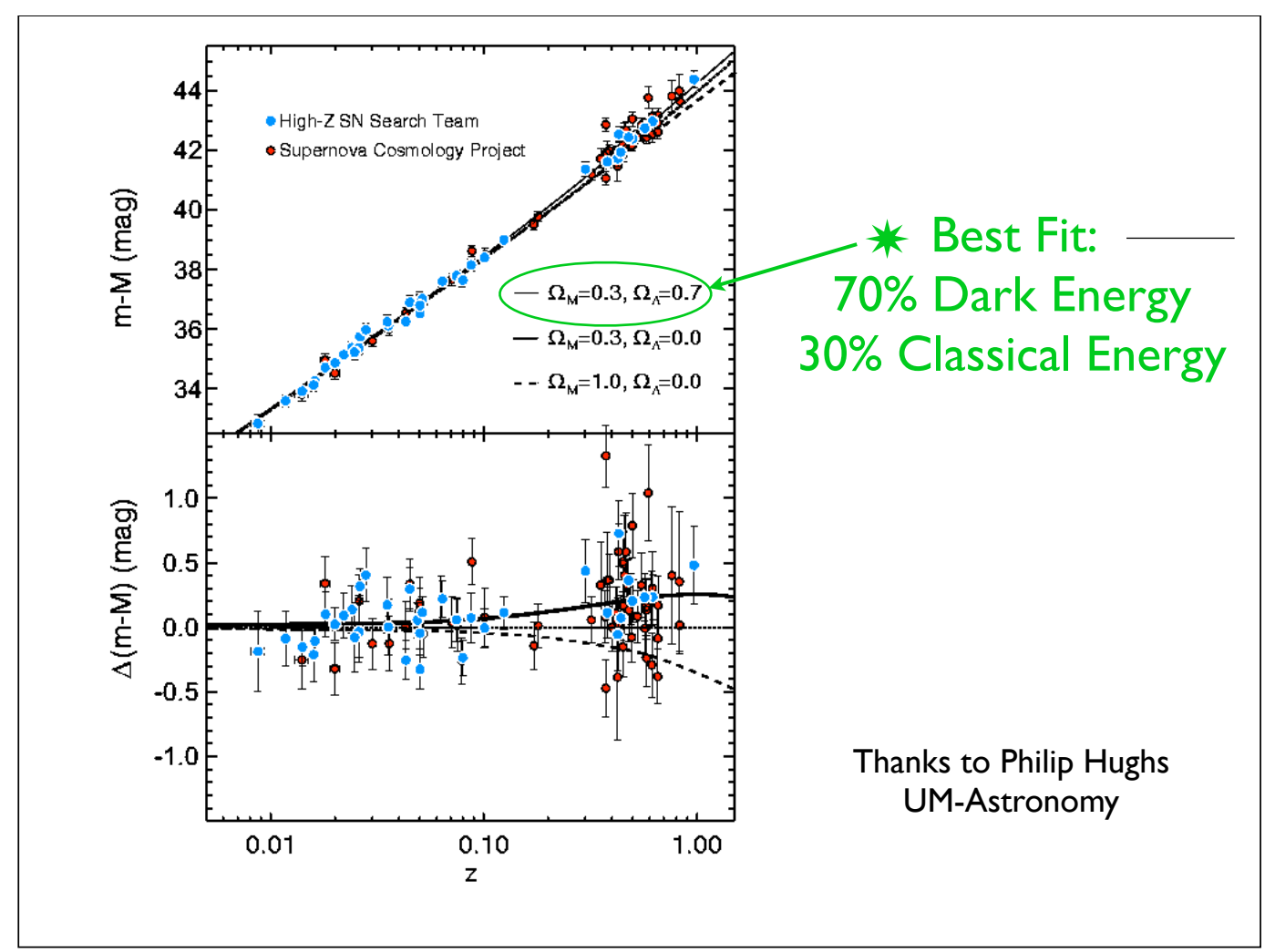




\section{The FRW Mathematical Model:}

- Einstein Equations (19/5): $G_{i j}=\kappa T_{i j}$

$G_{i j}=$ Einstein Curvature Tensor

$T_{i j}=(\rho+p) u_{i} u_{j}+p g_{i j}=$ Stress Energy Tensor (perfect fluid)

- Einstein Equations for $k=0$ Friedmann metric:

$$
\begin{aligned}
& H^{2}=\frac{\kappa}{3} \rho \\
& \dot{\rho}=-3(\rho+p) H
\end{aligned}
$$

Solutions determined by equation of state: $p=p(\rho)$ 


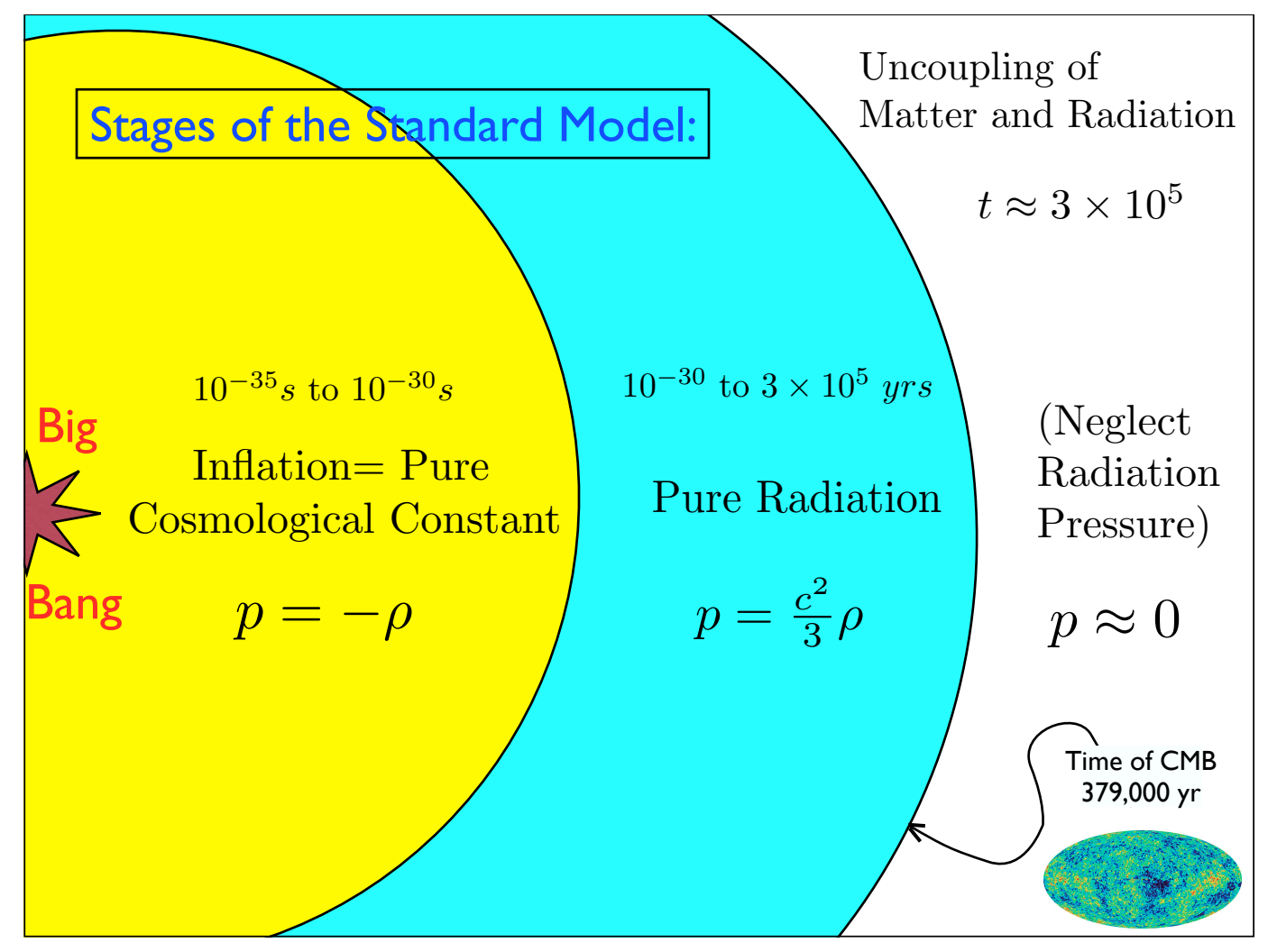




\section{Standard Model for Dark Energy}

- Assume Einstein equations with a cosmological constant:

$$
G_{i j}=8 \pi T_{i j}+\Lambda g_{i j}
$$

- Assume $k=0$ FRW: $d s^{2}=-d t^{2}+R(t)^{2}\left\{d r^{2}+r^{2} d \Omega^{2}\right\}$

- Leads to:

$$
H^{2}=\frac{\kappa}{3} \rho+\frac{\kappa}{3} \Lambda
$$

○ Divide by $H^{2}=\frac{\kappa}{3} \rho_{\text {crit }}$

$$
1=\Omega_{M}+\Omega_{\Lambda}
$$

- Best data fit leads to $\Omega_{\Lambda} \approx .73$ and $\Omega_{M} \approx .27$

- Implies: The universe is $\mathbf{7 3}$ percent dark energy 


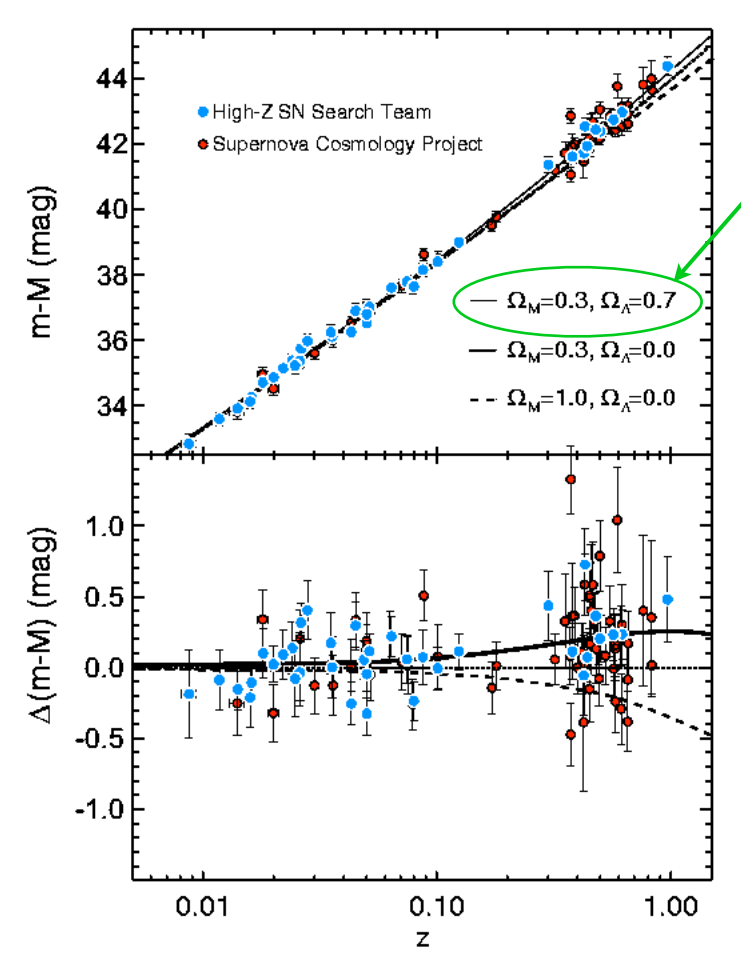

\section{0\% Dark Energy} 30\% Classical Energy

- $\mathrm{m}-\mathrm{M}=$ "Distance Modulus" $\mathrm{M}=$ absolute Magnitude m=apparent magnitude

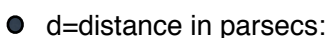
$m-M=5 \log (d)-5$

$z=$ redshift factor $1+z=\frac{\lambda_{\text {emit }}}{\lambda_{o b s}}$

- $\Omega_{m}+\Omega_{\Lambda}=1$ for a flat $(k=0)$ universe. 


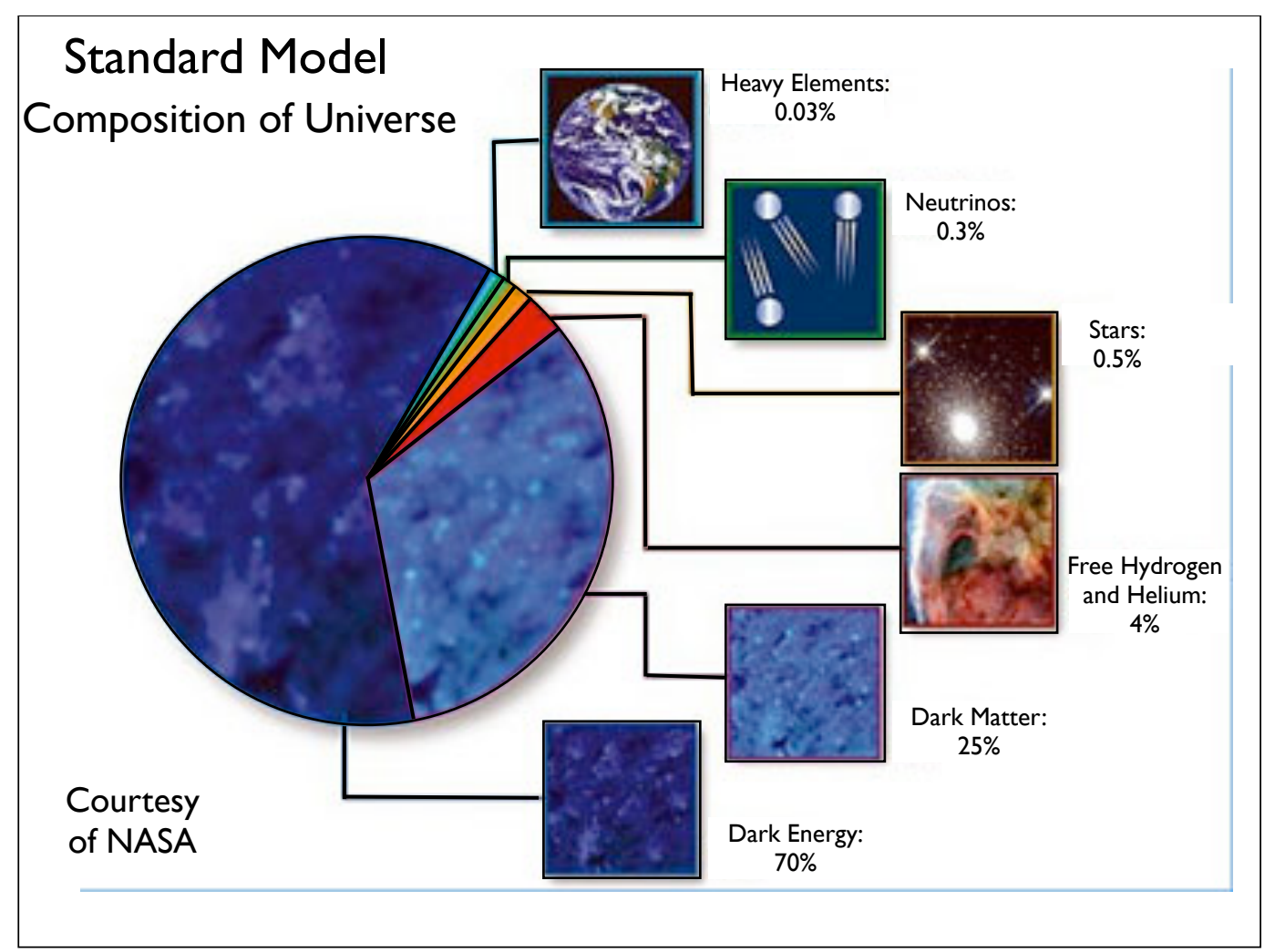




\section{The Question we Explore:}

"Could the anomalous acceleration of the galaxies be due to the fact that we are looking outward into an expansion wave different from the $k=0 \mathrm{FRW}$ spacetime, and NOT due to a cosmological constant?" 
The Question we Explore:

"Could the anomalous acceleration of the galaxies be due to the fact that we are looking out into an expansion wave different from the $k=0 \mathrm{FRW}$ spacetime, and NOT due to a cosmological constant?"

The Einstein equations have been confirmed without the cosmological constant in every setting except cosmology... 
The Question we Explore:

"Could the anomalous acceleration of the galaxies be due to the fact that we are looking out into an expansion wave different from the $k=0 \mathrm{FRW}$ spacetime, and NOT due to a cosmological constant?"

The Einstein equations have been confirmed without the cosmological constant in every setting except cosmology...

Note: A general expansion wave has a center of expansion... 
The Einstein equations that describe the expansion of the Universe during the radiation phase of the expansion form a highly nonlinear system of coupled wave equations in the form of conservation laws. 


\begin{tabular}{|c|}
\hline Such wave equations support the \\
propagation of waves, \\
and self-similar expansion waves are \\
important because even when dissipative \\
terms are neglected in conservation laws, \\
the nonlinearities alone provide a \\
mechanism whereby non-interacting self- \\
similar wave patterns can emerge from \\
general interactive solutions, via the \\
process of wave interaction and shock \\
wave dissipation
\end{tabular}




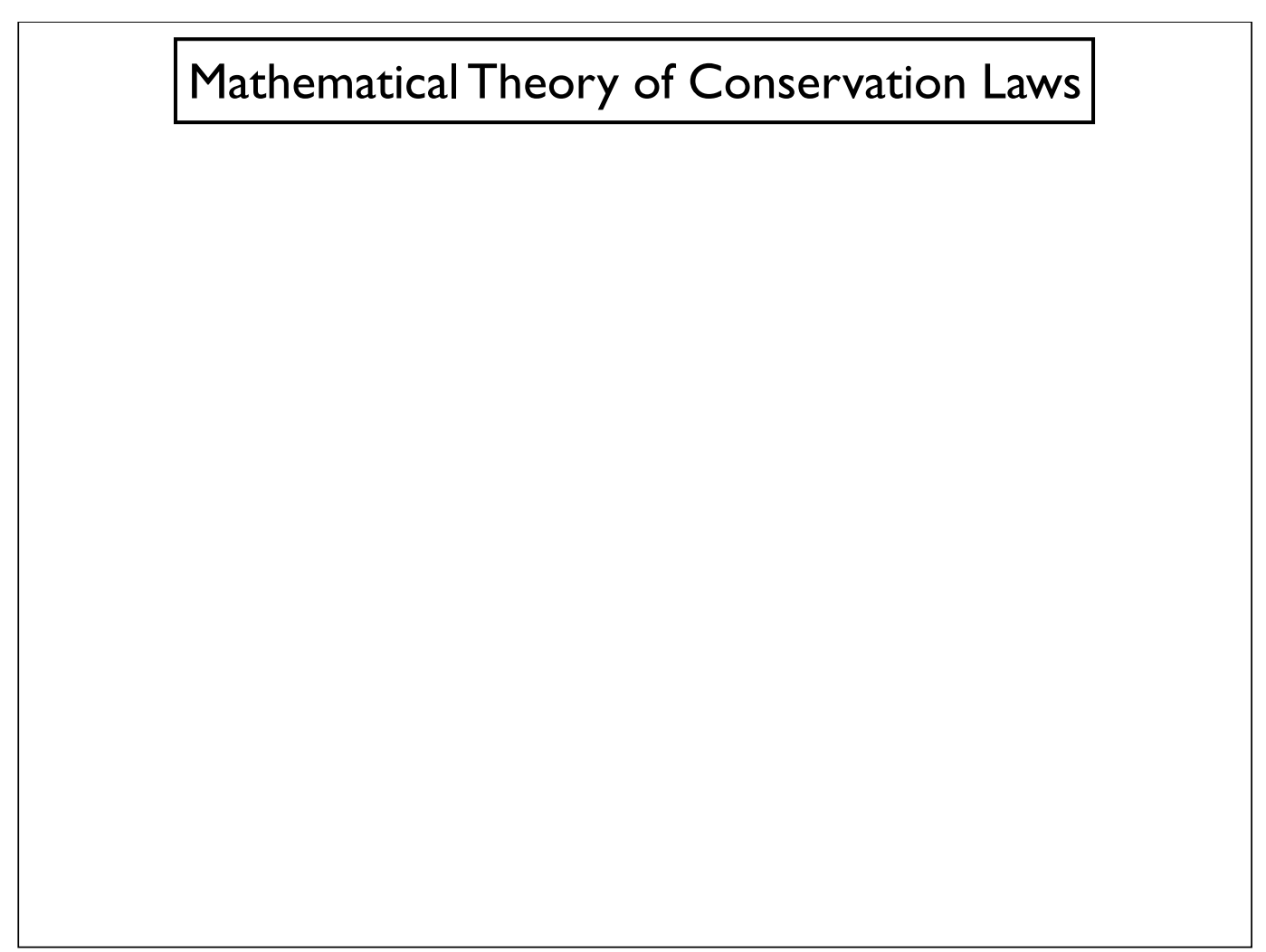




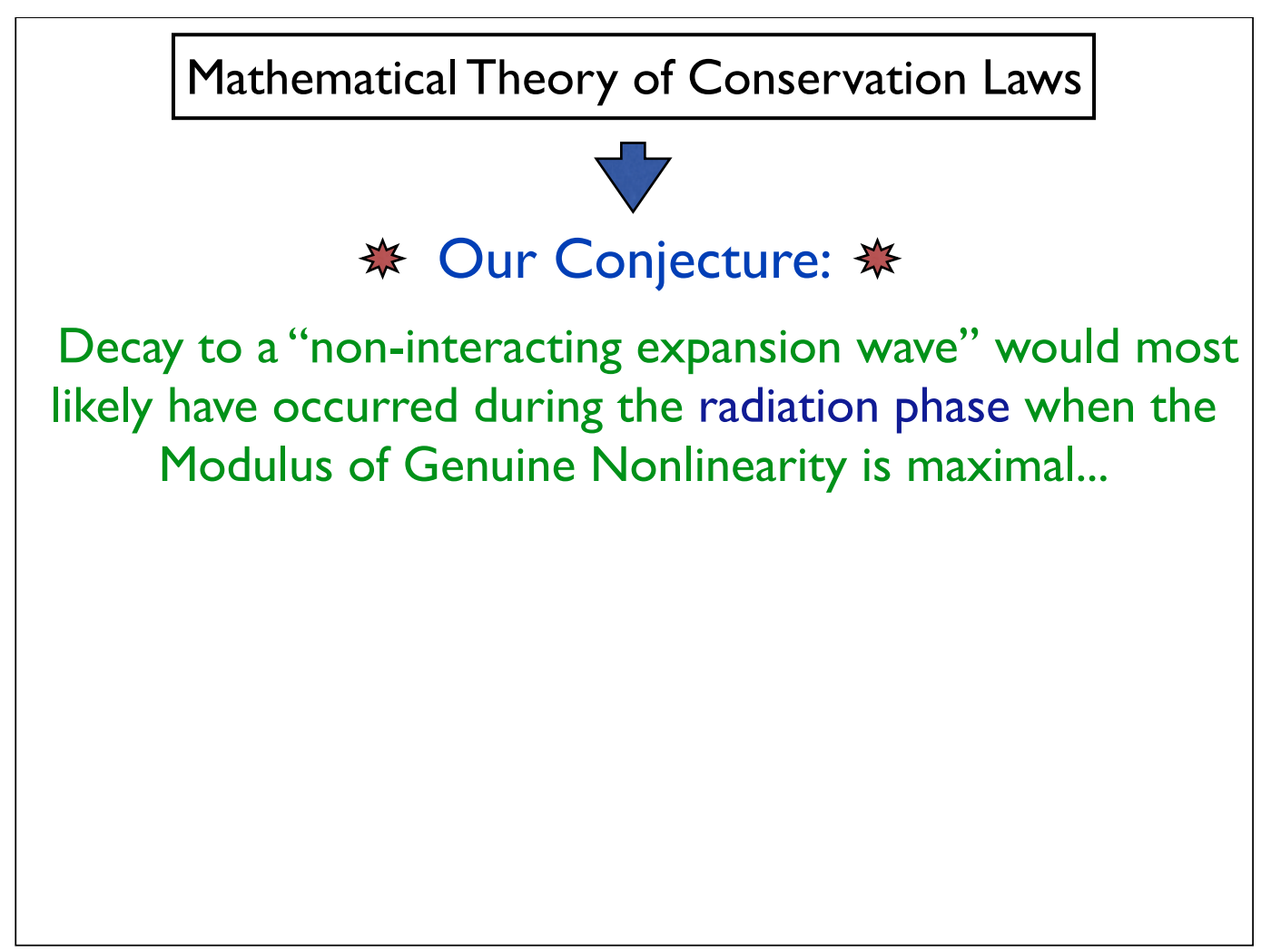




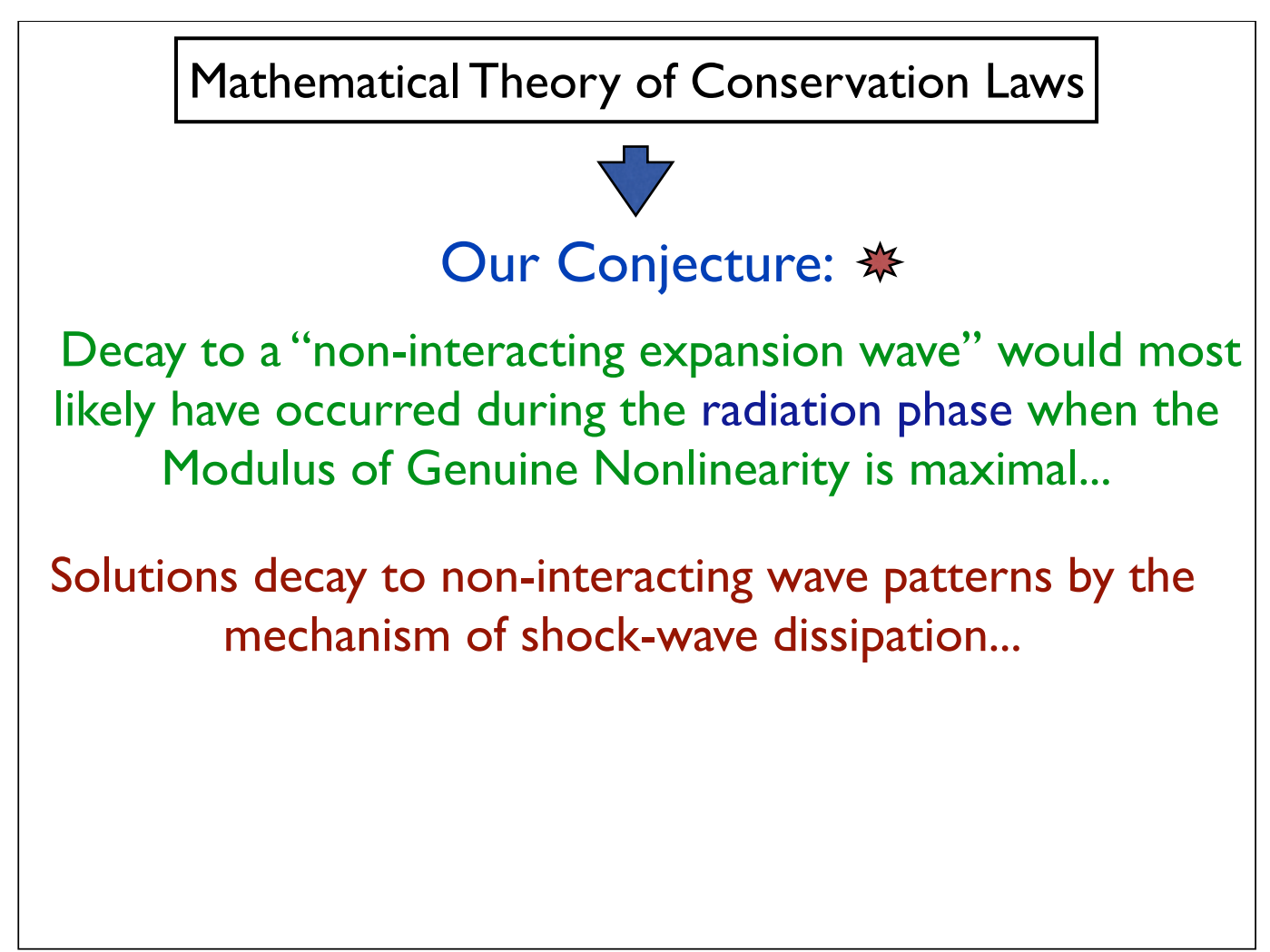




\begin{tabular}{l}
\hline Mathematical Theory of Conservation Laws \\
Decay to a "non-interacting expansion wave" would most \\
likely have occurred during the radiation phase when the \\
Modulus of Genuine Nonlinearity is maximal... \\
Solutions decay to non-interacting wave patterns by the \\
mechanism of shock-wave dissipation... \\
DECAY OCCURS EVEN WHEN \\
DISSIPATIVE TERMS ARE NEGLECTED \\
(A Subtle Point!)
\end{tabular}


- Basic warmup problem: scalar Burgers Equation:

$$
u_{t}+u u_{x}=0
$$

Decay to non-interacting simple waves by "shock wave dissipation"

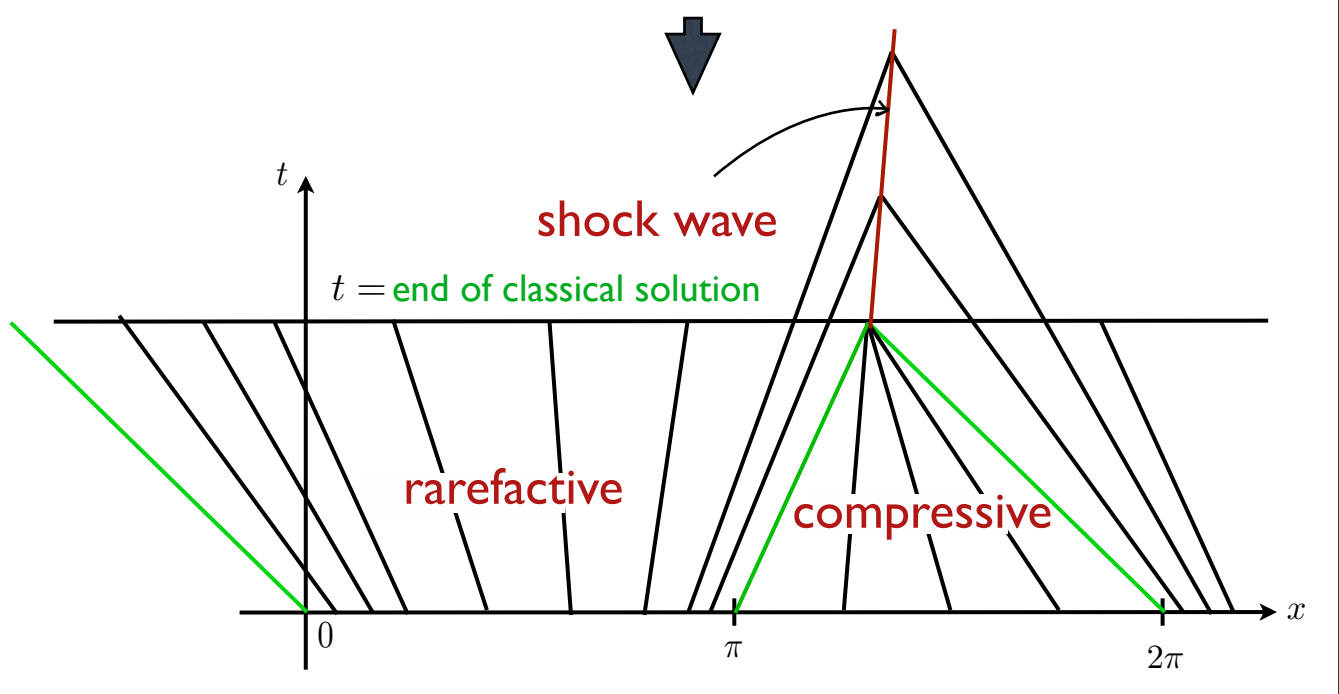




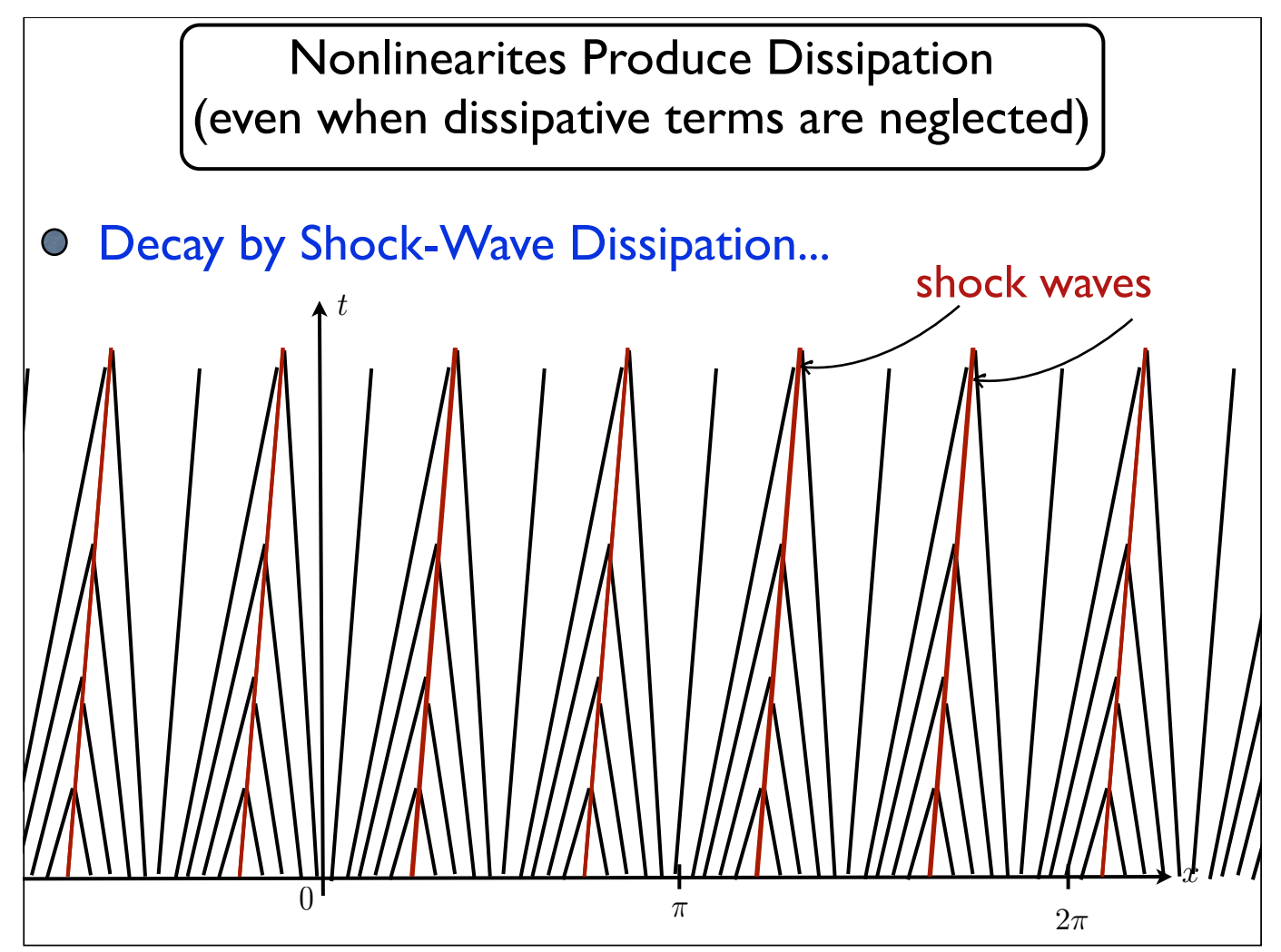




\begin{tabular}{|l|}
\hline Compare: \\
\hline O Pure Radiation: $\quad p=\frac{c^{2}}{3} \rho$ \\
O Matter Dominated: $\quad p=0$ \\
\end{tabular}




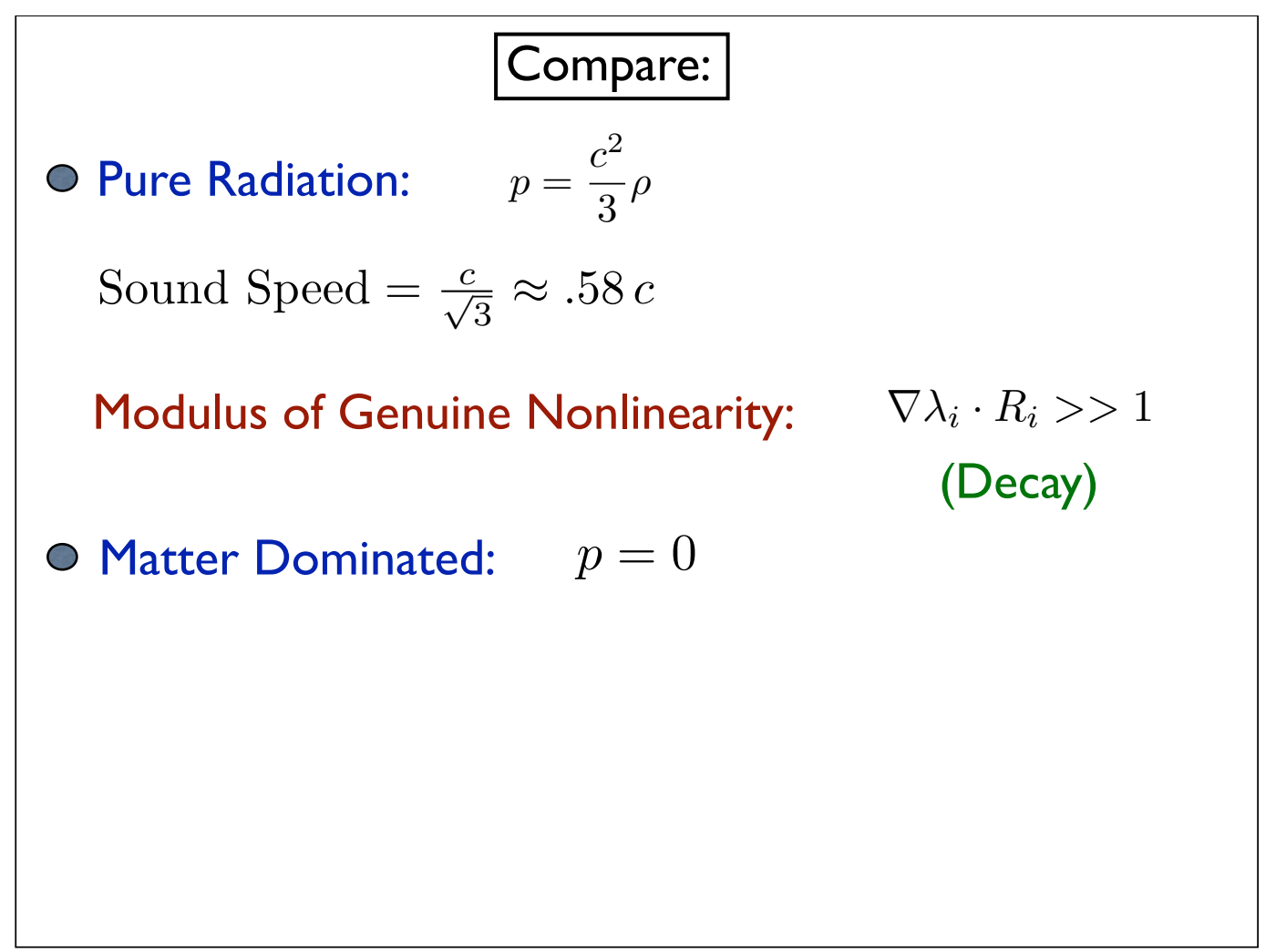




\begin{tabular}{|c|c|}
\hline Compare: & \\
\hline Pure Radiation: $\quad p=\frac{c^{2}}{3} \rho$ & \\
\hline Sound Speed $=\frac{c}{\sqrt{3}} \approx .58 c$ & \\
\hline Modulus of Genuine Nonlinearity: & $\begin{array}{c}\nabla \lambda_{i} \cdot R_{i}>>1 \\
(\text { Decay })\end{array}$ \\
\hline Matter Dominated: $\quad p=0$ & \\
\hline Sound Speed $\equiv 0$ & \\
\hline Modulus of Genuine Nonlinearity: & $\begin{array}{l}\nabla \lambda_{i} \cdot R_{i} \equiv 0 \\
\text { (No Decay) }\end{array}$ \\
\hline
\end{tabular}




\section{To start: we proposed to numerically simulate the secondary reflected wave reflected back in our shock wave cosmology model... \\ References: \\ O Talk: Numerical Cosmology Session, National meeting, New Orleans, January 2007 http://www.math.ucdavis.edu/ temple/ \\ OThesis: numerical simulation by a locally inertial Godunov method, Zeke Volger, UC-Davis, 2009}




\section{Could the Anomalous acceleration be accounted for}

by an expansion behind the Shock Wave?

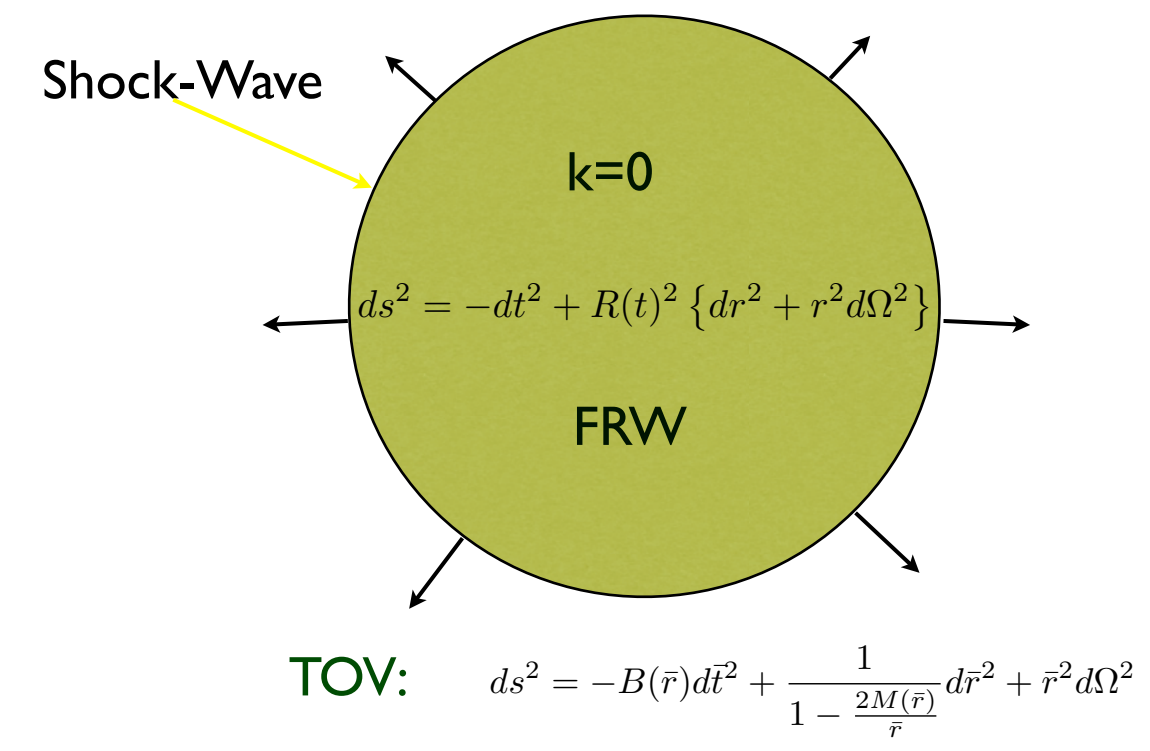




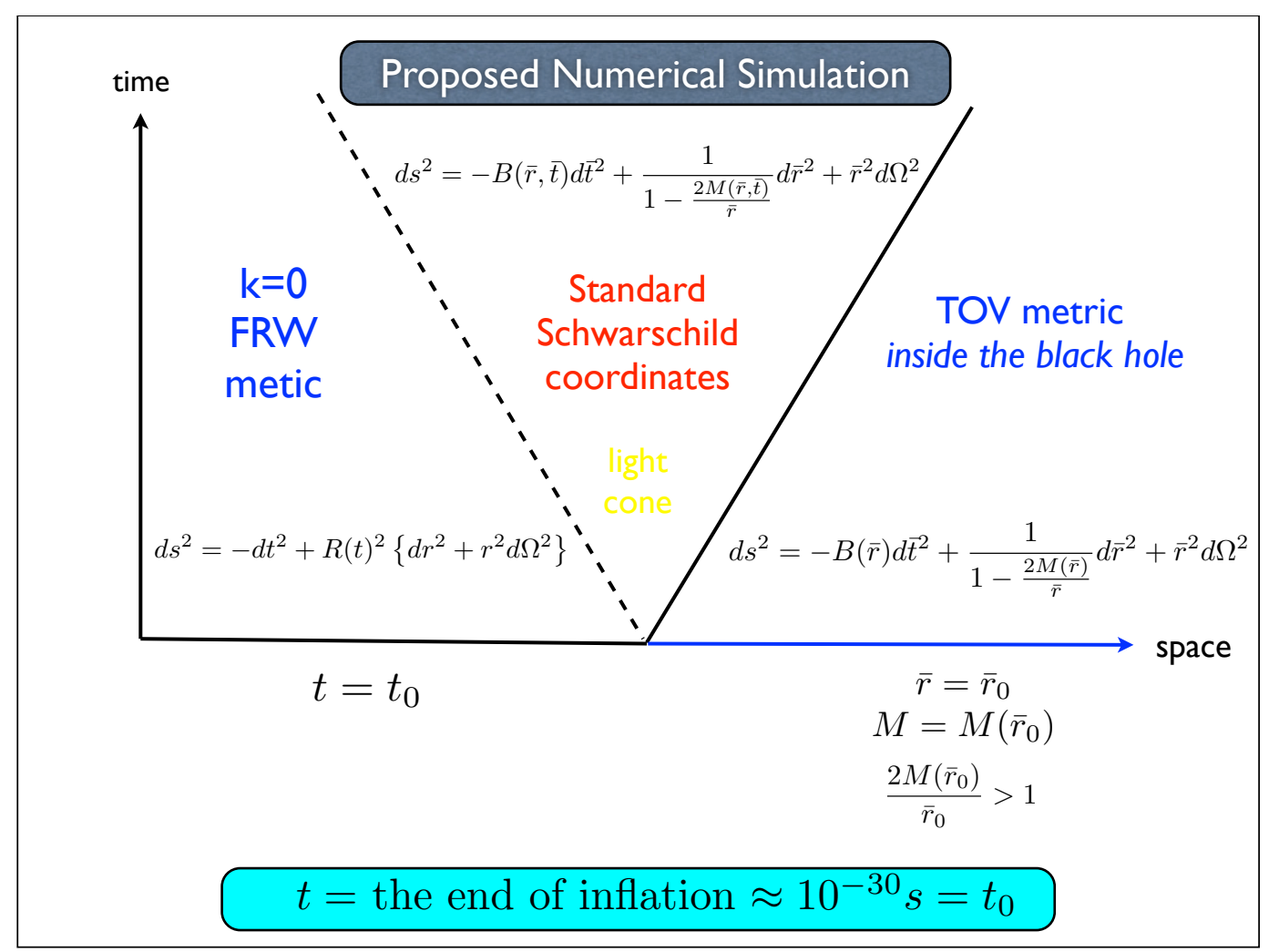


- The numerical method required getting an explicit form for the $(k=0, p=1 / 3 \rho)$-FRW metric

Standard Schwarzschild Coordinates 
- The numerical method required getting an explicit form for the $(k=0, p=1 / 3 \rho)$-FRW metric

Standard Schwarzschild Coordinates

- Upon doing this we found that there exists an integrating factor such that the metric satisfies an ODE in Standard Schwarzschild coordinates...the ODE's then introduce 3 extra free parameters...

...(the 3 -initial conditions)! 
Thus: we look for an expanding wave perturbation of the $k=0$ FRW metric during the period when:

$$
\begin{gathered}
p=\frac{c^{2}}{3} \rho \\
d s^{2}=-d t^{2}+R(t)^{2}\left\{d r^{2}+r^{2} d \Omega^{2}\right\} \\
\boldsymbol{v} \\
R(t)=\sqrt{t} \\
H(t)=\frac{\dot{R}(t)}{R(t)}=\frac{1}{2 t}
\end{gathered}
$$




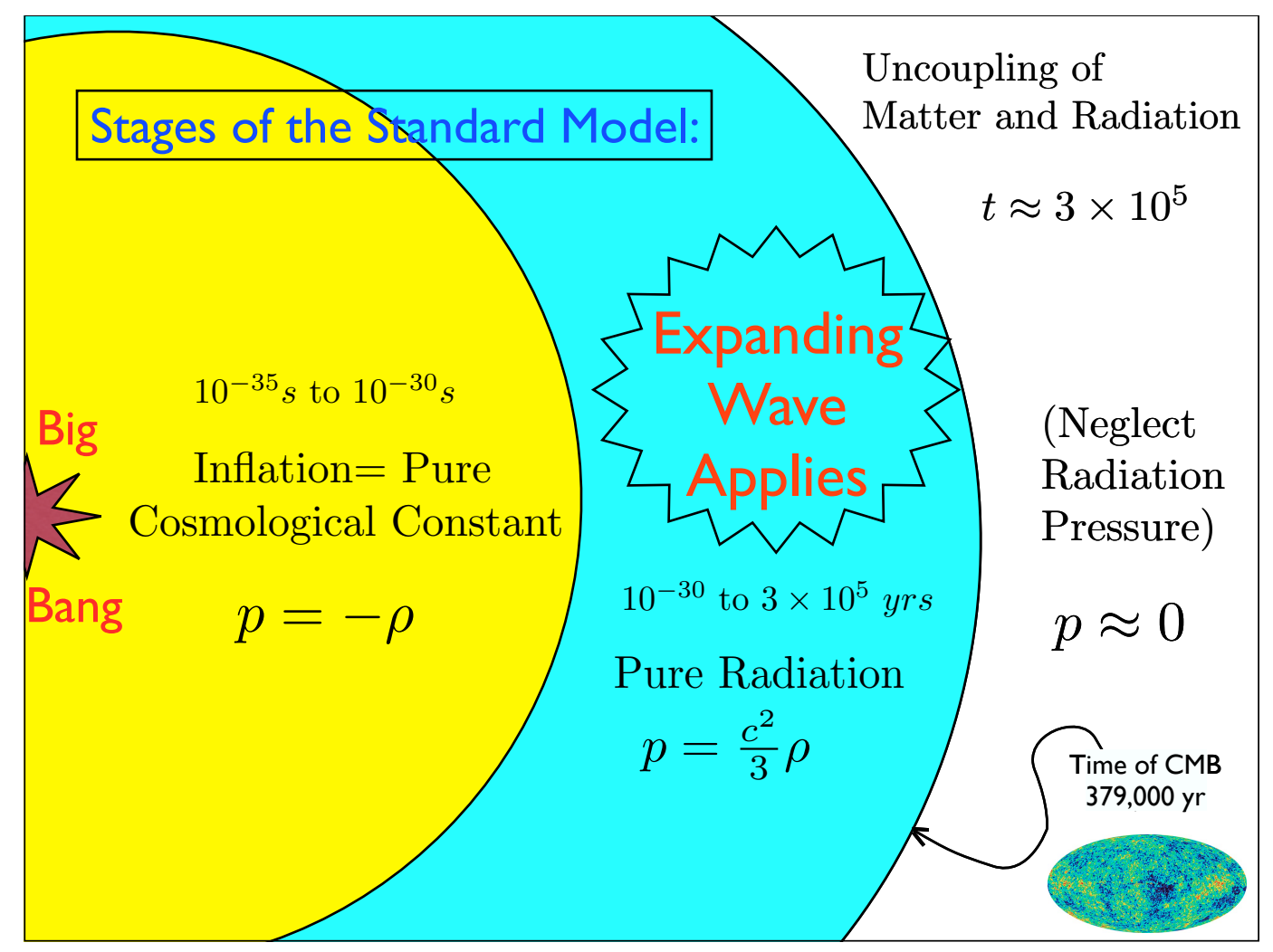


A Three Parameter Family of Expanding Wave Solutions of the

Einstein Equations including

The Standard Model of

Cosmology 


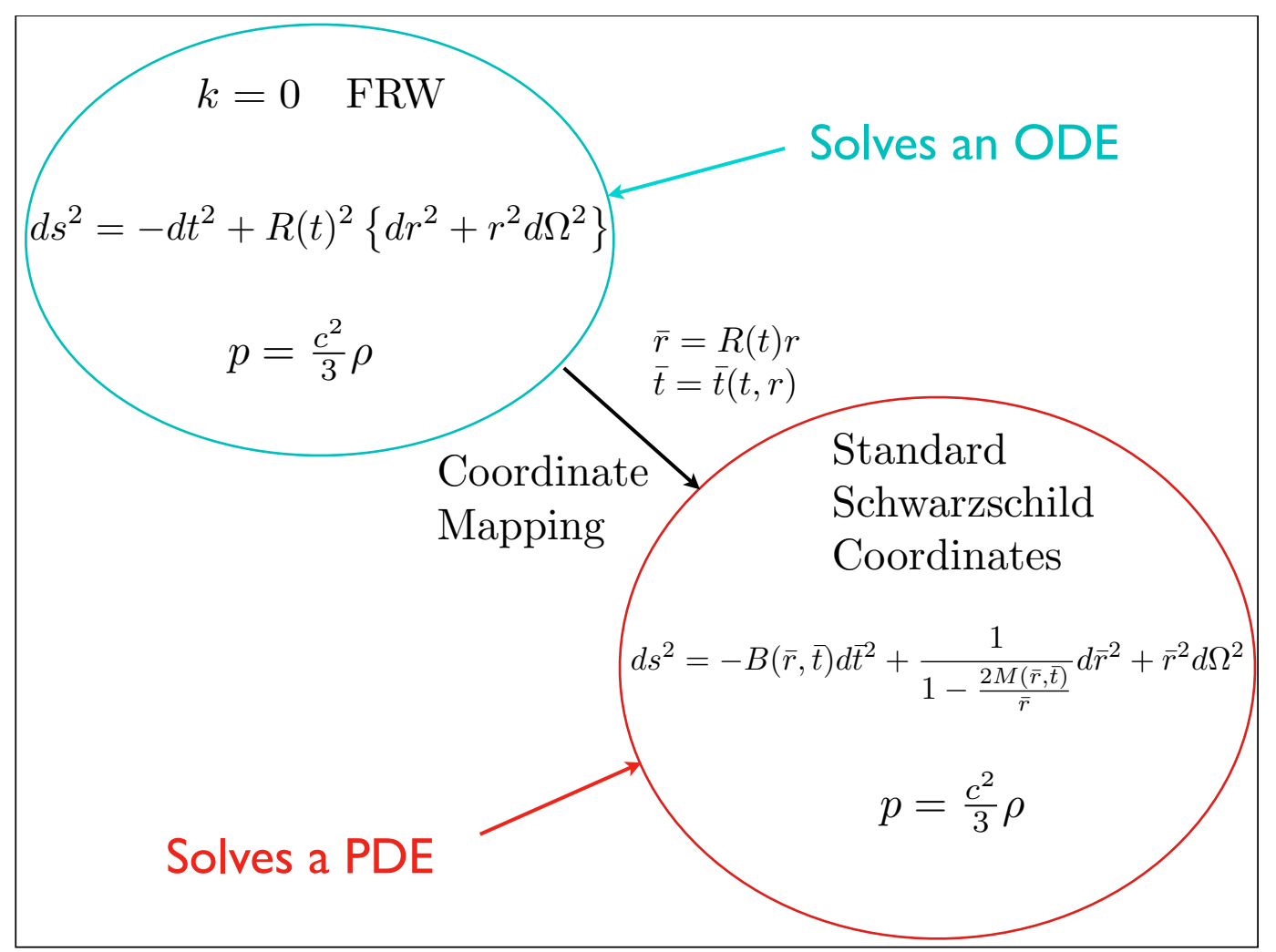


- Spherically symmetric spacetime metrics can "generically" be mapped over to

Standard Schwarzschild Coordinates... [c.f.Wein]

- In general there exist MANY ways

to do this, depending on an INTEGRATING FACTOR that solves a PDE 
Theorem: Assume $p=\frac{c^{2}}{3} \rho, k=0$. Then the FRW metric

$$
d s^{2}=-d t^{2}+R(t)^{2} d r^{2}+\bar{r}^{2} d \Omega^{2},
$$

under the mapping

$$
\begin{aligned}
& \bar{r}=R(t) r, \\
& \bar{t}=\left\{1+\left[\frac{R(t) r}{2 t}\right]^{2}\right\} t,
\end{aligned}
$$

goes over to the SSC-metric

$$
d s^{2}=-\frac{d \bar{t}^{2}}{1-v(\xi)^{2}}+\frac{d \bar{r}^{2}}{1-v(\xi)^{2}}+\bar{r}^{2} d \Omega^{2},
$$

where

$$
\xi \equiv \frac{\bar{r}}{\bar{t}}=\frac{2 v}{1+v^{2}}
$$


Corollary: There exists a coordinate mapping that takes the $p=\frac{1}{3} \rho, k=0$ FRW metric over to SSC-coordinates such that SSC metric components

DEPEND ONLY ON THE SINGLE VARIABLE

$$
\xi=\frac{\bar{r}}{\bar{t}}
$$

(Like an expansion wave!) 
This implies that the standard FRW metric after inflation is equivalent to a metric that satisfies an ODE in SSC-Coordinates! 
We now construct this ODE systematically... 


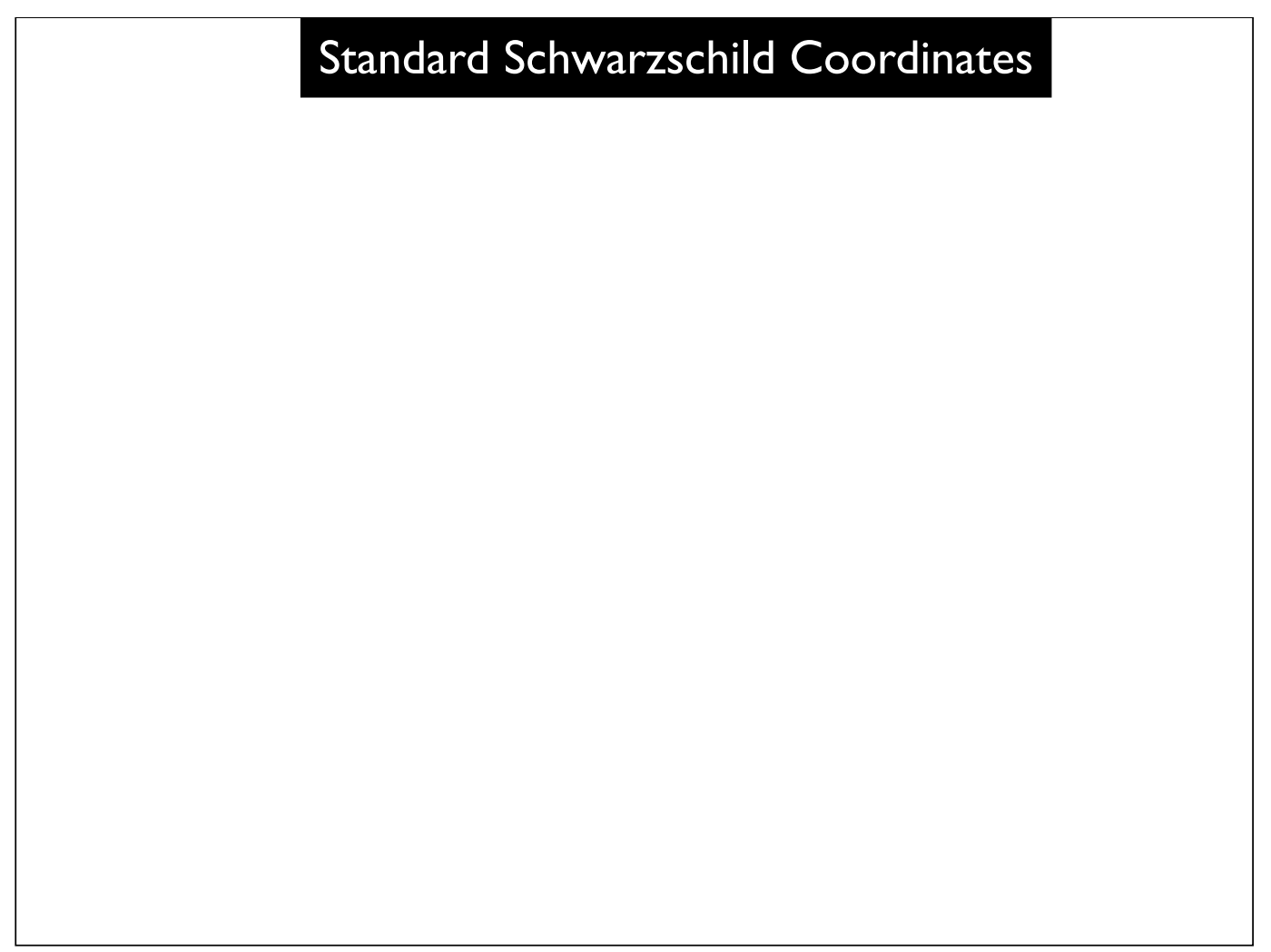




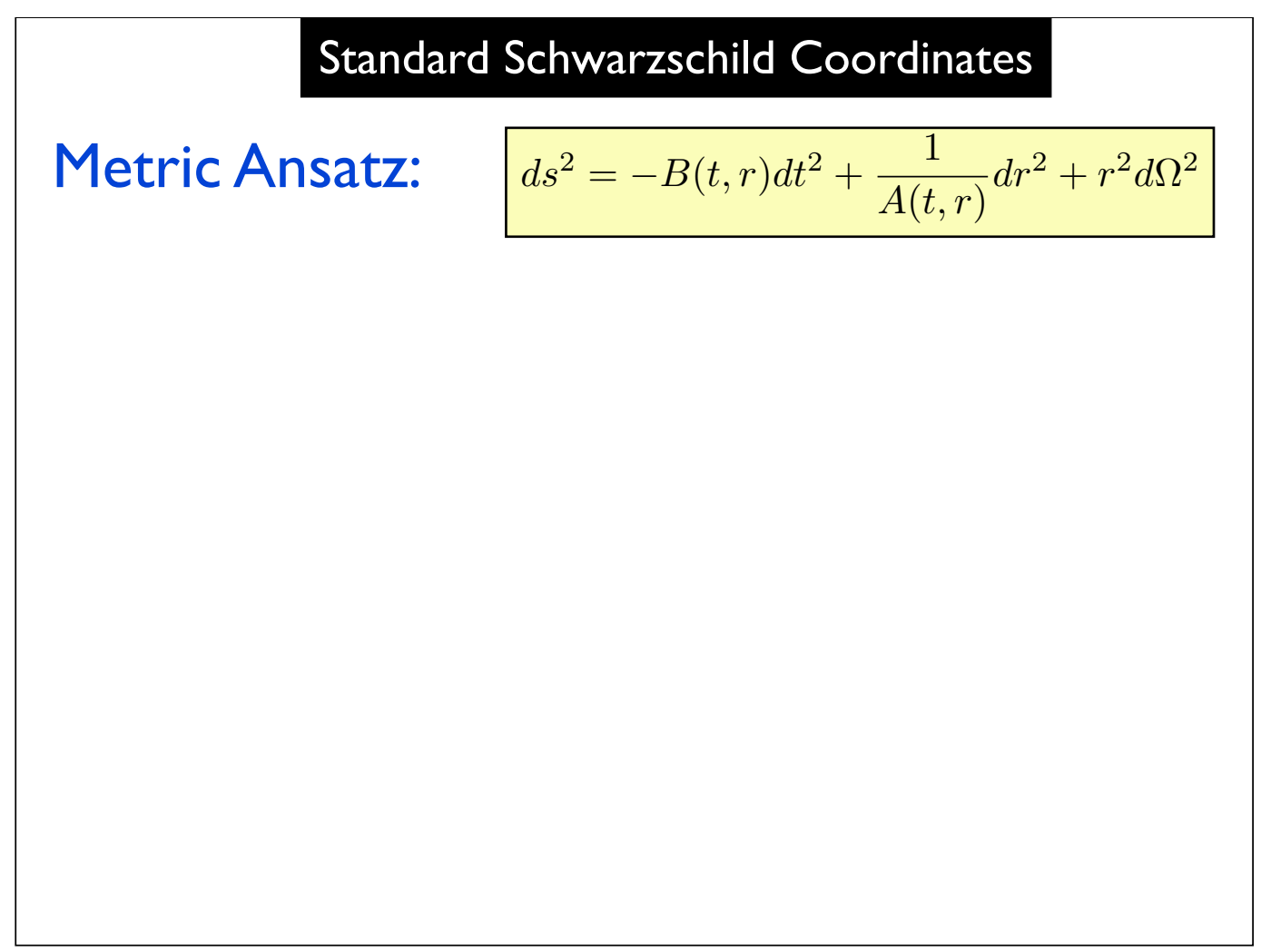




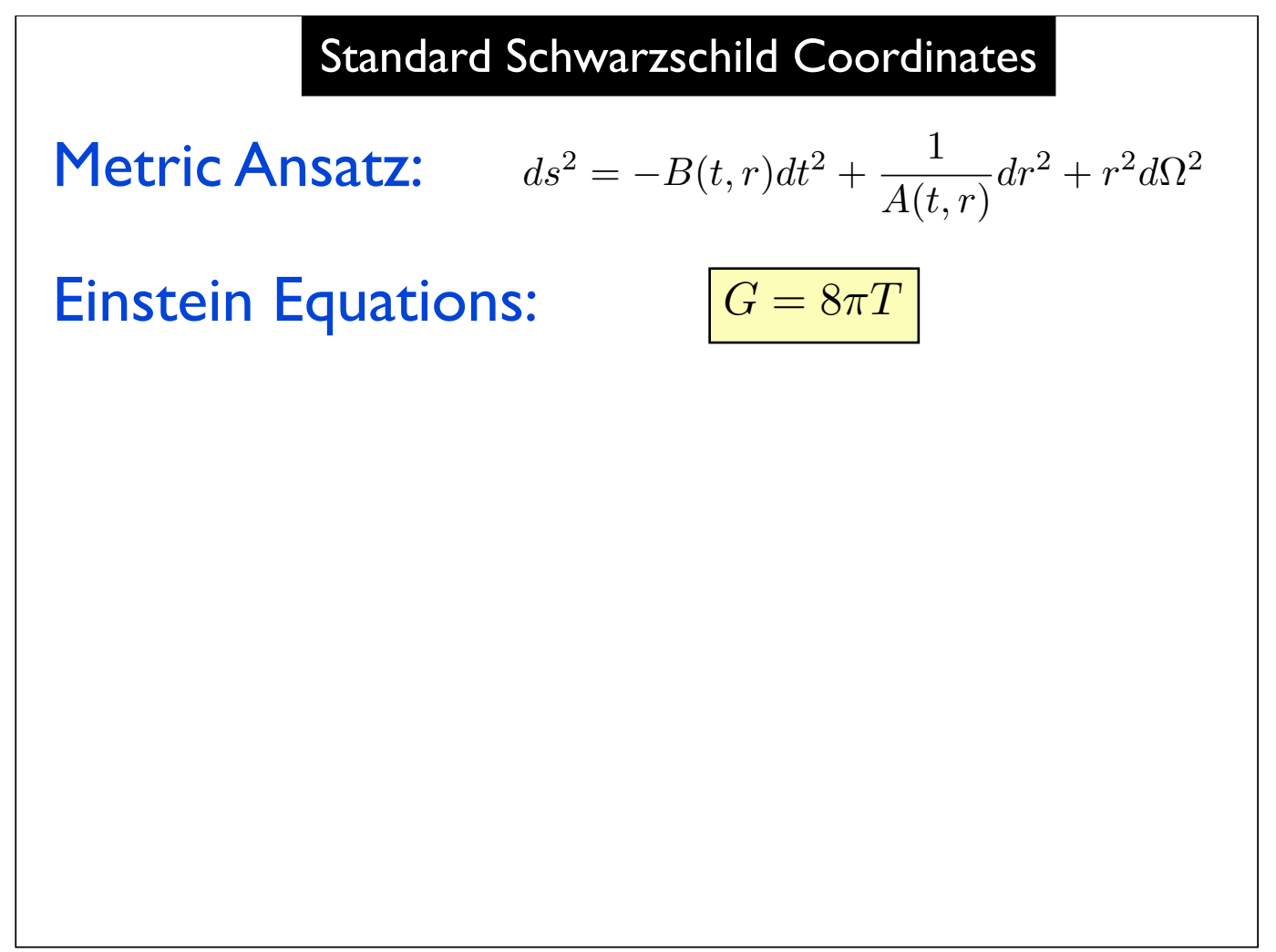




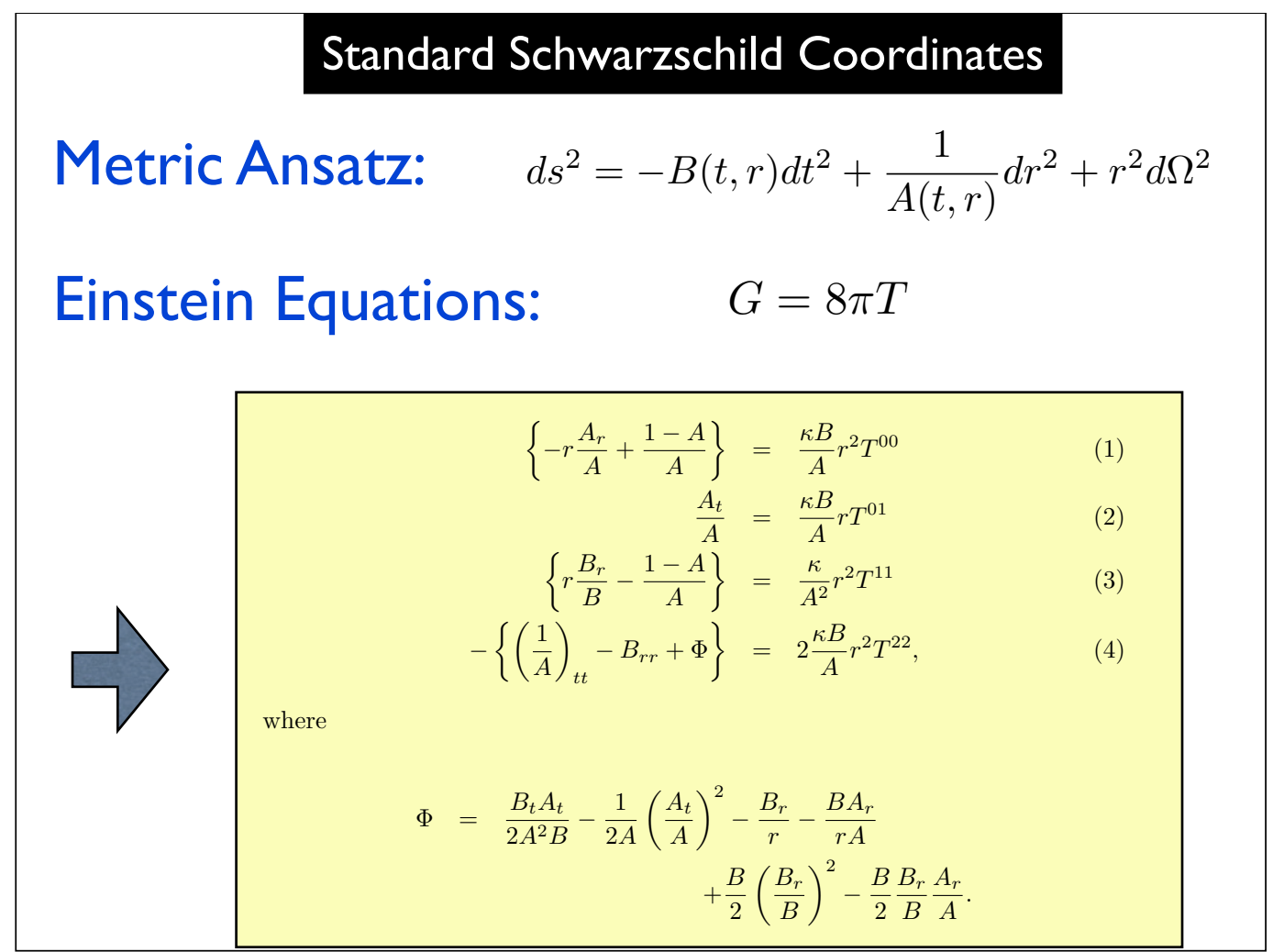


Q: When do the SSC PDE's reduce to ODE's?

$$
\begin{aligned}
& \begin{array}{c}
\text { Four } \\
\text { PDE's }
\end{array} \\
& \left\{-r \frac{A_{r}}{A}+\frac{1-A}{A}\right\}=\frac{\kappa B}{A} r^{2} T^{00} \\
& \frac{A_{t}}{A}=\frac{\kappa B}{A} r T^{01} \\
& \left\{r \frac{B_{r}}{B}-\frac{1-A}{A}\right\}=\frac{\kappa}{A^{2}} r^{2} T^{11} \\
& -\left\{\left(\frac{1}{A}\right)_{t t}-B_{r r}+\Phi\right\}=2 \frac{\kappa B}{A} r^{2} T^{22}, \\
& \Phi=\frac{B_{t} A_{t}}{2 A^{2} B}-\frac{1}{2 A}\left(\frac{A_{t}}{A}\right)^{2}-\frac{B_{r}}{r}-\frac{B A_{r}}{r A} \\
& +\frac{B}{2}\left(\frac{B_{r}}{B}\right)^{2}-\frac{B}{2} \frac{B_{r}}{B} \frac{A_{r}}{A} .
\end{aligned}
$$

Ans\#I: $A=A(r), B=B(r)$ time-independent 
$A=A(r), B=B(r)$ time-independent

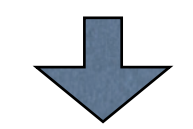

Oppenheimer-Volkoff equations for a

Static Fluid Sphere

(The setting for the stability limits in stars)

---Buchdahl Stability Limit

---Chandresekhar Stability Limit 
The Oppenheimer-Volkoff equations:

$$
\begin{aligned}
A^{\prime}(r) & =\frac{1-A}{r}-\kappa r \\
\frac{B^{\prime}(r)}{B} & =-2 \frac{p^{\prime}(r)}{p+\rho} \\
p^{\prime}(r) & =-\frac{\mathcal{G} M \rho}{r^{2}}\left\{1+\frac{p}{\rho}\right\}\left\{1+\frac{4 \pi r^{3} p}{M}\right\}\left\{1-\frac{2 \mathcal{G} M}{r}\right\}^{-1}
\end{aligned}
$$

...the fundamental equation of Newtonian astrophysics, with general-relativistic corrections supplied by the last three factors, [Weinberg, page 301]. 
We show there is another way the SSC-Equations reduce to ODE's:

I.e., when

(I) $\quad T_{i j}=(\rho+p) u^{i} u^{j}+p g^{i j}$ is linear in $\rho$

(2) $\quad A, B, v$ and $r^{2} \rho$ depend on $\xi=r / t$

This includes the case

$$
p=\frac{c^{2}}{3} \rho
$$


The SSC-equations reduce to ODE's when:

(I) $T_{i j}=(\rho+p) u^{i} u^{j}+p g^{i j}$ is linear in $\rho$

(2) $A, B, v$ and $r^{2} \rho$ depend on $\xi=r / t$

Claim: one choice of initial conditions gives the standard model! 


\section{We now see how this works:}




\section{Standard Schwarzschild Coordinates}

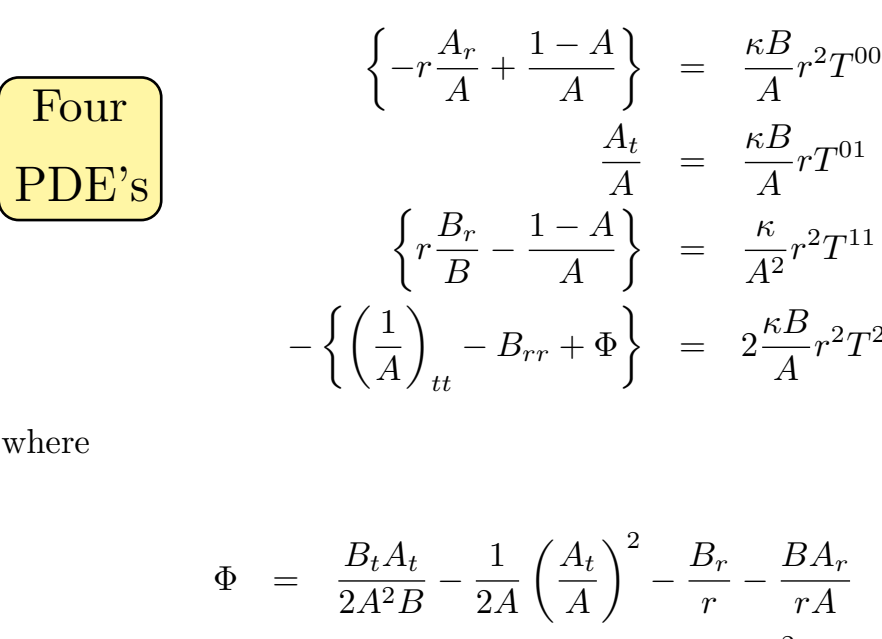

$$
\begin{aligned}
& +\frac{B}{2}\left(\frac{B_{r}}{B}\right)^{2}-\frac{B}{2} \frac{B_{r}}{B} \frac{A_{r}}{A} . \\
& (I)+(2)+(3)+(4) \quad \sum_{\text {(weakly) }}(1)+(3)+\operatorname{div} \mathrm{T}=0
\end{aligned}
$$




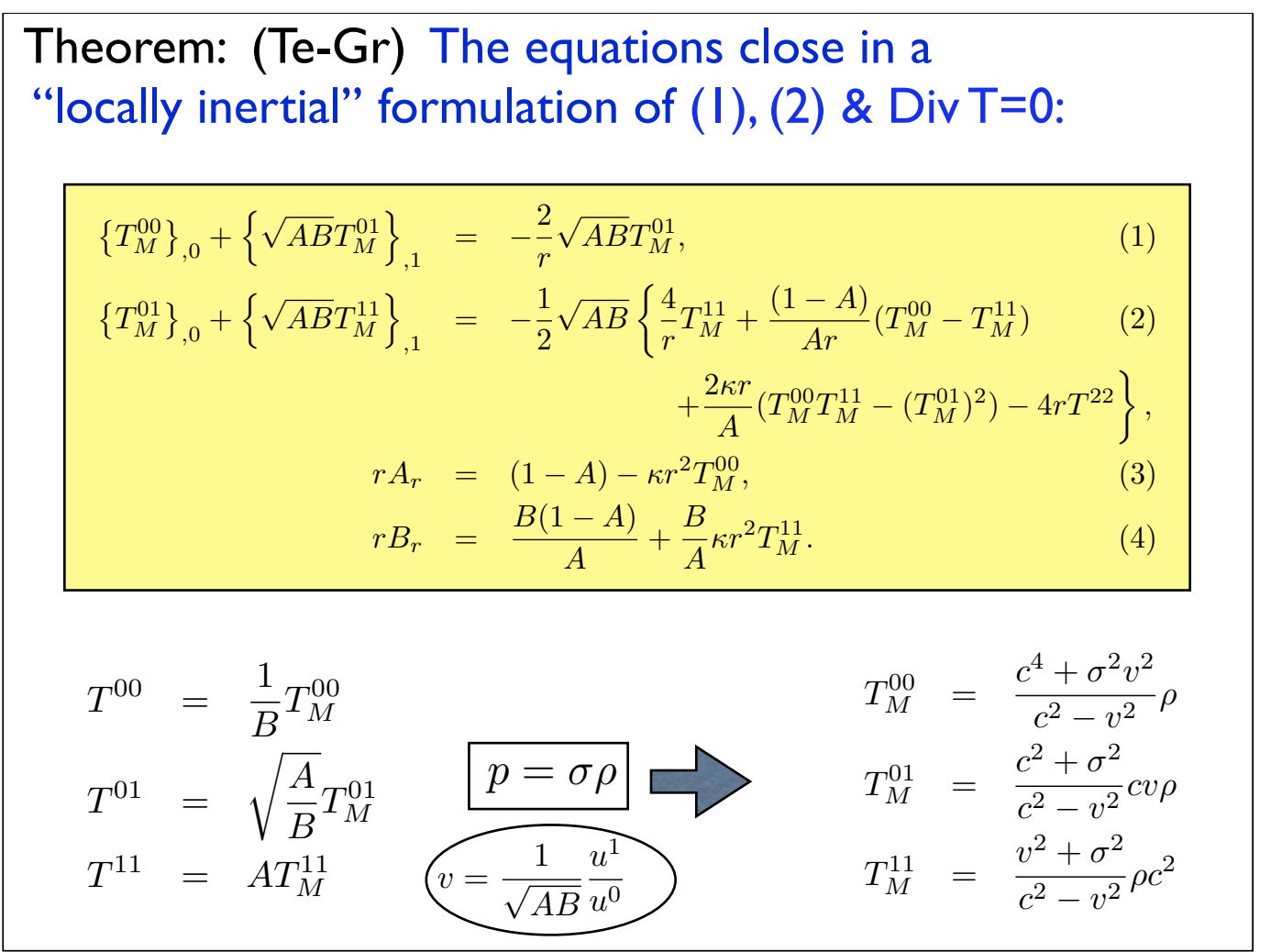




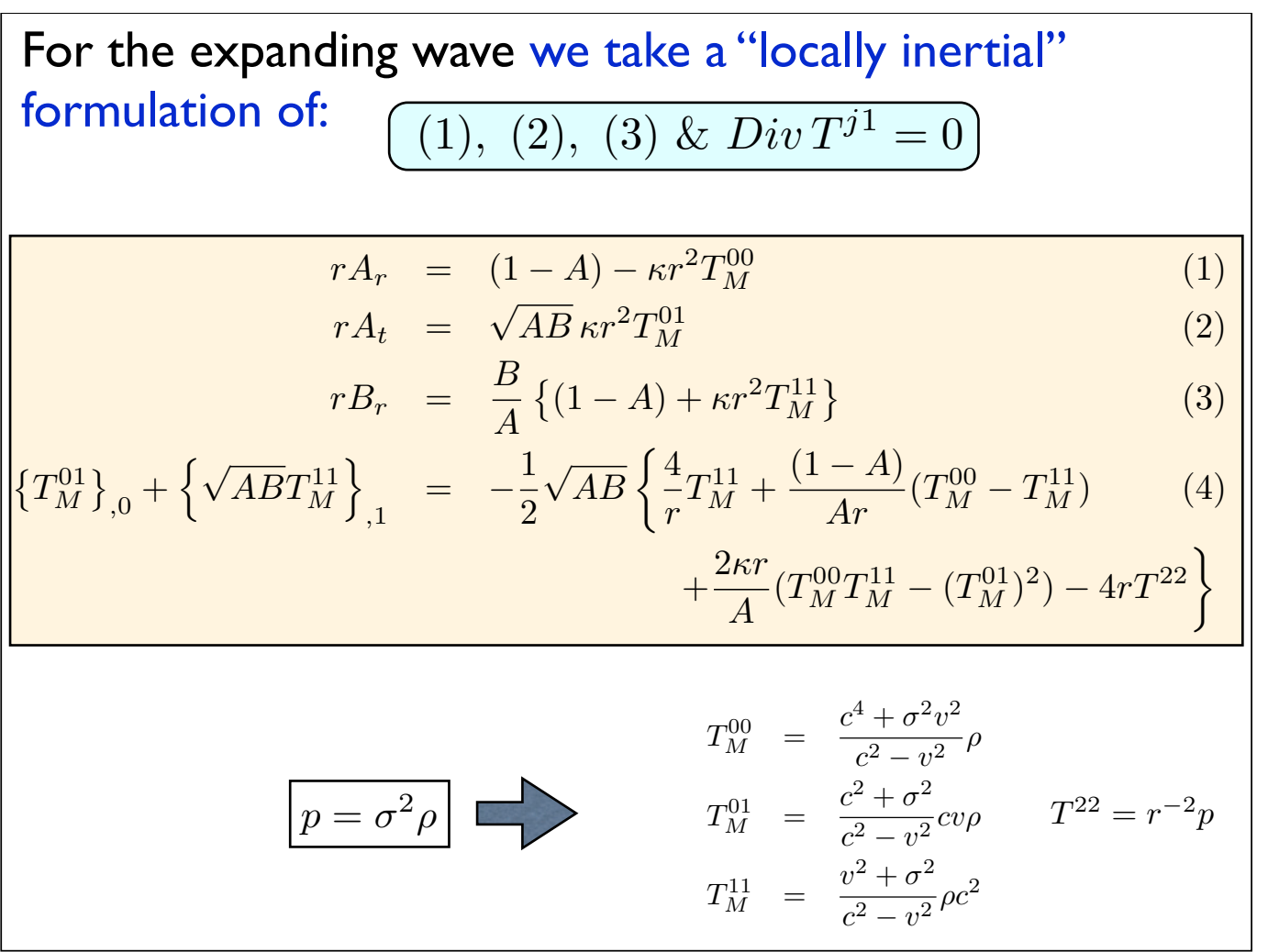


- Consider (I), (2) \& (3):

$$
\begin{aligned}
r A_{r} & =(1-A)-\kappa r^{2} T_{M}^{00} \\
r A_{t} & =\sqrt{A B} \kappa r^{2} T_{M}^{01} \\
r B_{r} & =\frac{B}{A}\left\{(1-A)+\kappa r^{2} T_{M}^{11}\right\}
\end{aligned}
$$

"The sources are linear in $r^{2} \rho "$

- Set:

$$
S^{i j} \equiv \kappa r^{2} T_{M}^{i j}
$$

So:

$$
S^{i j} \equiv \kappa w V^{i j}
$$

Where: $\quad \kappa w \equiv \frac{\kappa}{3} \rho r^{2}\left(1-v^{2}\right)^{-1}, \quad V^{i j} \equiv V^{i j}(v)$ 
OSubstituting $S^{i j}$, (I), (2) \& (3) become:

$$
\begin{aligned}
r A_{r} & =(1-A)-S^{00} \\
r A_{t} & =\sqrt{A B} S^{01} \\
r B_{r} & =\frac{B}{A}\left\{(1-A)+S^{11}\right\}
\end{aligned}
$$

- Now assume $A, B, S^{i j}$ depend only on $\xi=\frac{r}{t}$

$$
A=A(\xi), \quad B=B(\xi), \quad S^{i j}=S^{i j}(\xi)
$$

- Then $(1),(2) \&(3)$ all reduce to ODE's in $\xi !$ 
- (1), (2) \& (3) reduce to ODE's in $\xi ! !$

$$
\begin{aligned}
& \xi A_{\xi}=(1-A)-\kappa S^{00} \\
& \xi^{2} A_{\xi}=\sqrt{A B} \kappa S^{01} \\
& \xi B_{\xi}=\frac{B}{A}\left\{(1-A)+\kappa S^{11}\right\} \\
& S^{00}=\kappa r^{2} \rho \frac{c^{4}+\sigma^{2} v^{2}}{c^{2}-v^{2}}=\kappa\left\{\frac{r^{2} \rho}{3\left(1-v^{2}\right)}\right\}\left(3+v^{2}\right) \\
& S^{01}=\kappa r^{2} \rho \frac{c^{2}+\sigma^{2}}{c^{2}-v^{2}} c v=\kappa\left\{\frac{r^{2} \rho}{3\left(1-v^{2}\right)}\right\} 4 v \\
& S^{11}=\kappa r^{2} \rho \frac{\sigma^{2}+v^{2}}{c^{2}}=\kappa\{\begin{array}{c}
\begin{array}{c}
c=1 \\
\sigma^{2}=1 / 3
\end{array} \\
\frac{r^{2} \rho}{3\left(1-v^{2}\right)}
\end{array} \underbrace{\left(1+3 v^{2}\right)}_{\omega}
\end{aligned}
$$


(1), (2) \& (3) reduce to ODE's in $\xi \ldots$

$$
\begin{aligned}
\xi A_{\xi} & =(1-A)-\kappa S^{00} \\
\xi^{2} A_{\xi} & =\sqrt{A B} \kappa S^{01} \\
\xi B_{\xi} & =\frac{B}{A}\left\{(1-A)+\kappa S^{11}\right\}
\end{aligned}
$$

- Equations (I) \& (2) require the compatibility condition

$$
(1-A)-\kappa S^{00}=\frac{\sqrt{A B}}{\xi} \kappa S^{01}
$$

$$
\longleftrightarrow \quad \kappa w=\frac{(1-A) G}{\left(3+v^{2}\right) G-4 v} \quad G=\frac{\xi}{\sqrt{A B}}
$$


- Conclude: The compatibility condition

$$
\kappa w=\frac{(1-A) G}{\left(3+v^{2}\right) G-4 v}
$$

removes one equation and one variable $r^{2} \rho$

(Linearity in $\rho$, correct for $p=\frac{c^{2}}{3} \rho$, is crucial.)

- Said differently: once we get equations for

$$
(A, G, v)(\xi)
$$

we can use $(*)$ to solve for $r^{2} \rho$ 
- A similar reduction applies to Equation (4):

$\left\{T_{M}^{01}\right\}_{, 0}+\left\{\sqrt{A B} T_{M}^{11}\right\}_{, 1}=-\frac{1}{2} \sqrt{A B}\left\{\frac{4}{r} T_{M}^{11}+\frac{(1-A)}{A r}\left(T_{M}^{00}-T_{M}^{11}\right)\right.$ $\left.+\frac{2 \kappa r}{A}\left(T_{M}^{00} T_{M}^{11}-\left(T_{M}^{01}\right)^{2}\right)-4 r T^{22}\right\}$ 
- A similar reduction applies to Equation (4):

$\left\{T_{M}^{01}\right\}_{, 0}+\left\{\sqrt{A B} T_{M}^{11}\right\}_{, 1}=-\frac{1}{2} \sqrt{A B}\left\{\frac{4}{r} T_{M}^{11}+\frac{(1-A)}{A r}\left(T_{M}^{00}-T_{M}^{11}\right)\right.$

$$
\left.+\frac{2 \kappa r}{A}\left(T_{M}^{00} T_{M}^{11}-\left(T_{M}^{01}\right)^{2}\right)-4 r T^{22}\right\}
$$

- Multiplying through by $r^{3}$ and using $\left(^{*}\right)$ to eliminate $w$ and $w_{\xi}$ in favor of $v$ we obtain

(After considerable computation!) 
- A similar reduction applies to Equation (4):

$$
\begin{aligned}
\left\{T_{M}^{01}\right\}_{, 0}+\left\{\sqrt{A B} T_{M}^{11}\right\}_{, 1}=-\frac{1}{2} \sqrt{A B}\{ & \frac{4}{r} T_{M}^{11}+\frac{(1-A)}{A r}\left(T_{M}^{00}-T_{M}^{11}\right) \\
& \left.+\frac{2 \kappa r}{A}\left(T_{M}^{00} T_{M}^{11}-\left(T_{M}^{01}\right)^{2}\right)-4 r T^{22}\right\}
\end{aligned}
$$

- Multiplying through by $r^{3}$ and using $\left(^{*}\right)$ to eliminate $w$ and $w_{\xi}$ in favor of $v$ we obtain

(4)

$$
\begin{gathered}
\xi v_{\xi}=-\left(\frac{1-v^{2}}{2\{\cdot\}_{D}}\right)\left\{\left(3+v^{2}\right) G-4 v+\frac{4\left(\frac{1-A}{A}\right)\{\cdot\}_{N}}{\left(3+v^{2}\right) G-4 v}\right\} \\
\{\cdot\}_{N}=\left\{-2 v^{2}+2\left(3-v^{2}\right) v G-\left(3-v^{4}\right) G^{2}\right\} \\
\{\cdot\}_{D}=\left\{\left(3 v^{2}-1\right)-4 v G+\left(3-v^{2}\right) G^{2}\right\}
\end{gathered}
$$


- A similar reduction applies to Equation (4):

$$
\begin{aligned}
\left\{T_{M}^{01}\right\}_{, 0}+\left\{\sqrt{A B} T_{M}^{11}\right\}_{, 1}=-\frac{1}{2} \sqrt{A B}\{ & \frac{4}{r} T_{M}^{11}+\frac{(1-A)}{A r}\left(T_{M}^{00}-T_{M}^{11}\right) \\
& \left.+\frac{2 \kappa r}{A}\left(T_{M}^{00} T_{M}^{11}-\left(T_{M}^{01}\right)^{2}\right)-4 r T^{22}\right\}
\end{aligned}
$$

- Multiplying through by $r^{3}$ and using $\left(^{*}\right)$ to eliminate $w$ and $w_{\xi}$ in favor of $v$ we obtain

(4)

$$
\xi v_{\xi}=-\left(\frac{1-v^{2}}{2\{\cdot\}_{D}}\right)\left\{\left(3+v^{2}\right) G-4 v+\frac{4\left(\frac{1-A}{A}\right)\{\cdot\}_{N}}{\left(3+v^{2}\right) G-4 v}\right\}
$$

$G=\frac{\xi}{\sqrt{A B}}$

$$
\begin{aligned}
& \{\cdot\}_{N}=\left\{-2 v^{2}+2\left(3-v^{2}\right) v G-\left(3-v^{4}\right) G^{2}\right\} \\
& \{\cdot\}_{D}=\left\{\left(3 v^{2}-1\right)-4 v G+\left(3-v^{2}\right) G^{2}\right\}
\end{aligned}
$$


Conclude: $\quad(1)=(2),(3), \& \operatorname{Div}^{j 1}=0$

are Equivalent to:

$$
\begin{aligned}
\xi A_{\xi} & =-\left[\frac{4(1-A) v}{\left(3+v^{2}\right) G-4 v}\right] \\
(\mathrm{ODE}) \quad \xi G_{\xi} & =-G\left\{\left(\frac{1-A}{A}\right) \frac{2\left(1+v^{2}\right) G-4 v}{\left(3+v^{2}\right) G-4 v}-1\right\}
\end{aligned}
$$

$\xi v_{\xi}=-\left(\frac{1-v^{2}}{2\{\cdot\}_{D}}\right)\left\{\left(3+v^{2}\right) G-4 v+\frac{4\left(\frac{1-A}{A}\right)\{\cdot\}_{N}}{\left(3+v^{2}\right) G-4 v}\right\}$

$\{\cdot\}_{N}=\left\{-2 v^{2}+2\left(3-v^{2}\right) v G-\left(3-v^{4}\right) G^{2}\right\}$

$\{\cdot\}_{D}=\left\{\left(3 v^{2}-1\right)-4 v G+\left(3-v^{2}\right) G^{2}\right\}$

$$
G=\frac{\xi}{\sqrt{A B}} ; \xi=\frac{r}{t}
$$




$$
\begin{aligned}
\xi A_{\xi} & =-\left[\frac{4(1-A) v}{\left(3+v^{2}\right) G-4 v}\right] \\
\xi G_{\xi} & =-G\left\{\left(\frac{1-A}{A}\right) \frac{2\left(1+v^{2}\right) G-4 v}{\left(3+v^{2}\right) G-4 v}-1\right\} \\
\xi v_{\xi} & =-\left(\frac{1-v^{2}}{2\{\cdot\}_{D}}\right)\left\{\left(3+v^{2}\right) G-4 v+\frac{4\left(\frac{1-A}{A}\right)\{\cdot\}_{N}}{\left(3+v^{2}\right) G-4 v}\right\}
\end{aligned}
$$

A system of 3 ODE's analagous to the Oppenheimer-Volkoff Equations except they describe GR-Expansion Waves! 
Theorem: Assume that $A(\xi), G(\xi)$ and $v(\xi)$ solve ODE and use the constraint

$$
\kappa w \equiv \frac{r^{2} \rho}{3\left(1-v^{2}\right)}=\frac{(1-A) G}{\left(3+v^{2}\right) G-4 v}
$$

to define $\rho$

$$
\rho=\frac{1}{\kappa} \frac{3\left(1-v^{2}\right)(1-A) G}{\left(3+v^{2}\right) G-4 v} \frac{1}{\bar{r}^{2}} .
$$

Then the metric

$$
d s^{2}=-B(\xi) d \bar{t}^{2}+\frac{1}{A(\xi)} d \bar{r}^{2}+\bar{r}^{2} d \Omega^{2}
$$

solves the Einstein equations with

equation of state

$$
p=\rho c^{2} / 3 \text {. }
$$


- The Result: a system of three ODE's plus one constraint equivalent to the Einstein equations assuming $A, B, v$ and $r^{2} \rho$ depend only on $\xi=\frac{r}{t}$ :

$$
\begin{gathered}
\xi\left(\begin{array}{l}
A \\
E \\
v
\end{array}\right)_{\xi}=F\left(\begin{array}{l}
A \\
E \\
v
\end{array}\right) \\
\kappa w=\frac{1-A}{3+v^{2}-4 v E}
\end{gathered}
$$


- The equations for a three parameter family of GR-expansion waves

$\begin{aligned} \xi A_{\xi}= & -\left[\frac{4(1-A) v}{\left(3+v^{2}\right) G-4 v}\right] \\ \xi G_{\xi}= & -G\left\{\left(\frac{1-A}{A}\right) \frac{2\left(1+v^{2}\right) G-4 v}{\left(3+v^{2}\right) G-4 v}-1\right\} \\ \xi v_{\xi}= & -\left(\frac{1-v^{2}}{2\{\cdot\}_{D}}\right)\left\{\left(3+v^{2}\right) G-4 v+\frac{4\left(\frac{1-A}{A}\right)\{\cdot\}_{N}}{\left(3+v^{2}\right) G-4 v}\right\} \\ & \left\{\begin{array}{l}\text { (3) } \\ \end{array}\right. \\ & \{\cdot\}_{D}=\left\{\left(3 v^{2}-1\right)-4 v G+\left(3-v^{2}\right) G^{2}\right\} \\ \kappa w= & \frac{(1-A) G}{\left(3+v^{2}\right) G-4 v} \quad \text { (Compatibility Constraint) }\end{aligned}$


THEOREM: The equations are invariant under time-scaling

$$
t \rightarrow \alpha t \text {. }
$$

Except for this, solutions describe

\section{Distinct Spacetimes}

CONCLUDE: 3 -initial condts + I-scaling law

2-parameter family of GR-expansion waves 
Theorem: The coordinate mapping

$$
\begin{aligned}
& \bar{r}(t, r)=\sqrt{t} r \\
& \bar{t}(t, r)=\psi_{0}\left(1+\frac{r^{2}}{4}\right) t
\end{aligned}
$$

takes the $k=0, p=\frac{c^{2}}{3} \rho$ Freidmann universe

$$
d s^{2}=-d t^{2}+R(t)^{2}\left\{d r^{2}+r^{2} d \Omega^{2}\right\}
$$

to I-point in this 2-parameter family of

GR-expansion waves 


\section{Proof: Coordinate mapping IMPLIES:}

$$
A=1-v^{2}, \quad E=\frac{1}{\psi_{0} \xi}, \quad \xi=\frac{2 v}{\psi_{0}\left(1+v^{2}\right)}, \quad v_{\xi}=\frac{\psi_{0}\left(1+v^{2}\right)^{2}}{2\left(1-v^{2}\right)}
$$

Plug in and check:

$$
\begin{aligned}
& \xi A_{\xi}=-\left[\frac{4(1-A) v}{\left(3+v^{2}\right) G-4 v}\right] \\
& \xi G_{\xi}=-G\left\{\left(\frac{1-A}{A}\right) \frac{2\left(1+v^{2}\right) G-4 v}{\left(3+v^{2}\right) G-4 v}-1\right\} \\
& \xi v_{\xi}=-\left(\frac{1-v^{2}}{2\{\cdot\}_{D}}\right)\left\{\left(3+v^{2}\right) G-4 v+\frac{4\left(\frac{1-A}{A}\right)\{\cdot\}_{N}}{\left(3+v^{2}\right) G-4 v}\right\} \\
& \{\cdot\}_{N}=\left\{-2 v^{2}+2\left(3-v^{2}\right) v G-\left(3-v^{4}\right) G^{2}\right\} \\
& \{\cdot\}_{D}=\left\{\left(3 v^{2}-1\right)-4 v G+\left(3-v^{2}\right) G^{2}\right\} \\
& \kappa w=\frac{(1-A) G}{\left(3+v^{2}\right) G-4 v} \quad(\text { Compatibility Constraint })
\end{aligned}
$$

\section{"A surprisingly long calculation!"}


Technicalities (for the $v_{\xi}$-equation):

$$
0=\left(-V^{01}+E V^{11}\right) \xi \frac{w_{\xi}}{w}+\left(-4+2 E V^{01}\right) \xi v_{\xi}+\xi \frac{A_{\xi}}{A} V^{01}
$$

$$
+E \xi \frac{B_{\xi}}{B} V^{01}\left(V^{00}+V^{11}\right)-2 E V^{22}
$$

Using identities that hold for standard Model as expressed in SSC's, we can reduce this sum to: 


$$
\begin{aligned}
& \text { (1) }=\left(-4 v+\frac{1+v^{2}}{2 v}\left(1+3 v^{2}\right)\right) \frac{2\left(1+v^{2}\right)}{\left(1-v^{2}\right)^{2}} \\
& + \\
& \text { (2) }=2\left(-2+3 \frac{1+v^{2}}{2}\right) \frac{v\left(1+v^{2}\right)}{\left(1-v^{2}\right)} \\
& + \\
& \text { (3) }=-4 \frac{2\left(1+v^{2}\right)}{\left(1-v^{2}\right)^{2}} v^{3} \\
& \text { (4) }=4 \frac{\left(1+v^{2}\right)^{3}}{2 v\left(1-v^{2}\right)^{2}} \\
& + \\
& \text { (5) }=-2 \frac{1+v^{2}}{2 v}\left(1-v^{2}\right) \\
& \text { The sum is equal to zero! }
\end{aligned}
$$


Conclude: The standard model of cosmology after inflation represents one solution of our ODE's corresponding to one initial condition... 


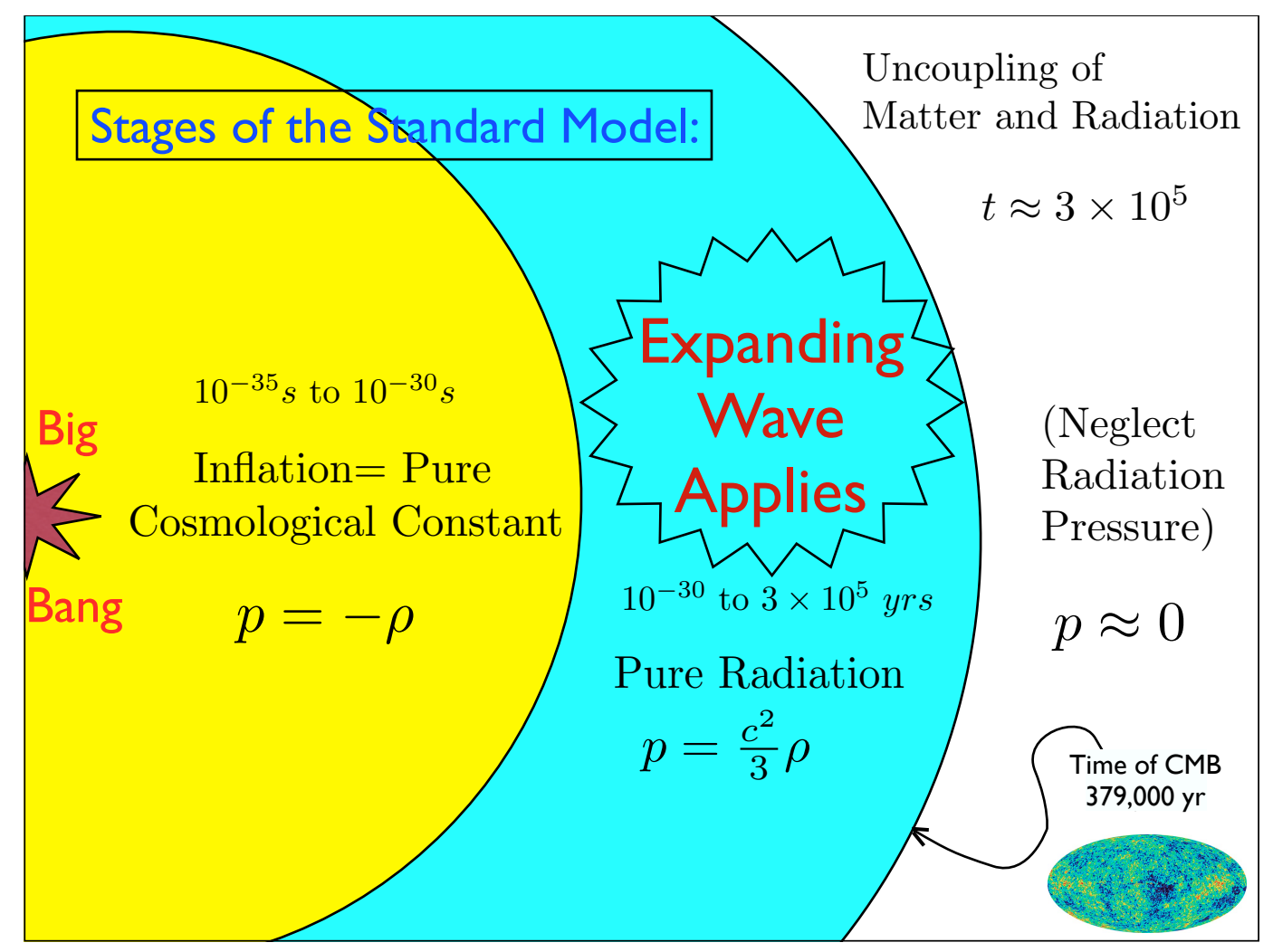


Since the standard model represents I-point in a

2-parameter family,

we look for

leading order corrections to the standard model determined from the nearby

GR-expansion waves 
Linearizing about the center $\xi=0$ :

- One eigen-family tends to infinity as $\xi \rightarrow 0$

- Two eigen-solutions stay finite as $\xi \rightarrow 0$ and:

$$
A \rightarrow 1, B \rightarrow 1, v \rightarrow 0
$$

(One parameter is the scaling law...)

- Conclude: There is a smooth I-parameter family of distinct spacetimes that extend the standard model! 


\section{Let}

$$
\begin{aligned}
& \psi_{0} \equiv \text { Scaling Parameter } \\
& a \equiv \text { Acceleration Parameter }
\end{aligned}
$$

and let

$$
v \equiv v_{1}(\xi)
$$

denote the velocity profile for the FRW standard model... 
The following Theorem shows:

"Nearby solutions stay surprising close to FRW..." 
Theorem: There exist positive constants $\left(\psi_{0}, a\right)$ such that the following estimates hold near $\xi=0$.

$$
\begin{aligned}
v(\xi) & =v_{1}(\xi)+\frac{\left(1-a^{2}\right)}{8} \psi_{0}^{3} \xi^{3}+O(1)|a-1| \xi^{4} \\
A(\xi) & =1-\frac{a^{2} \psi_{0}^{2}}{4} \xi^{2}+O(1)|a-1| \xi^{4} \\
G(\xi) & =\psi_{0} \xi+O(1)|a-1| \xi^{5} \\
\sqrt{A B} & =\frac{1}{\psi_{0}}+O(1)|a-1| \xi^{4}
\end{aligned}
$$


Theorem 1: To leading order in $\xi$, the 1-parameter family that extends the standard model of cosmology is given in SSC's by

$$
d s^{2}=-\frac{d \bar{t}^{2}}{\psi_{0}^{2}\left(1-\frac{a^{2} \psi_{0}^{2} \xi^{2}}{4}\right)}+\frac{d \bar{r}^{2}}{\left(1-\frac{a^{2} \psi_{0}^{2} \xi^{2}}{4}\right)}+\bar{r}^{2} \Omega^{2}
$$

$$
\begin{gathered}
v=\frac{\psi_{0}}{2} \xi \\
\xi=\frac{\bar{r}}{t}
\end{gathered}
$$

$(a=1) \equiv$ Standard Model 
Theorem 1: To leading order in $\xi$, the 1-parameter family that extends the standard model of cosmology is given in SSC's by

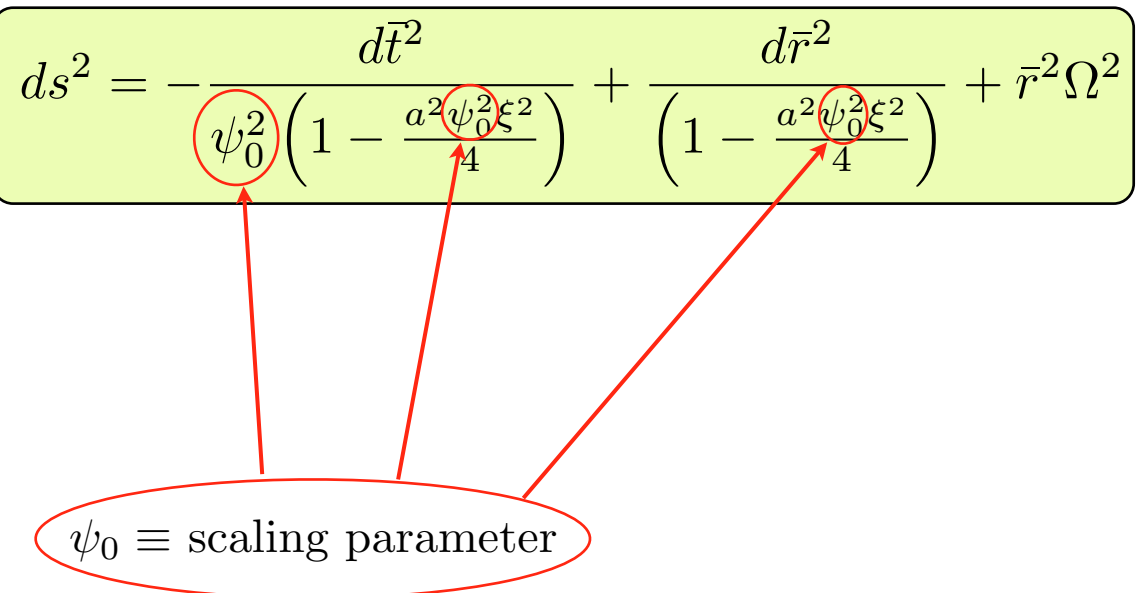




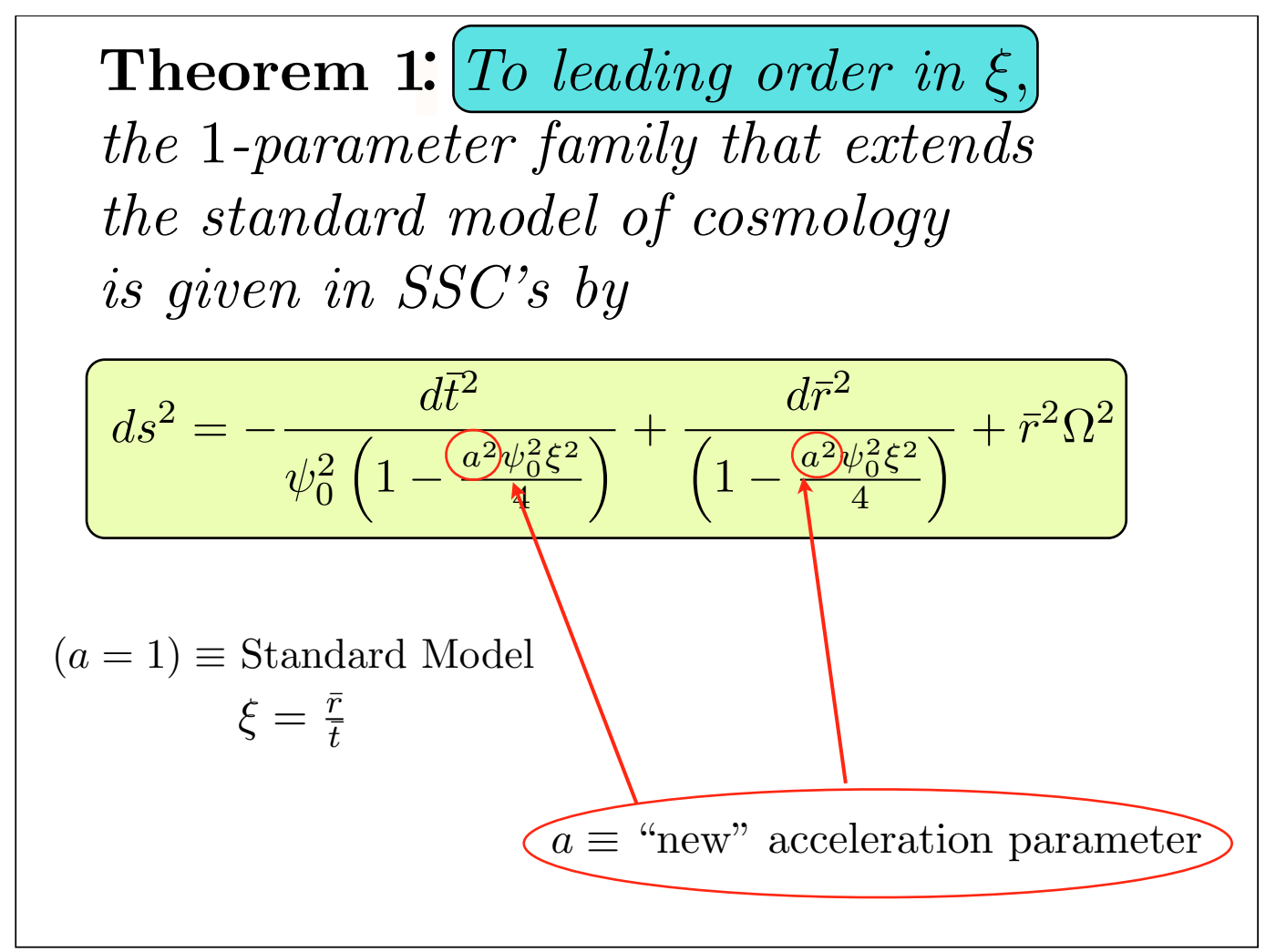


Theorem 1: To leading order in $\xi$, the 1-parameter family that extends the standard model of cosmology is given in SSC's by

$$
d s^{2}=-\frac{d \bar{t}^{2}}{\psi_{0}^{2}\left(1-\frac{a^{2} \psi_{0}^{2} \xi^{2}}{4}\right)}+\frac{d \bar{r}^{2}}{\left(1-\frac{a^{2} \psi_{0}^{2} \xi^{2}}{4}\right)}+\bar{r}^{2} \Omega^{2}
$$

$$
v=\frac{\psi_{0}}{2} \xi \quad \text { The velocity is }
$$
independent of $a$ ! 


\begin{tabular}{|c|}
\hline $\begin{array}{c}\text { Since the velocity field is } \approx \\
\text { independent of "a", it follows that the } \\
\underline{\text { inverse mapping from }} \\
\text { Standard Model to SSC's } \\
\text { provides }\end{array}$ \\
\hline $\begin{array}{c}\text { a co-moving coordinate system } \\
\text { to leading order in } \xi\end{array}$ \\
\hline $\begin{array}{l}\bar{r}(t, r)=\sqrt{t} r \\
\bar{t}(t, r)=\psi_{0}\left(1+\frac{r^{2}}{4}\right) t\end{array}$ \\
\hline
\end{tabular}




Back in Friedmann coordinates,
the metric "corrections" depend only on
\[ =\frac{\bar{r}}{t} \]
$\approx$ "Distance from Center to Hubble Length"
$0 \leq \zeta<<1$




Back in Friedmann coordinates,
the metric "corrections" depend only on
$\zeta=\frac{\bar{r}}{t}$
$\zeta \equiv \frac{\bar{r}}{c t} \approx \frac{R(t) r}{(c / H)} \approx \frac{\text { Dist }}{H u b b l e \text { Length }}$
$\approx$ "Fractional Distance From Center to Hubble Length"
$0 \leq \zeta<<1$




\section{The coord. mapping:}

$$
\begin{gathered}
d s^{2}=-F_{a}(\zeta)^{2} d t^{2}+F_{a}(\zeta)^{2} t d r^{2}+\bar{r}^{2} d \Omega^{2} \\
F_{a}(\zeta)^{2}=1+\left(a^{2}-1\right) \frac{\zeta^{2}}{4}+O\left(|a-1| \zeta^{4}\right) \\
v=O\left(|1-a| t^{\frac{1}{2}} \zeta^{3}\right)
\end{gathered}
$$

$\zeta=\frac{\bar{r}}{t} \approx$ "Distance from Center to Hubble Length"

$$
0 \leq \zeta<<1
$$




\section{C.f. Standard Model:}

$$
d s^{2}=-F_{a}(\zeta)^{2} d t^{2}+\underbrace{F_{a}(\zeta)^{2} t}_{R_{a}(t, \zeta)^{2}} d r^{2}+\bar{r}^{2} d \Omega^{2}
$$

Define the "Hubble Constant": $\quad H_{a}(t, \zeta)=\frac{1}{R} \frac{\partial}{\partial t} R$ Then:

$$
H_{a}(t, \zeta)=\frac{1}{2 t}\left\{1-\frac{3}{8}\left(a^{2}-1\right) \zeta^{2}+O\left(\left|a^{2}-1\right| \zeta^{4}\right)\right\}
$$

C.f. Standard Model: $\quad H_{1}=\frac{1}{2 t}$ 
Conclude: an observer at the center would measure a fractional correction to the Hubble constant on the order of...

$$
\Delta_{a} \equiv \frac{H_{a}-H}{H}=\frac{3}{8}\left(1-a^{2}\right) \zeta^{2}+O\left(\left|a^{2}-1\right| \zeta^{4}\right)
$$

$$
\zeta \equiv \frac{\bar{r}}{c t} \approx \frac{\bar{r}}{(c / H)} \approx \frac{\text { Dist }}{\text { Hubble Length }}
$$

$\approx$ "Fractional Distance from Center to Furthest Visible Objects" 
Moreover: using co-moving coordinates, we can calculate the leading order correction to the redshift vs luminosity relation as measured by an observer at the center of the spacetime: 


\section{LET:}

$$
d_{\ell} \equiv \text { Luminosity Distance }=\left(\frac{L}{4 \pi \ell}\right)^{1 / 2}
$$




\section{LET:}

$$
\begin{aligned}
& d_{\ell} \equiv \text { Luminosity Distance }=\left(\frac{L}{4 \pi \ell}\right)^{1 / 2} \\
& L=\text { Absolute Luminosity }=\frac{\text { Energy Emitted by Source }}{\text { Time }}
\end{aligned}
$$




\section{LET:}

$$
\begin{aligned}
& d_{\ell} \equiv \text { Luminosity Distance }=\left(\frac{L}{4 \pi \ell}\right)^{1 / 2} \\
& L=\text { Absolute Luminosity }=\frac{\text { Energy Emitted by Source }}{\text { Time }}
\end{aligned}
$$

$\ell \equiv$ Apparent Luminosity $=\frac{\text { Power Recieved }}{\text { Area }}$ 


\section{LET:}

$$
\begin{aligned}
& d_{\ell} \equiv \text { Luminosity Distance }=\left(\frac{L}{4 \pi \ell}\right)^{1 / 2} \\
& L=\text { Absolute Luminosity }=\frac{\text { Energy Emitted by Source }}{\text { Time }}
\end{aligned}
$$$$
\ell \equiv \text { Apparent Luminosity }=\frac{\text { Power Recieved }}{\text { Area }}
$$

$$
z=\frac{\lambda_{0}}{\lambda_{e}}-1=\text { Redshift Factor }
$$


THEN:

A calculation implies... 
The redshift vs luminosity relation as measured by an observer at the center of the spacetime is given by:

$d_{\ell}=2 t_{0} z\left\{1+\frac{a^{2}-1}{2} z+\cdots\right.$ 
The redshift vs luminosity relation as measured by an observer at the center of the spacetime is given by:

$d_{\ell}=2 t_{0} z\left\{1+\frac{a^{2}-1}{2} z\right.$

$+H . O . T$

...Quadratic correction quoted in PNAS... 
The redshift vs luminosity relation as measured by an observer at the center of the spacetime is given by:

$d_{\ell}=2 t_{0} z\left\{1+\frac{a^{2}-1}{2} z+\frac{\left(a^{2}-1\right)\left(6 a^{2}+13\right)}{6} z^{2}\right\}$

+ H.O.T 
The redshift vs luminosity relation as measured by an observer at the center of the spacetime is given by:

$$
\begin{array}{r}
d_{\ell}=2 t_{0} z\left\{1+\frac{a^{2}-1}{2} z+\frac{\left(a^{2}-1\right)\left(6 a^{2}+13\right)}{6} z^{2}\right\} \\
+ \text { H.O.T }
\end{array}
$$

...Cubic correction MUCH harder... (to appear SM/TE) 
The redshift vs luminosity relation as measured by an observer at the center of the spacetime is given by:

$$
\begin{array}{r}
d_{\ell}=2 t_{0} z\left\{1+\frac{a^{2}-1}{2} z+\frac{\left(a^{2}-1\right)\left(6 a^{2}+13\right)}{6} z^{2}\right\} \\
+ \text { H.O.T }
\end{array}
$$

(The calculation is nontrivial, and relies on simplifying features of the spacetime metric of the nearby expanding wave solutions...) 


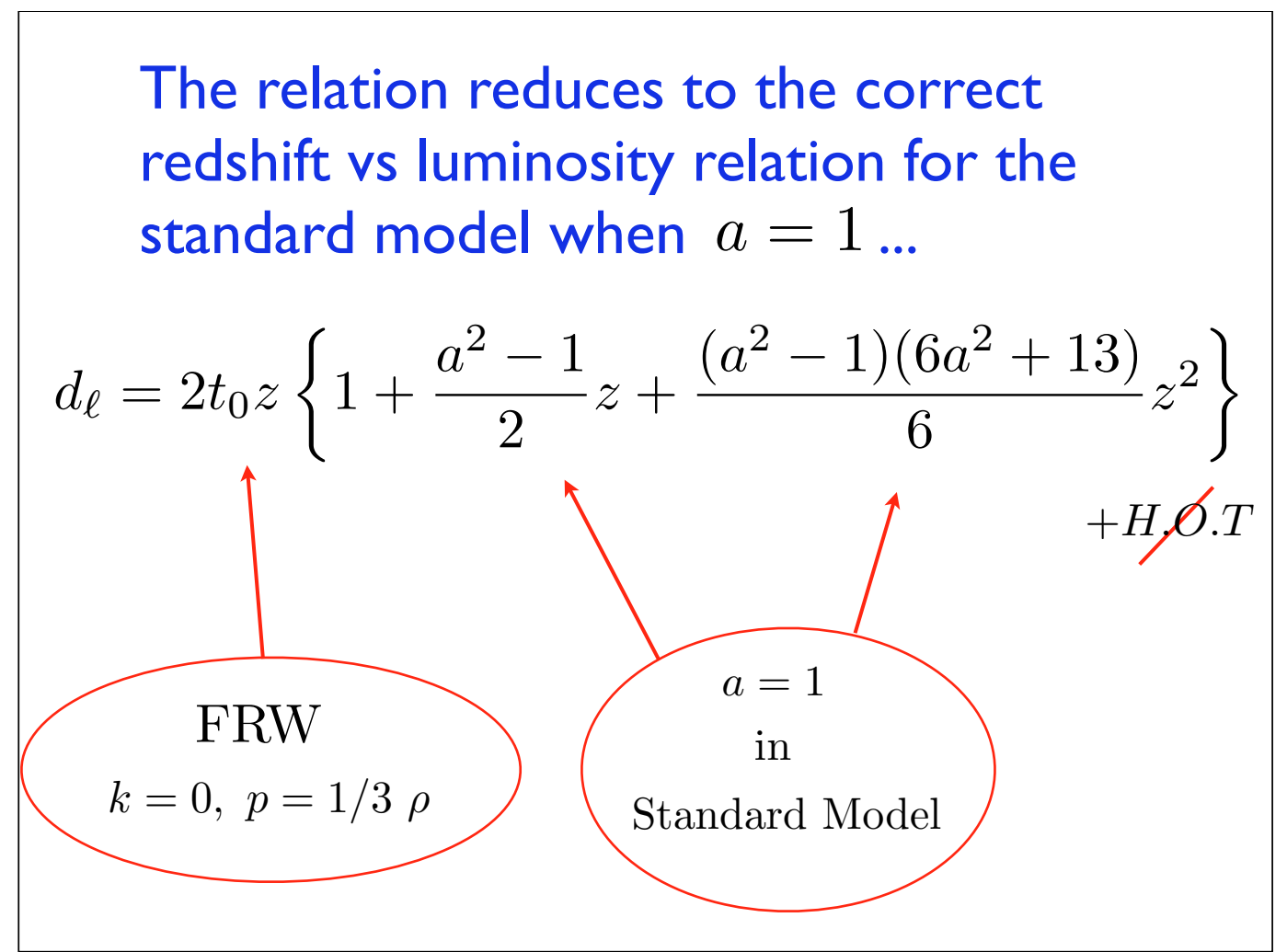




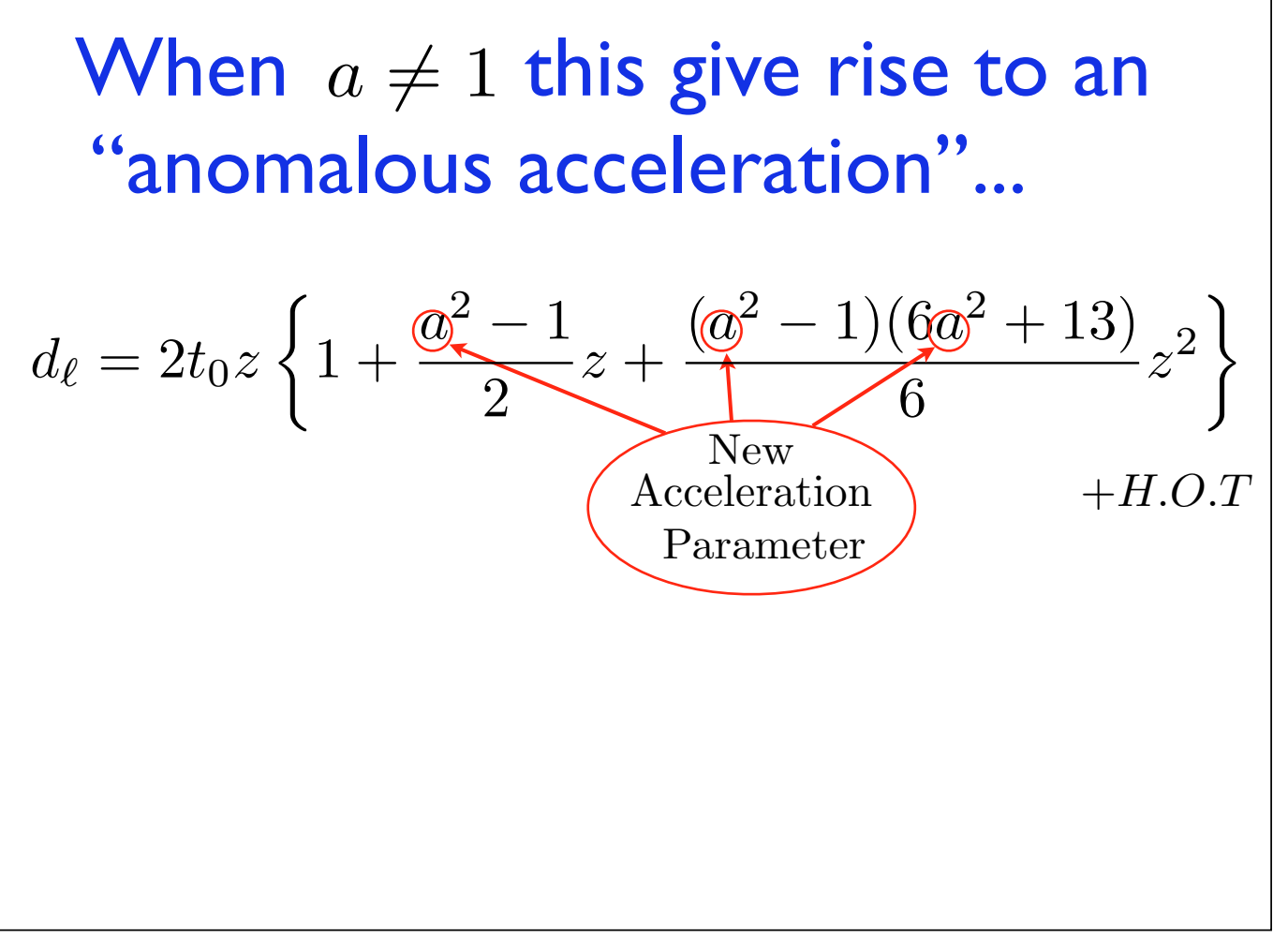


When $a \neq 1$ this give rise to an "anomalous acceleration"...

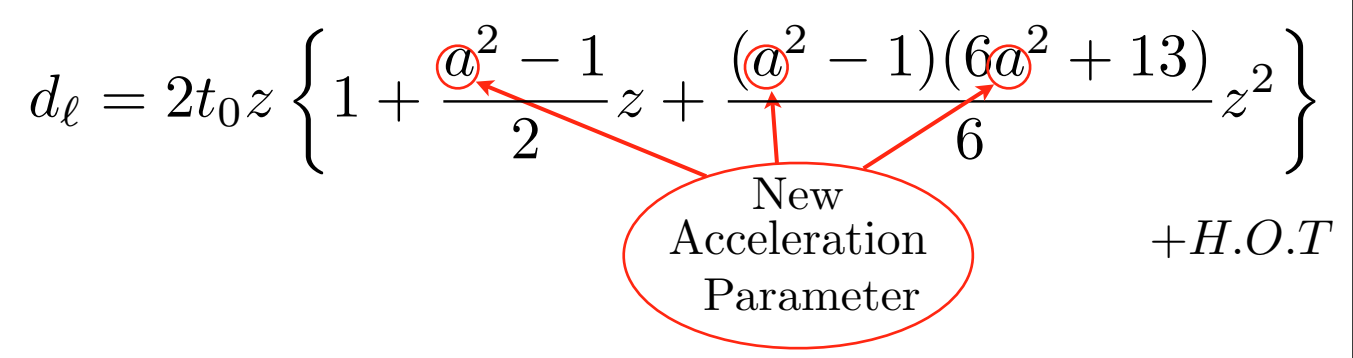

...a rigorous observable and quantifiable correction to the redshift vs luminosity relation... 


\section{After the radiation phase:}

The redshift vs luminosity relation evolves continuously with time

Therefore... 
We conclude (by continuity) corrections to the redshift vs luminosity relation observed after the radiation phase of the Big Bang can be accounted for, at the leading order quadratic level, by adjustment of the free parameter "a". 
The next order correction is a

VERIFIABLE PREDICTION of the model!!

(Work in progress) 
A different coord. mapping casts new metric in a different light: 
A different coord. mapping casts new metric in a different light:

$$
\begin{aligned}
\bar{r}(t, r) & =\frac{t^{a}}{2} r \\
\bar{t}(t, r) & =\psi_{0}\left(1+\frac{a^{2} \zeta^{2}}{4}\right) t
\end{aligned}
$$




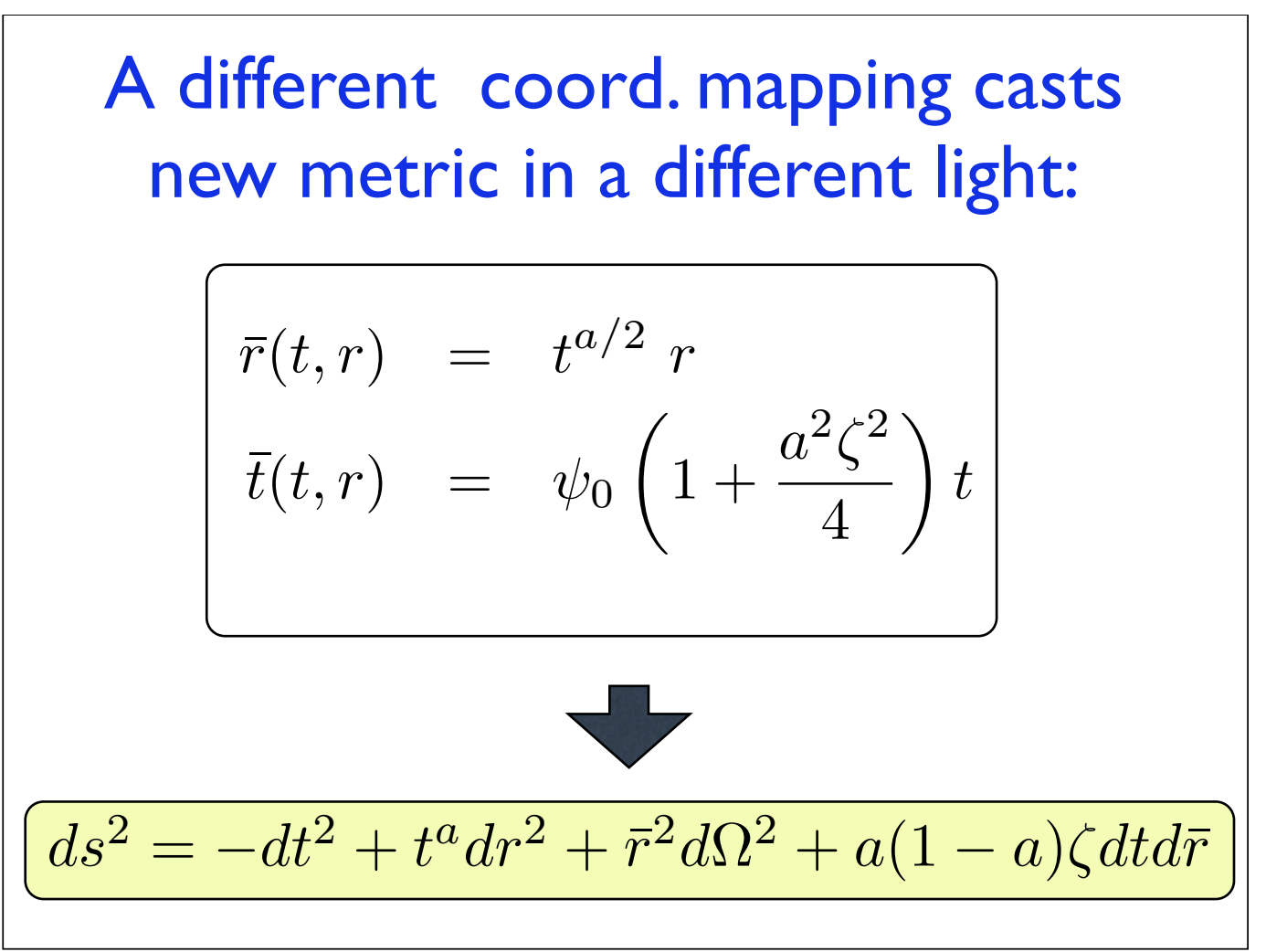


Conclude: in special non-comoving coords:

$d s^{2}=-d t^{2}+t^{a} d r^{2}+\bar{r}^{2} d \Omega^{2}+a(1-a) \zeta d t d \bar{r}$

"Looks like standard model with a small correction to the expansion rate, and a small corrective mixed term"

Error: $\quad O\left(|t+(a-1)| \zeta^{3}\right)$ 


"In Fact: In these coordinates...
metric is
exactly flat 3 -space
at each fixed
$\mathrm{t}=$ const
...just like the standard model..."
$d s^{2}=-d t^{2}+t^{a}\left\{d r^{2}+r^{2} d \Omega^{2}\right\}+a(1-a) \zeta d t d \bar{r}$


A "Conservation Law" Scenario of the Big Bang w/o Cosmological Constant:

- Conservation Laws Decay to Non-interacting Time-Asymptotic Wave Patterns.

- After inflation, Universe is nearly flat, but due to errors, it decays by the nonlinearities of the radiation phase $a \neq 1$ to a nearby non-interacting expansion wave

- We happen to be near the center of expansion, so looking outward, we observe a critical FRW

with a small correction 
The Lesson of Conservation Laws...

"Expansion waves and shock waves are fundamental to conservation laws, because even when dissipative terms are neglected, shock-wave dissipation by itself causes non-interacting wave patterns to emerge from interactive solutions" 
"I.e. The one fact most certain about the Standard Model is an early hot dense epoch in which all energy was radiation..." 
"...one might reasonably conjecture that decay to a non-interacting expanding wave might have occured (locally??)

during the radiation phase due to the large nonlinearities associated with the large sound speed when $p=\frac{c^{2}}{3} \rho$." 


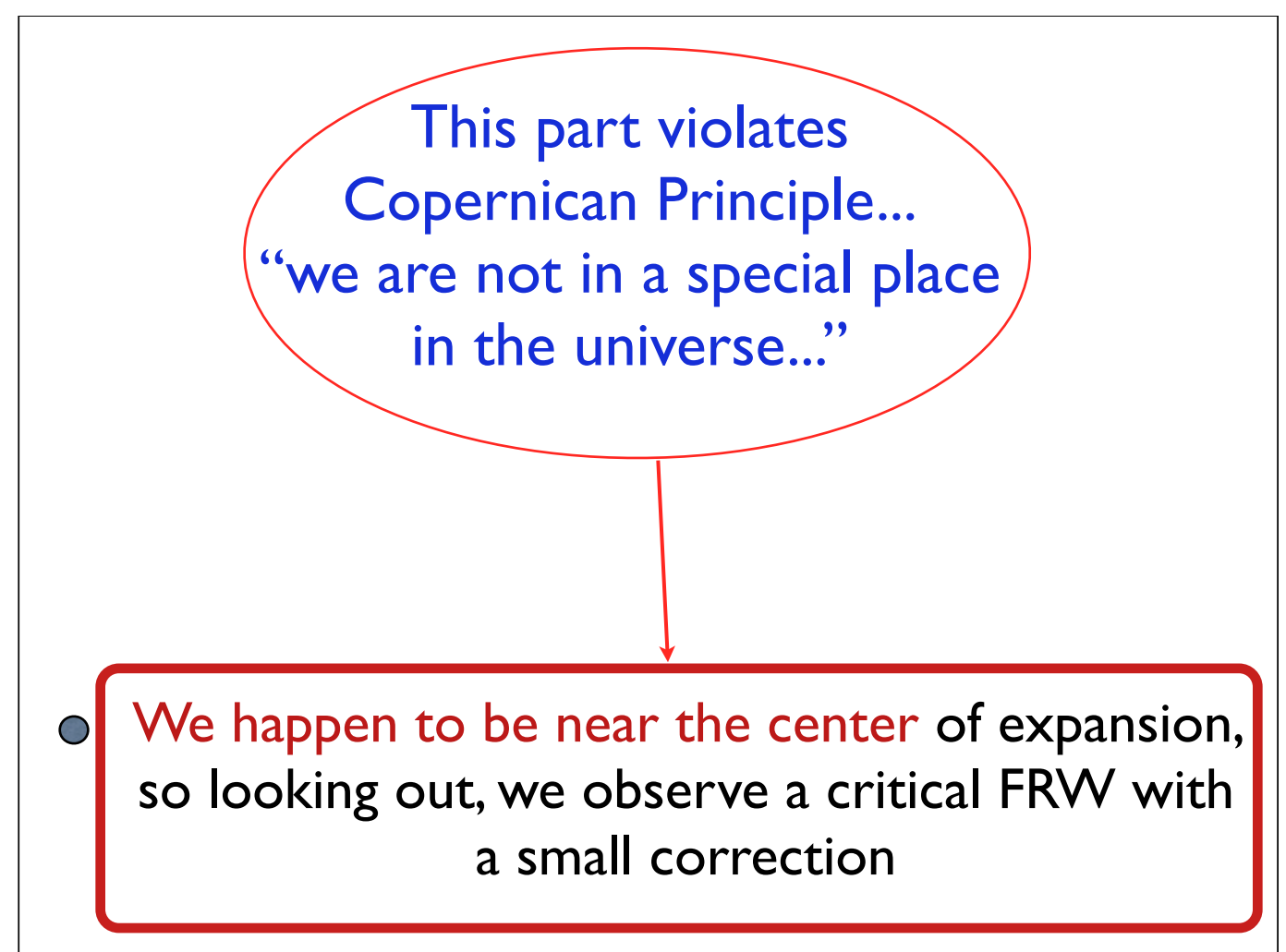


To make a testable prediction, we need to get the corrections at $t=379,000$ yrs, propagate errors with$$
p=0
$$$$
\text { to present time, }
$$$$
\text { and }
$$$$
\text { look for the best fit. }
$$ 


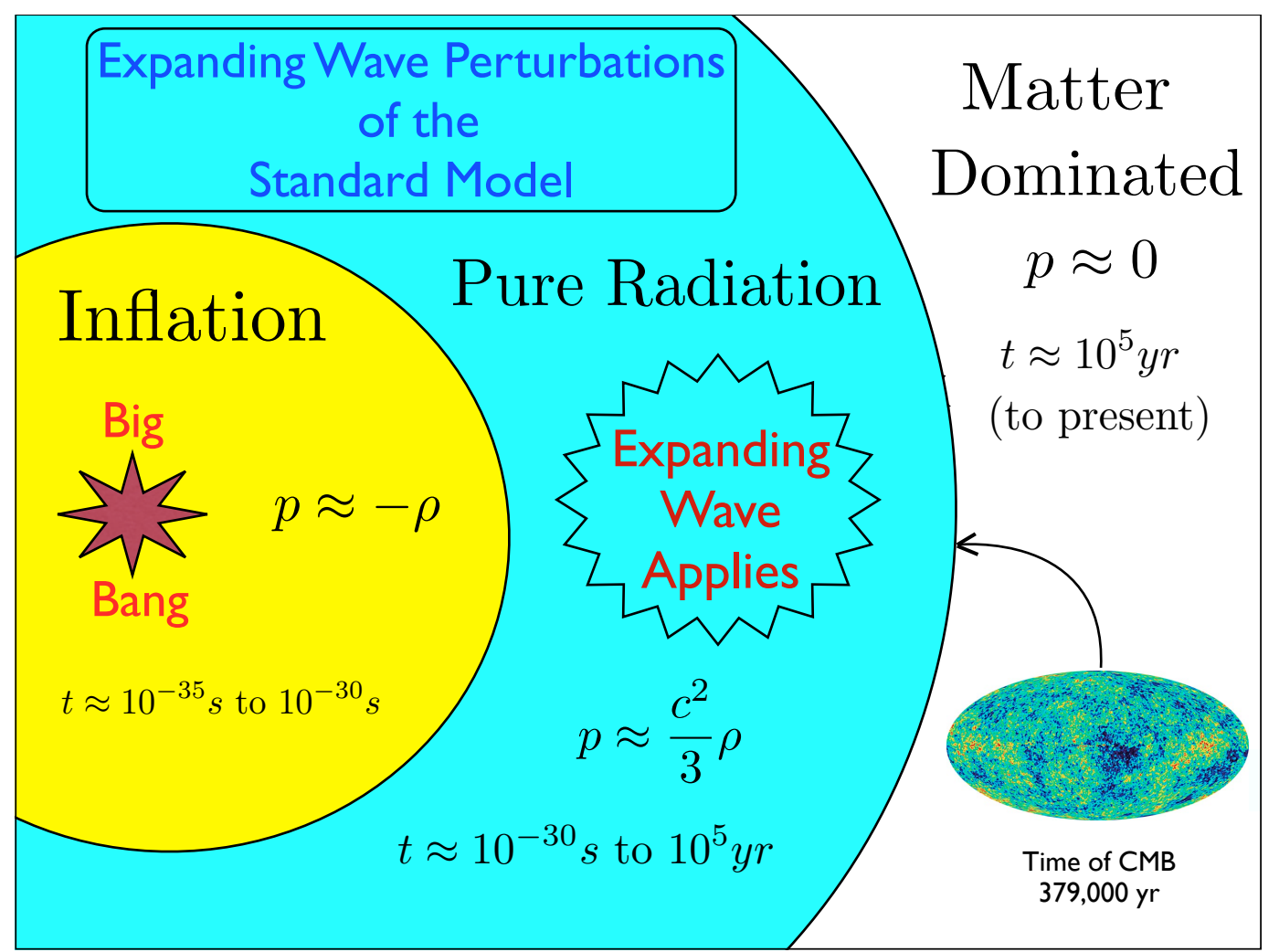


Note: The expansion wave may not propagate as self-similar

AFTER the radiation phase! 
We Like:

- This correction to the Hubble Constant is not put in "Ad Hoc"...

- It is derived from first principles starting from a theory of

$$
\text { Expansion Waves }
$$


We Wonder:

- What scale might such expanding waves exist on...?

- Is there an inconsistency with WMAP Data...?

- Can this be accounted for in some inflationary scenario...? 
Final Comment: These expanding waves near $k=0$ FRW represent a sort of "instability" in the Standard Model...

Thus: Even if they do not account for the anomalous acceleration...

One Has to Wonder why the Universe would choose $a=I, k=0, F R W$, and not one of these nearby non-interacting

Expansion Waves? 


\begin{tabular}{|c|c|c|}
\hline SciTech & & \\
\hline SCITECH HOME & \multirow{2}{*}{\multicolumn{2}{|c|}{$\begin{array}{l}\text { FOXNEWS.COM HOME > SCITECH } \\
\text { Scientists: Earth May Exist in Giant Cosmic Bubble }\end{array}$}} \\
\hline ARCHAEOLOGY & & \\
\hline CYBERSECURITY & \multirow{2}{*}{\multicolumn{2}{|c|}{$\begin{array}{l}\text { Wednesday, October } 01,2008 \\
\text { By Clara Moskowitz } \\
\text { SPACE. }\end{array}$}} \\
\hline $\begin{array}{l}\text { EVOLUTION AND } \\
\text { PALEONTOLOGY }\end{array}$ & & \\
\hline NATURAL & & E-Mail $\mid$ Print \\
\hline $\begin{array}{l}\text { PATENTS AND } \\
\text { INNOVATION }\end{array}$ & 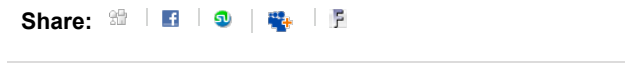 & \\
\hline $\begin{array}{l}\text { PERSONAL } \\
\text { TECHNOLOGY } \\
\text { SPACE }\end{array}$ & & $\begin{array}{l}\text { If the notion of dark energy } \\
\text { sounds improbable, get ready for } \\
\text { an even more outlandish } \\
\text { suggestion. }\end{array}$ \\
\hline $\begin{array}{l}\text { SPACE } \\
\text { VIDEO GAMING }\end{array}$ & & Earth may be trapped in an abnorm $z$ \\
\hline NEWS ARCHIVE & & $\begin{array}{l}\text { bubble of Space-time that is } \\
\text { particularly devoid of matter. }\end{array}$ \\
\hline $\begin{array}{l}\text { HOT TOPICS } \\
\text { - FOX NEWS } \\
\text { ELECIION } \\
\text { COVERAGE } \\
\text { - CELEBRITY }\end{array}$ & & $\begin{array}{l}\text { Scientists say this condition could } \\
\text { account for the apparent acceleratior } \\
\text { of the universe's expansion, for } \\
\text { which dark energy currently is the } \\
\text { leading explanation. }\end{array}$ \\
\hline $\begin{array}{l}- \text { FOX } \\
\text { MOVIETONE } \\
\text { NEWS }\end{array}$ & & $\begin{array}{l}\text { Dark energy is the name given to the } \\
\text { hypothetical force that could be } \\
\text { drawing all the stuff in the universe }\end{array}$ \\
\hline SECTION MAP & NASA/CXC/MIT/UMass & outward at an ever-increasing rate. \\
\hline SEE MORE & & $\begin{array}{l}\text { Current thinking is that } 74 \text { percent of } \\
\text { the universe could be made up of } \\
\text { this exotic dark energy, with another }\end{array}$ \\
\hline
\end{tabular}









\section{DARK ENERGY}

Maybe not

Really Exist?

The obset vations thet ied astronomers to

expanation: that our galaxy lies at the center

of a giant cosmic void

By Timothy Clifton and

Pedro G. Ferreirg

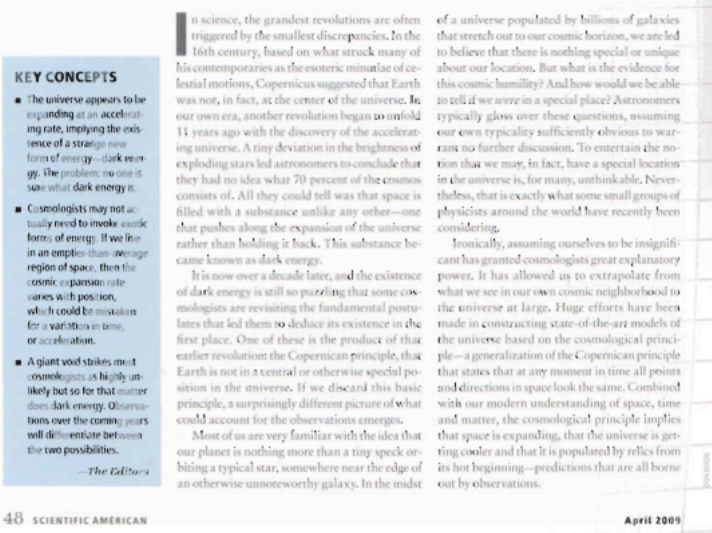




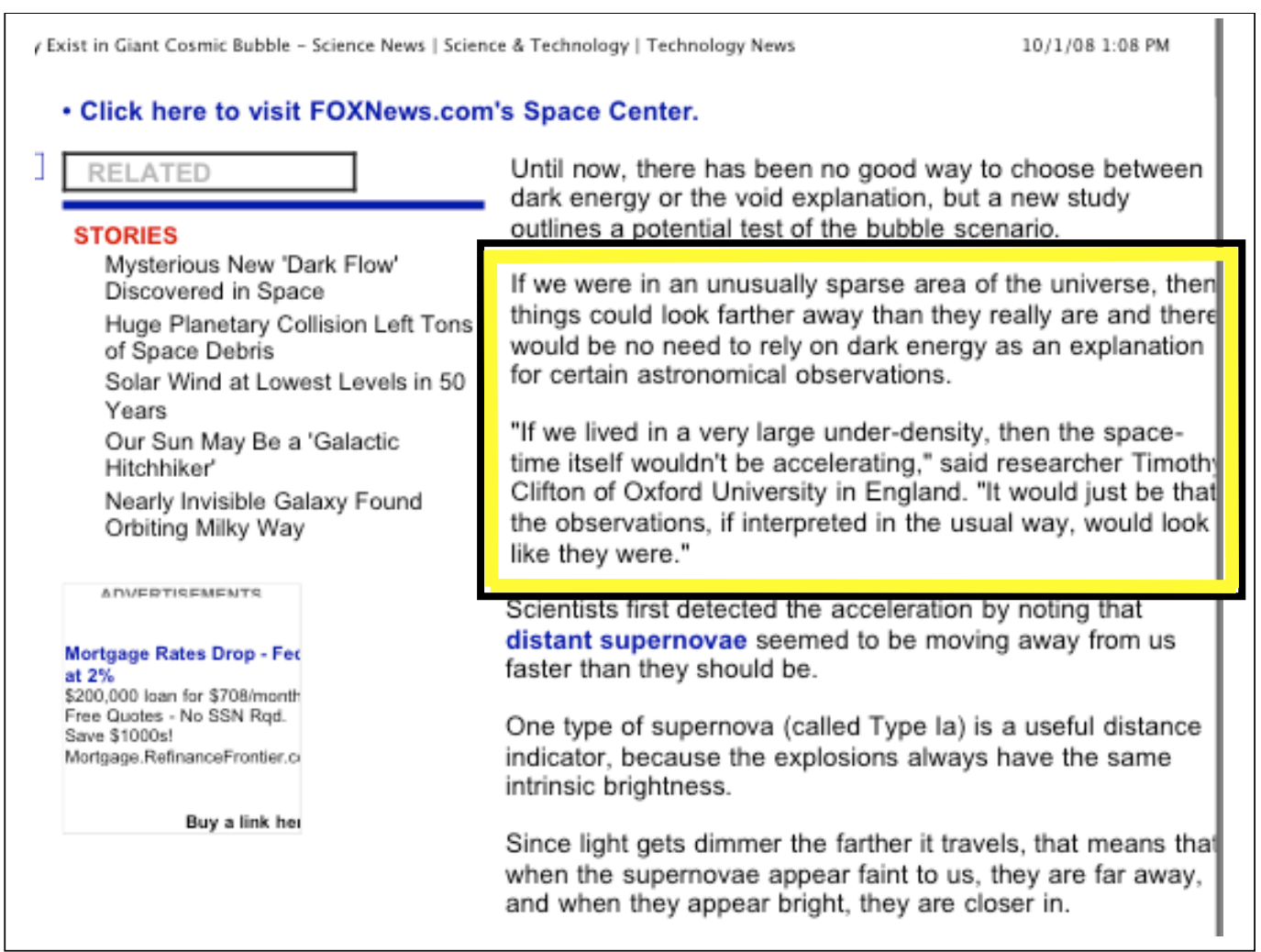


Until now, there has been no good way to choose between dark energy or the void explanation, but a new study

outlines a potential test of the bubble scenario.

If we were in an unusually sparse area of the universe, then things could look farther away than they really are and there would be no need to rely on dark energy as an explanation for certain astronomical observations.

"If we lived in a very large under-density, then the spacetime itself wouldn't be accelerating," said researcher Timoth: Clifton of Oxford University in England. "It would just be that the observations, if interpreted in the usual way, would look like they were."

Scientists first detected the acceleration by noting that distant supernovae seemed to be moving away from us faster than they should be. 
According to them...

Center $\approx 15 \mathrm{MPC}$

$\approx 50$ Million Light Years

$\approx$ Distance between clusters of galaxies

₹ 1/200 Distance Across

Visible Universe 
According to them...

Extent $\approx 800 \mathrm{MPC}$

$\approx 2.5$ Billion Light Years

$\approx$ 1/5 Distance Across

Visible Universe 


\section{Our view...}

"Modeling an under-density during the $p=0$ stage can only model evolution after the wave has formed, but cannot give an explanation for the creation of such a wave..." $p=0$ is "non-interacting" 


\section{Conclude:}

We are exploring the possibility that these expanding waves might provide a quantitative explanation for the formation of such an underdensity... 


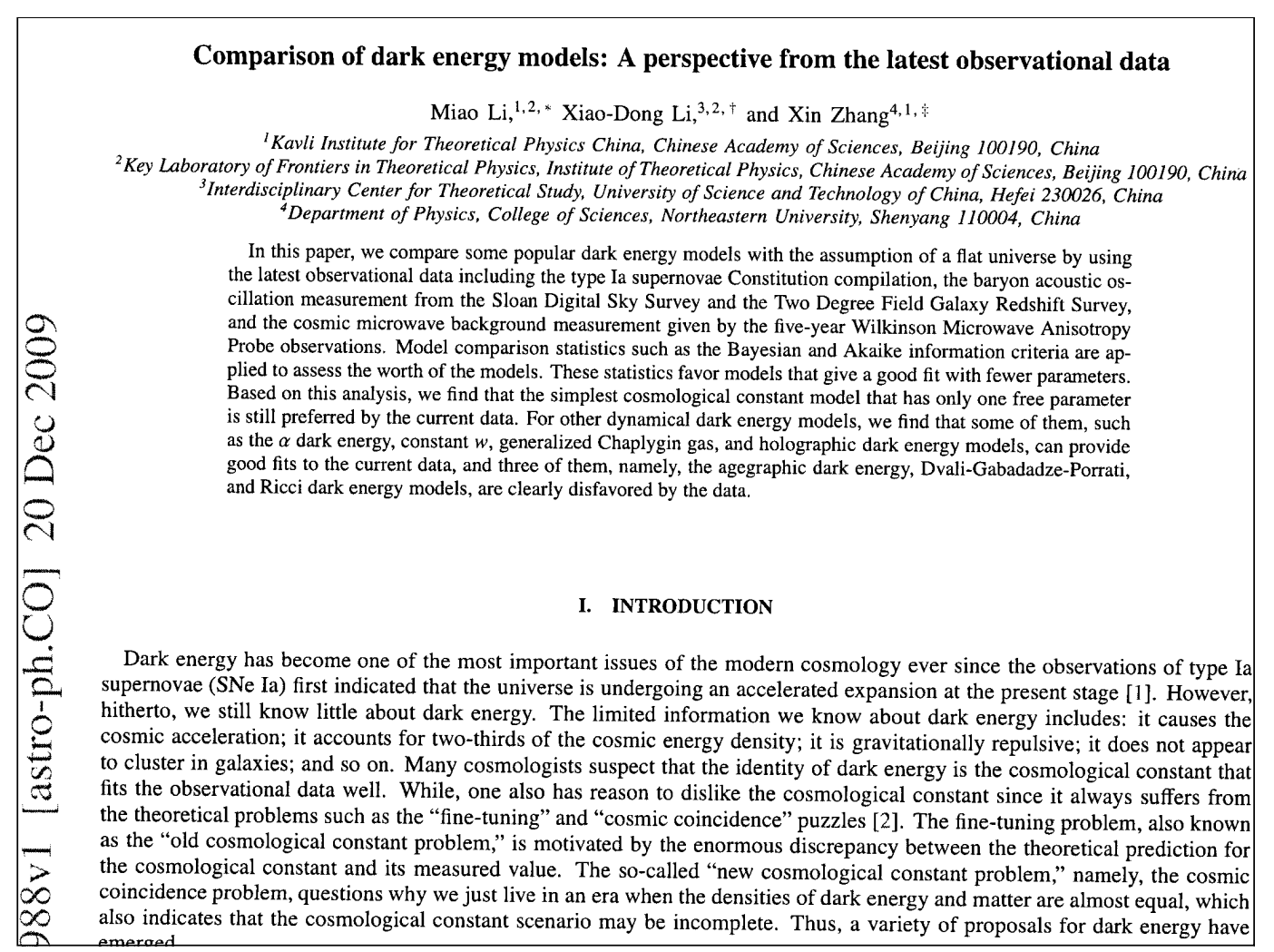




\section{INTRODUCTION}

Dark energy has become one of the most important issues of the modern cosmology ever since the observations of type Ia supernovae (SNe Ia) first indicated that the universe is undergoing an accelerated expansion at the present stage [1]. However, hitherto, we still know little about dark energy. The limited information we know about dark energy includes: it causes the cosmic acceleration; it accounts for two-thirds of the cosmic energy density; it is gravitationally repulsive; it does not appear to cluster in galaxies; and so on. Many cosmologists suspect that the identity of dark energy is the cosmological constant that fits the observational data well. While, one also has reason to dislike the cosmological constant since it always suffers from the theoretical problems such as the "fine-tuning" and "cosmic coincidence" puzzles [2]. The fine-tuning problem, also known

the "old cos
he cosmological constant and its measured value. The so-called "new cosmological constant problem," namely, the cosmic coincidence problem, questions why we just live in an era when the densities of dark energy and matter are almost equal, which also indicates that the cosmological constant scenario may be incomplete. Thus, a variety of proposals for dark energy have emerged.

The possibility that dark energy is dynamical, for example, in a form of some light scalar field [3], has been explored by cosmologists for a long time. A basic way to explore such a dynamical dark energy model in light of observational data is to parameterize dark energy by an equation-of-state parameter $w$, relating the dark energy pressure $p$ to its density $\rho$ via $p=w \rho$. In general, this parameter $w$ is time variable. The most commonly used forms of $w(a)$ involve the constant equation of state, $w=$ const., and the Chevalliear-Polarski-Linder form [4], $w(a)=w_{0}+(1-a) w_{a}$, where $w_{0}$ and $w_{a}$ parameterize the present-day value of $w$ and the first derivative. There are also many other dynamical dark energy models which stem from different aspects of new physics. For example, the "holographic dark energy" models $[5,6,7,8,9,10,11]$ arise from the holographic principle of quantum gravity theory, and the Chaplygin gas models $[12,13,14]$ are motivated by brane world scenarios and may be able to unify dark matter and dark energy. In addition, there is also significant interest in modifications to general relatity in context of explaining the acceleration of the universe. The Dvali-Gabatade-Porrati models $[15,16,17]$ arse from brane-related theories in which gravity leaks out into the bulk at large distances, leading to the accelerated expansion of universe.

In the face of so many competing dark energy candidates, it is important to find an effective way to decide which one is right,

or at least, which one is most favored by the observational data. Although the accumulation of the current observational data has opened a robust window for constraining the parameter space of dark energy models, the model filtration is still a difficult mision own the nergy models. In this paper, we make an effort to assess some popular dark energy models in light of the latest observational 
Dark energy, gravitation and the Copernican principle

Jean-Philippe Uzan

Institut d'Astrophysique de Paris, CNRS-UMR 7095,

Université Pierre \& Marie Curie - Paris VI, 98 bis, Bd Arago, 75014 Paris, France.

(15th August 2008)

To appear in Dark Energy: Ob.servational and Theoretical Approaches, Ed. P. Ruiz-Lapuente (Cambridge University Press, 2010). 

prove that it is the "correct" model of the Universe, in the sense that it is the correct cosmological extrapolation and solution of the local physical laws.

Dark energy confronts us with a compatibility problem since, in order to "save the phenomena" of the observations, we have to include new ingredients (constant, matter fields or interactions) beyond those of our established physical theories. However the recuired value for the simplest dark energy model, i.e. the cosmological constant, is more than 60 order of magnitude smaller to what is expected from theoretical grounds ( $\$ 1.1 .6)$. This tension between what is required by astronomy and what is expected from physics reminds us of the twenty centuries long debate between Aristotelians and Ptolemeans (Duhem, 1913), that was resolved not only by the Copernican model but more important by a better understanding of the physics since 


\section{References:}

\section{- The locally inertial Glimm Scheme...}

- A shock-wave formulation of the Einstein equations, with J. Groah, Meth. and Appl. of Anal., 7, No. 4,(2000), pp. 793-812.

- Shock-wave solutions of the Einstein equations: Existence and consistency by a locally inertial Glimm Scheme, with J. Groah, Memoirs of the AMS, Vol. 172, No. 813, November 2004.

O Shock Wave Interactions in General Relativity: A Locally Inertial Glimm Scheme for Spherically Symmetric Spacetimes, with J. Groah and J. Smoller,

Springer Monographs in Mathematics, 2007. 


\section{References}

- Exact solution incorporating a shock-wave into the standard FRW metric for cosmology...

- Smoller-Temple, Shock-Wave Cosmology Inside a Black Hole, PNAS Sept 2003.

- Smoller-Temple, Cosmology, Black Holes, and Shock Waves Beyond the Hubble Length, Meth. Appl. Anal., 2004. 


\section{References:}

- Connecting the shock wave cosmology model with Guth's theory of inflation...

- How inflationary spacetimes might evolve into spacetimes of finite total mass, with J. Smoller, Meth. and Appl. of Anal., Vol. 12, No. 4, pp. 451-464 (2005).

- How inflation is used to solve the flatness problem, with J. Smoller, Jour. of Hyp. Diff. Eqns. (JHDE) Vol. 3, no. 2, 375-386 (2006). 


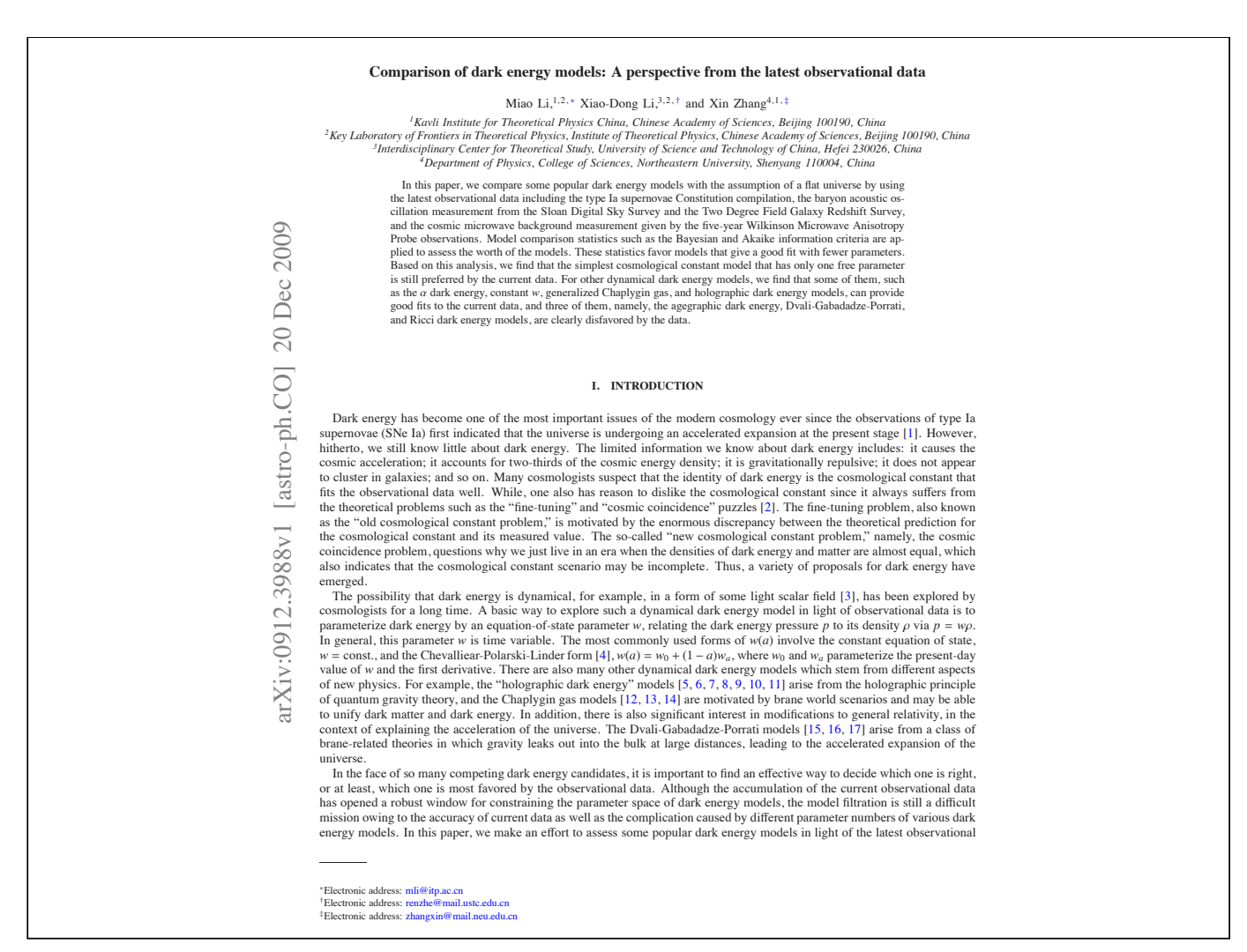




\section{Comparison of dark energy models: A perspective from the latest observational data}

Miao Li, ${ }^{1,2, *}$ Xiao-Dong $\mathrm{Li}^{3,2, \dagger}$ and Xin Zhang ${ }^{4,1,+}$

${ }^{1}$ Kavli Institute for Theoretical Physics China, Chinese Academy of Sciences, Beijing 100190, China

Laboratory of Frontiers in Theoretical Physics, Institute of Theoretical Physics, Chinese Academy of Sciences, Beijing 100190 ${ }^{3}$ Interdisciplinary Center for Theoretical Study, University of Science and Technology of China, Hefei 230026, China

${ }^{4}$ Department of Physics, College of Sciences, Northeastern University, Shenyang 110004, China

In this paper, we compare some popular dark energy models with the assumption of a flat universe by using the latest observational data including the type Ia supernovae Constitution compilation, the baryon acoustic oscillation measurement from the Sloan Digital Sky Survey and the Two Degree Field Galaxy Redshift Survey, and the cosmic microwave background measurement given by the five-year Wilkinson Microwave Anisotropy Probe observations. Model comparison statistics such as the Bayesian and Akaike information criteria are applied to assess the worth of the models. These statistics favor models that give a good fit with fewer parameters. Based on this analysis, we find that the simplest cosmological constant model that has only one free parameter is still pros is still preferred by the current data. For other dynamical dark energy models, we find that some of them, such as the $\alpha$ dark energy, constant $w$, generalized Chaplygin gas, and holographic dark energy models, can provide good fits to the current data, and three of them, namely, the agegraphic dark energy, Dvali-Gabadadze-Porrati, and Ricci dark energy models, are clearly disfavored by the data.

\section{INTRODUCTION}

rk energy has become one of the most important issues of the modern cosmology ever since the observations of novae (SNe Ia) first indicated that the universe is undergoing an accelerated expansion at the present stage [1]. H

to, we still know little about dark energy. The limited information we know about dark energy includes: it cau c acceleration; it accounts for two-thirds of the cosmic energy density; it is gravitationally repulsive; it does not ster in galaxies; and so on. Many cosmologists suspect that the identity of dark energy is the cosmological const 


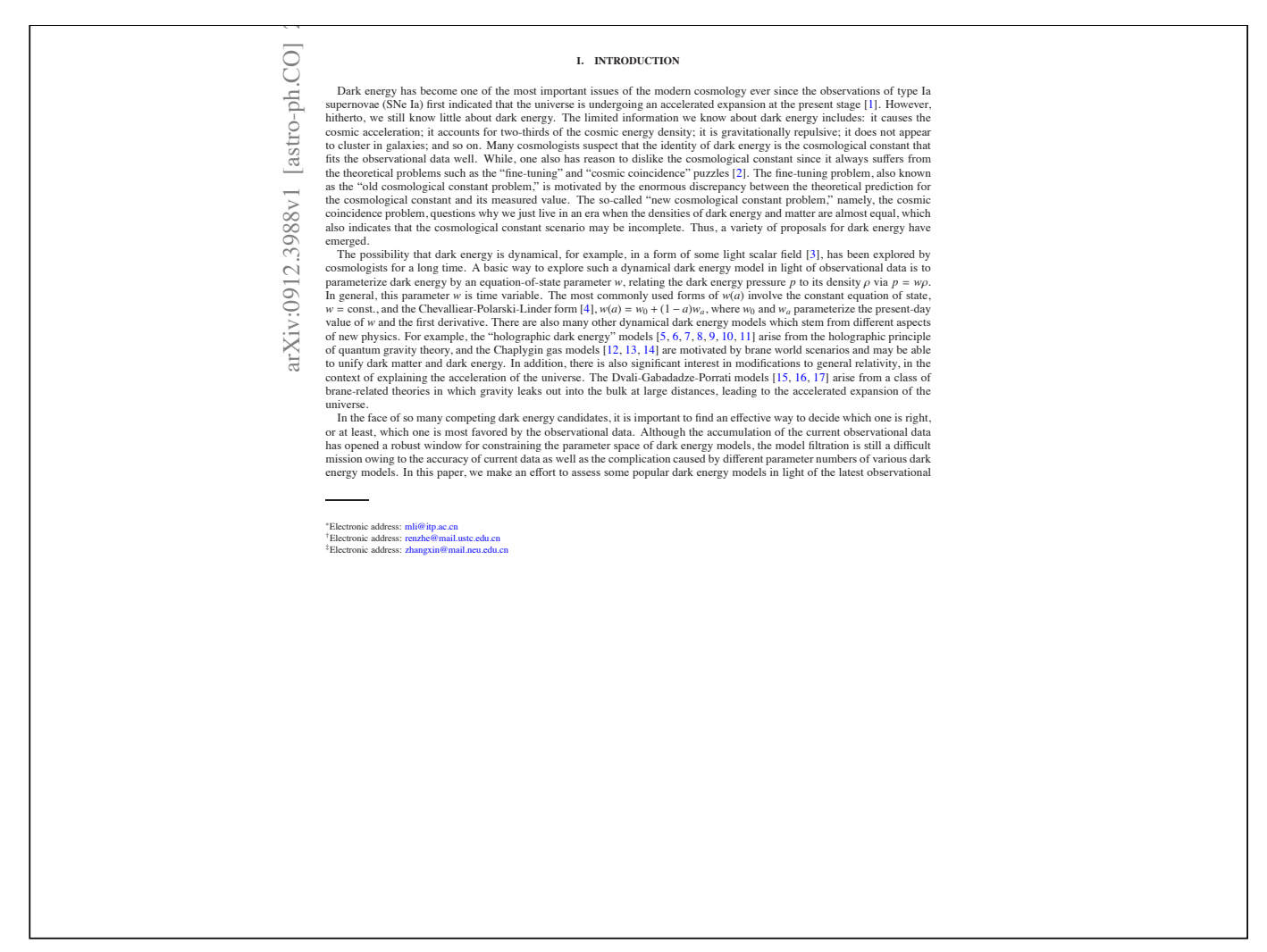




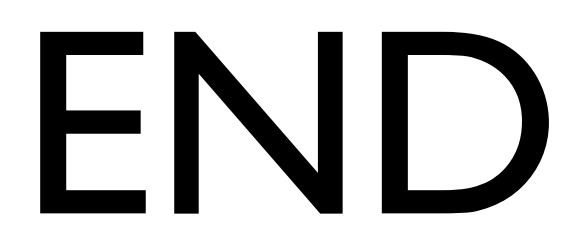




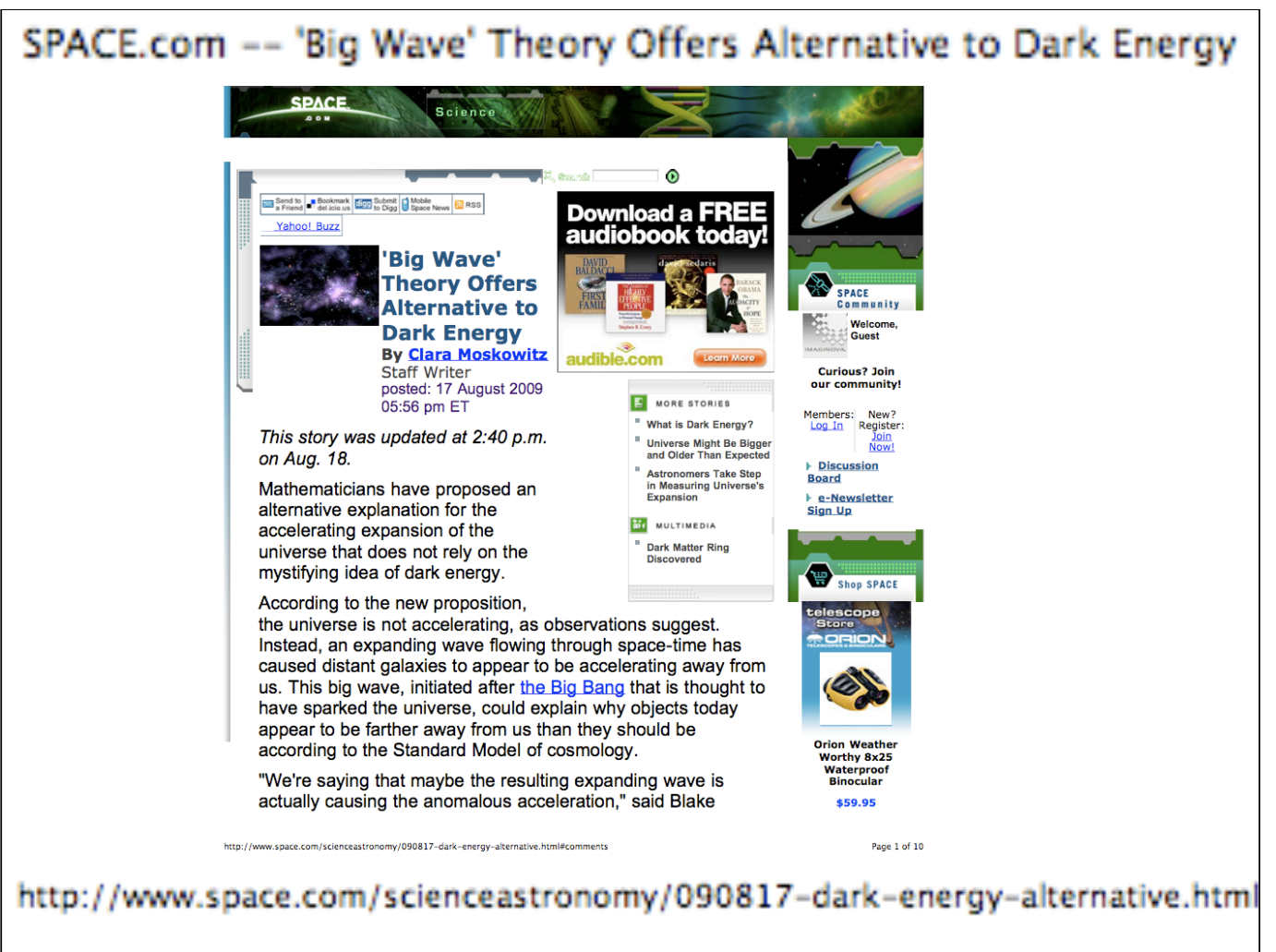




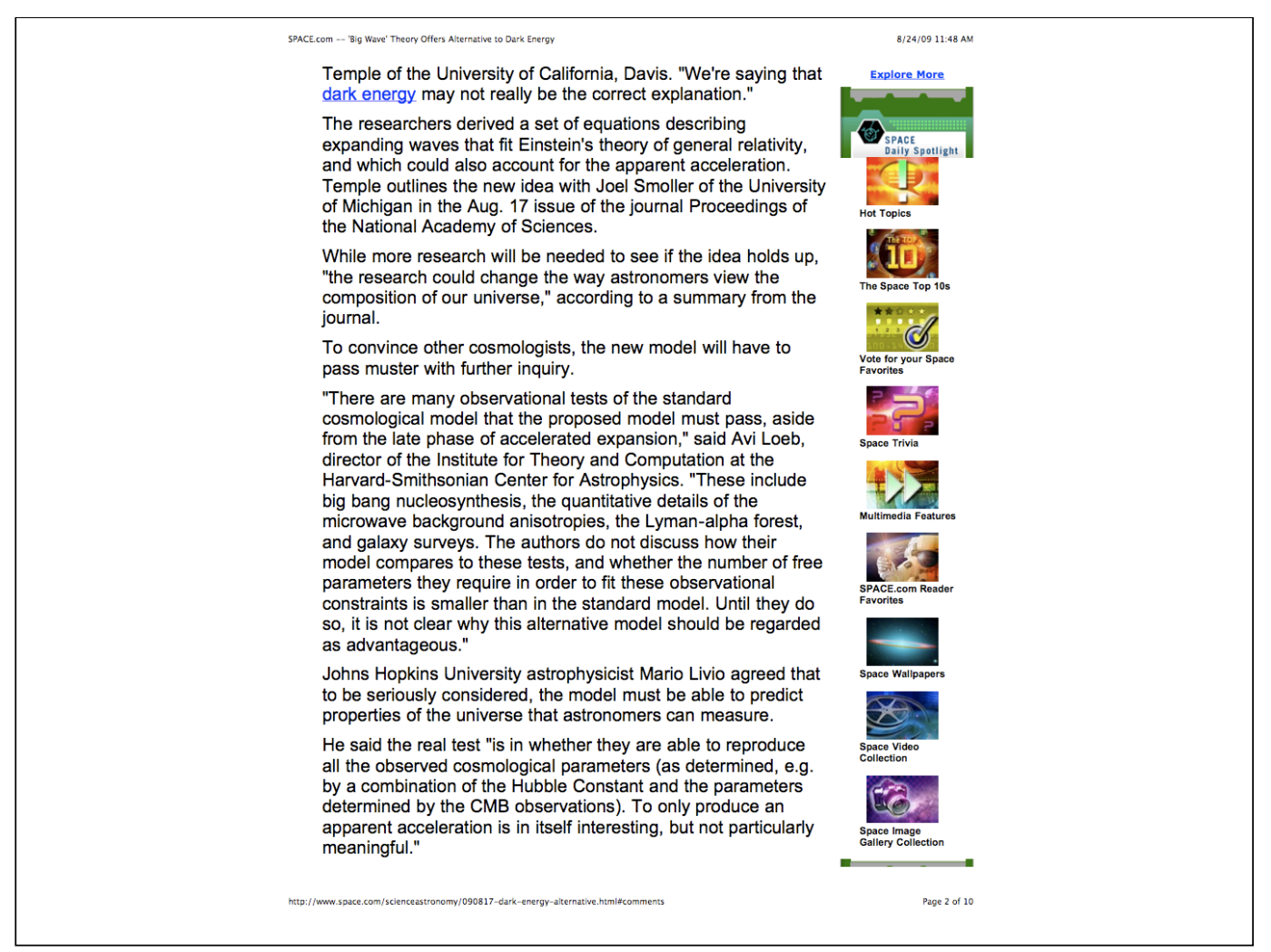


Dark energy is itself a hasty fix to an inconvenient truth

discovered iny astronomers in the late 1990s: that the univer

is expanding, and the rate of this expansion seems to b

To explain this startling finding, cosmologists invoked dark

universe apart in all directions (note that ds puling

separate from the equally mysterious concept of dark matter - a

hypothetical form of matter that populates the universe,

interacting gravitationally with normal matter, but which canno
be seen with light). In this interpretation, the whole universe is

blowing up like a balloon, and from any given point within it, all

distant objects appear to be speeding away from you.

But not everyone is happy with the dark energy explanation.

"It just seems like an unnatural correction to the equations - it's

like a fudge factor," Temple told SPACE.com. "The equations

don't make quite as much physical sense when you put it in.

Temple and Smoller think the idea of an expanding wave

makes more sense.

"At this stage we think this a very plausible theory," Temple
said. "We're saying there isn't any acceleration. The galaxies

are displaced from where they're supposed to be because we're ave that put those galaxies in a slightly

Ripples in a pond

Temple compared the wave to what happens when you throw a

rock into a pond. In this case, the rock would be the Big Bang,

throughout the universe. Later on, when the first galaxies start

to form, they are forming inside space-time that has already

wave. So when we observe those havies with telout the

they don't appear to be where we would expect if there had never been a big wave. 


\section{SPACE.com -- 'Big Wave' Theory Offers Alternative to Dark Ener}

One potential issue with this idea is that it might require a big coincidence.

For the universe to appear to be accelerating at the same rate in all directions, we in the Milky Way would have to be near a local center, at the spot where an expansion wave was initiated local center, at the spot where an expansion wave was
early in the Big Bang when the universe was filled with radiation.

Temple concedes that this is a coincidence, but said it's possible that we are merely in the center of a smaller wave that affects the galaxies we can see from our vantage point - we need not be in the center of the entire universe for the idea to work.

http://www.space.com/scienceastronomy/090817-dark-energy-alternative.html\#comm 


\begin{tabular}{|c|}
\hline $\begin{array}{l}\text { Big Brain Theory: Have Cosmologists Lost Theirs? - New York Times } \\
\text { ÊHe Aicu H Hork Times }\end{array}$ \\
\hline January 15, 2008 \\
\hline Big Brain Theory: Have Cosmologists Lost Theirs? \\
\hline BY DENNIS OVERBYE \\
\hline Correction Appended \\
\hline $\begin{array}{l}\text { It could be the weirdest and most embarrassing prediction in the history of cosmology, if } \\
\text { not science. }\end{array}$ \\
\hline $\begin{array}{l}\text { If true, it would mean that you yourself reading this article are more likely to be some } \\
\text { momentary fluctuation in a field of matter and energy out in space than a person with a } \\
\text { real past born through billions of years of evolution in an orderly star-spangled cosmos. } \\
\text { Your memories and the world you think you see around you are illusions. }\end{array}$ \\
\hline $\begin{array}{l}\text { This bizarre picture is the outcome of a recent series of calculations that take some of } \\
\text { the bedrock theories and discoveries of modern cosmology to the limit. Nobody in the } \\
\text { field believes that this is the way things really work, however. And so in the last couple } \\
\text { of years there has been a growing stream of debate and dueling papers, replete with } \\
\text { references to such esoteric subjects as reincarnation, multiple universes and even the } \\
\text { death of spacetime, as cosmologists try to square the predictions of their cherished } \\
\text { theories with their convictions that we and the universe are real. The basic problem is } \\
\text { that across the eons of time, the standard theories suggest, the universe can recur over }\end{array}$ \\
\hline
\end{tabular}


This bizarre picture is the outcome of a recent series of calculations that take some of the bedrock theories and discoveries of modern cosmology to the limit. Nobody in the field believes that this is the way things really work, however. And so in the last couple of years there has been a growing stream of debate and dueling papers, replete with references to such esoteric subjects as reincarnation, multiple universes and even the death of spacetime, as cosmologists try to square the predictions of their cherished theories with their convictions that we and the universe are real. The basic problem is that across the eons of time, the standard theories suggest, the universe can recur over and over again in an endless cycle of big bangs, but it's hard for nature to make a whole universe. It's much easier to make fragments of one, like planets, yourself maybe in a spacesuit or even - in the most absurd and troubling example - a naked brain floating in space. Nature tends to do what is easiest, from the standpoint of energy and probability. And so these fragments - in particular the brains - would appear far more frequently than real full-fledged universes, or than us. Or they might be us.

Alan Guth, a cosmologist at the Massachusetts Institute of Technology who agrees this overabundance is absurd, pointed out that some calculations result in an infinite number of free-floating brains for every normal brain, making it "infinitely unlikely for us to be normal brains." Welcome to what physicists call the Boltzmann brain problem, named after the 19th-century Austrian physicist Ludwig Boltzmann, who suggested the mechanism by which such fluctuations could happen in a gas or in the universe. 


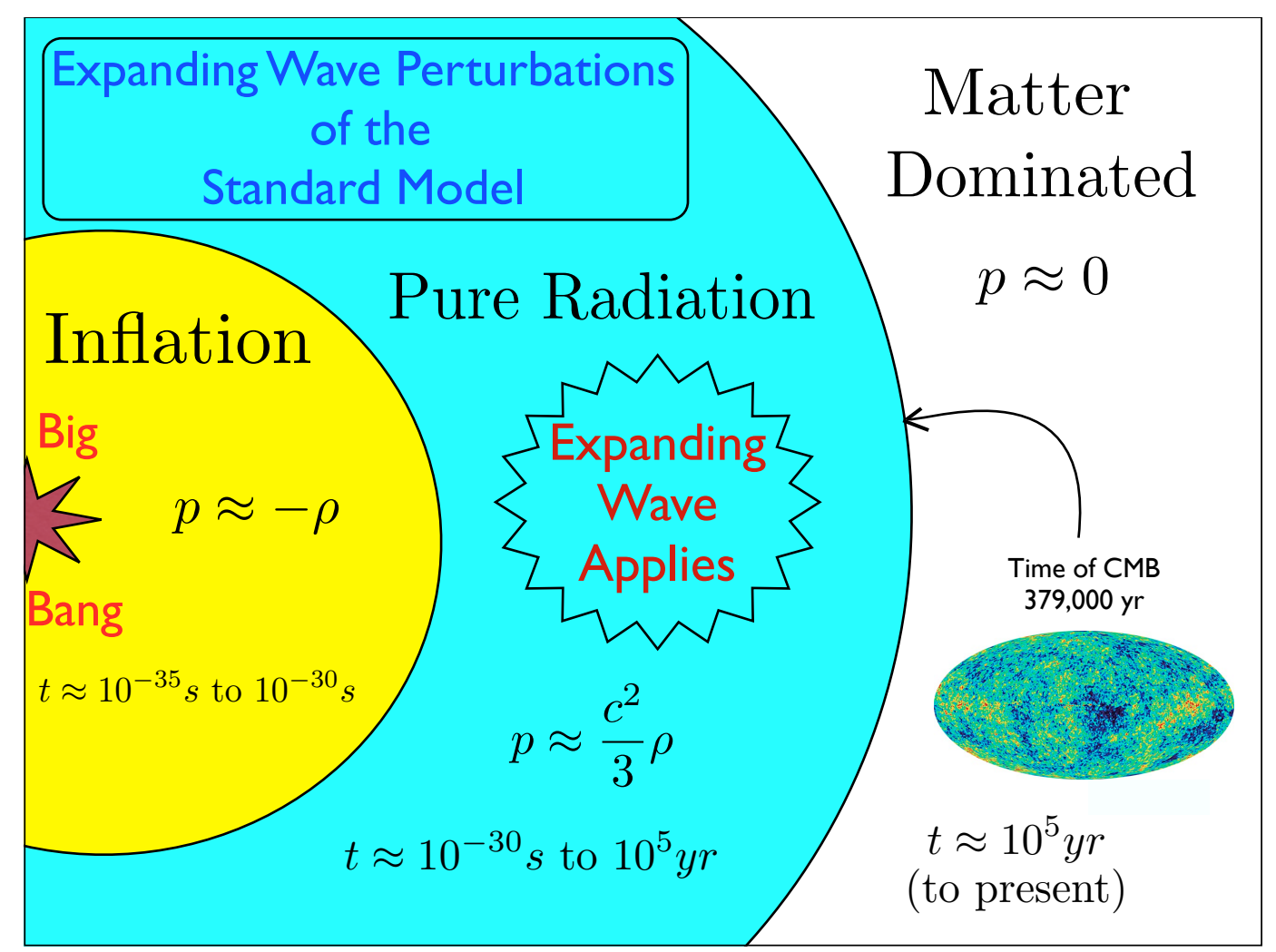




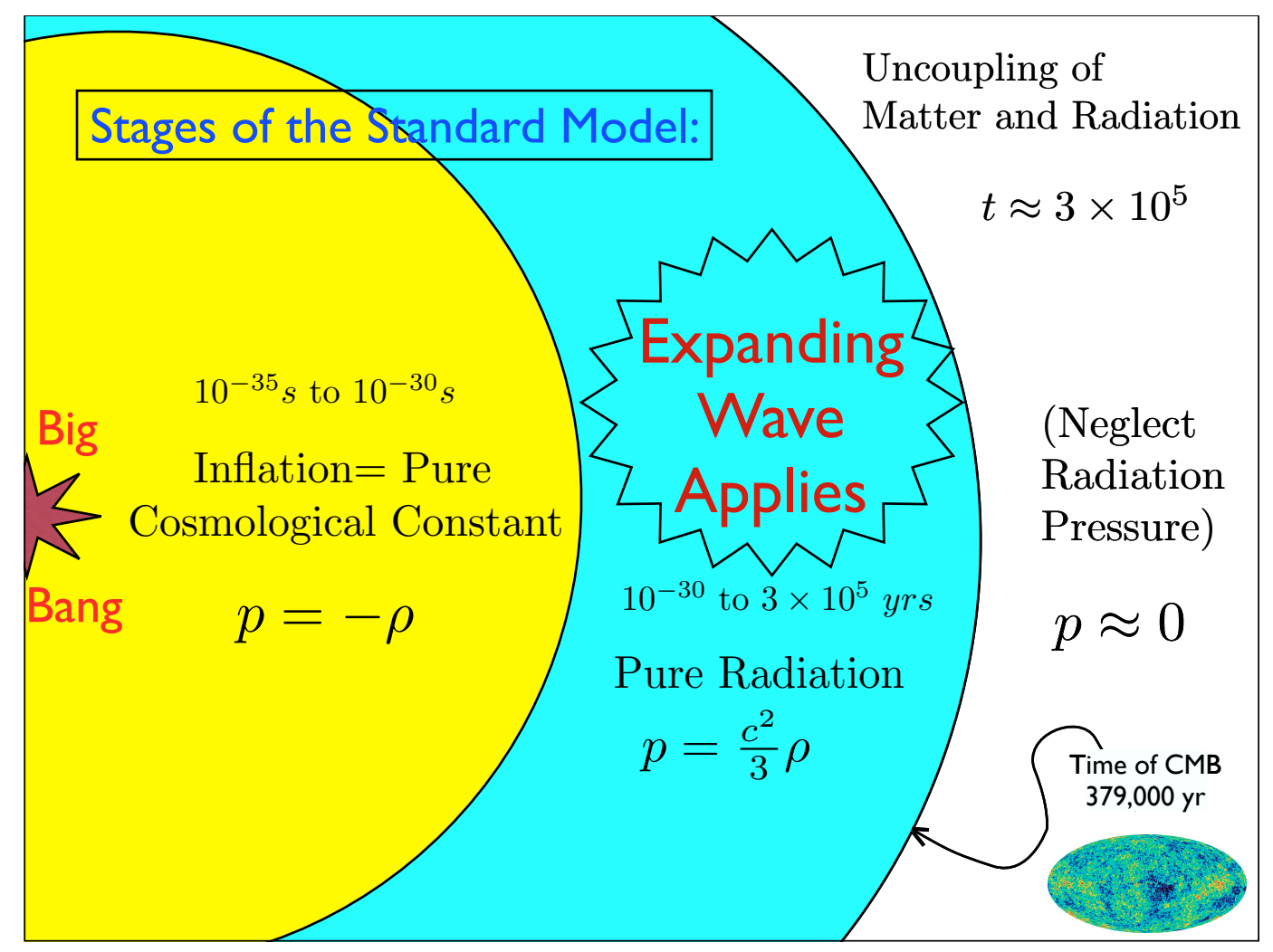

